\title{
The Novel Lie-Algebraic Approach to Studying Integrable Heavenly Type Multi-Dimensional Dynamical Systems
}

\section{Blackmore D ${ }^{1}$, Hentosh $\mathrm{EO}^{2}$ and Prykarpatski $\mathrm{AK}^{3 *}$}

${ }^{1}$ Department of Mathematical Sciences, NJIT, University Heights, Newark, NJ 07102, USA

${ }^{2}$ The Institute for Applied Problems of Mechanics and Mathematics, NAS, Lviv, 79060, Ukraine

${ }^{3}$ Department of Applied Mathematics, AGH University of Science and Technology, Krakow, Poland

\begin{abstract}
The review is devoted to a novel Lie-algebraic approach to studying integrable heavenly type multi-dimensional dynamical systems and its relationships to old and recent investigations of the classical Buhl problem of describing compatible linear vector field equations, its general Pfeiffer and modern Lax-Sato type special solutions. Eespecially we analyze the related Lie-algebra structures and integrability properties of a very interesting class of nonlinear dynamical systems called the dispersionless heavenly type equations, which were initiated by Pleban'ski and later analyzed in a series of articles. The AKS-algebraic and related $\mathcal{R}$-structure schemes are used to study the orbits of the corresponding co-adjoint actions, which are intimately related to the classical Lie-Poisson structures on them. It is demonstrated that their compatibility condition coincides with the corresponding heavenly type equations under consideration. It is shown that all these equations originate in this way and can be represented as a Lax compatibility condition for specially constructed loop vector fields on the torus. The infinite hierarchy of conservations laws related to the heavenly equations is described, and its analytical structure connected with the Casimir invariants, is mentioned. In addition, typical examples of such equations, demonstrating in detail their integrability via the scheme devised herein, are presented. The relationship of a very interesting Lagrange-d'Alembert type mechanical interpretation of the devised integrability scheme with the Lax-Sato equations is also discussed.
\end{abstract}

Keywords: Lax-Sato equations; Heavenly equations; Lax integrability; Hamiltonian system; Torus diffeomorphisms; Loop Lie algebra; Liealgebraic scheme; Casimir invariants; R-structure; Lie-Poisson structure; Bi-Hamiltonicity; Lagrange-d'Alembert principle

\section{Introduction}

In 1928 the French mathematician Buhl in his works [1,2] posed the problem of classifying all infinitesimal symmetries of a given linear vector field equation

\section{$A \psi=0$}

where function $\psi \in C^{2}\left(\mathbb{R}^{n} ; \mathbb{R}\right)$, and

$$
A:=\sum_{j=1, n} a_{j}(x) \frac{\partial}{\partial x_{j}}
$$

is a vector field operator on $\mathbb{R}^{n}$ with coefficients $a_{j} \in C^{1}\left(\mathbb{R}^{n} ; \mathbb{R}\right), j=\overline{1, n}$. It is easy to show that the problem under regard is reduced [3] to describing all possible vector fields

$$
A^{(k)}:=\sum_{j=1, n} a_{j}^{(k)}(x) \frac{\partial}{\partial x_{j}}
$$

with coefficients $a_{j}^{(k)} \in C^{1}\left(\mathbb{R}^{n} ; \mathbb{R}\right), j, k=\overline{1, n}$, satisfying the Lax type commutator condition

$$
\left.A, A^{(k)}\right]=0
$$

for all $x \in \mathbb{R}^{n}$ and $k=\overline{1, n}$. This M.A. Buhl problem above was completely solved in 1931 by the Ukrainian mathematician Pfeiffer in the works [4-8]; where he has constructed explicitly the searched set of independent vector fields (3); having made use effectively of the full set of invariants for the vector field (2) and the related solution set structure of the Jacobi-Mayer system of equations; naturally following from (4). Some results; yet not complete; were also obtained by Popovici [9].

Some years ago the Buhl MA type equivalent problem was independently reanalyzed once more by Japanese mathematicians Takasaki and Takebe $[10,11]$ and later by Bogdanov et al. [12] for a very special case when the vector field operator (2) depends analytically on a "spectral" parameter $\lambda \in \mathbb{C}$ :

$$
\tilde{A}:=\frac{\partial}{\partial t}+\sum_{j=1, n} a_{j}(t, x ; \lambda) \frac{\partial}{\partial x_{j}}+a_{0}(t, x ; \lambda) \frac{\partial}{\partial \lambda} .
$$

Based on the before developed Sato theory $[13,14]$; the authors mentioned above have shown for some special kinds of vector fields (5) that there exists an infinite hierarchy of the symmetry vector fields

$$
\tilde{A}^{(k)}:=\frac{\partial}{\partial \tau_{k}}+\sum_{j=1, n} a_{j}^{(k)}(\tau, x ; \lambda) \frac{\partial}{\partial x_{j}}+a_{0}^{(k)}(\tau, x ; \lambda) \frac{\partial}{\partial \lambda},
$$

where $\tau=\left(t ; \tau_{1}, \tau_{2}, \ldots\right) \in \mathbb{R}^{\mathbb{Z}_{+}}, k \in \mathbb{Z}_{+}$, satisfying the Lax-Sato type compatible commutator conditions

$$
\left.\tilde{A}, \tilde{A}^{(k)}\right]=0=\left[\tilde{A}^{(j)}, \tilde{A}^{(k)}\right]
$$

for all $k, j \in \mathbb{Z}_{+}$. Moreover; in the cases under regard; the compatibility conditions (7) proved to be equivalent to some very important for applications heavenly type dispersionless equations in partial derivatives.

In the present work we investigate the Lax-Sato compatible systems; the related Lie-algebraic structures and complete integrability

*Corresponding author: Prykarpatski AK, Department of Applied Mathematics, AGH University of Science and Technology, Krakow, Poland, Tel: +48-535-531 185; E-mail: pryk.anat@cybergal.com

Received October 07, 2017; Accepted December 15, 2017; Published December 22, 2017

Citation: Blackmore D, Hentosh EO, Prykarpatski AK (2017) The Novel LieAlgebraic Approach to Studying Integrable Heavenly Type Multi-Dimensional Dynamical Systems. J Generalized Lie Theory Appl 11: 286. doi: 10.4172/17364337.1000286

Copyright: (c) 2017 Blackmore D, et al. This is an open-access article distributed under the terms of the Creative Commons Attribution License, which permits unrestricted use, distribution, and reproduction in any medium, provided the original author and source are credited. 
properties of an interesting class of nonlinear dynamical systems called the heavenly type equations; which were introduced by Pleban'ski [15] and analyzed in a series of articles [10-12,16-20]. In our work; having employed the AKS-algebraic and related $\mathcal{R}$-structure schemes [21-26]; applied to the holomorphic loop Lie algebra $\tilde{\mathcal{G}}:=\widetilde{\operatorname{diff}}\left(\mathbb{T}^{n}\right)$ of vector fields on torus $\mathbb{T}^{n}, n \mathbb{Z}$, the orbits of the corresponding coadjoint actions on $\tilde{\mathcal{G}}^{*}$; closely related to the classical Lie-Poisson type structures; were reanalyzed and studied in detail. By constructing two commuting flows on the coadjoint space $\tilde{\mathcal{G}}^{*}$, generated by a chosen root element $\tilde{l} \in \tilde{\mathcal{G}}^{*}$ and some Casimir invariants; we have successively demonstrated that their compatibility condition coincides exactly with the corresponding heavenly equations under consideration.

As a by-product of the construction; devised recently in works $[27,28]$; we prove that all the heavenly equations have a similar origin and can be represented as a Lax compatibility condition for special loop vector fields on the torus $\mathbb{T}^{n}$. We analyze the structure of the infinite hierarchy of conservations laws; related to the heavenly equations; and demonstrate their analytical structure connected with the Casimir invariants is generated by the Lie-Poisson structure on $\tilde{\mathcal{G}}^{*}$. Moreover; we have extended the initial Lie-algebraic structure for the case when the the basic Lie algebra $\tilde{\mathcal{G}} \widetilde{\operatorname{diff}}\left(\mathbb{T}^{n}\right)$ is replaced by the adjacent holomorphic Lie algebra $\overline{\mathcal{G}}:=\operatorname{diff}_{\text {hol }}\left(\mathbb{C} \times \mathbb{T}^{n}\right) \subset \operatorname{diff}\left(\mathbb{C} \times \mathbb{T}^{n}\right)$ of vector fields on $\mathbb{C} \times \mathbb{T}^{n}$. Typical examples are presented for all cases of the heavenly equations and it is shown in detail and their integrability is demonstrated using the scheme devised here. This scheme makes it possible to construct a very natural derivation of well known Lax-Sato representation for an infinite hierarchy of heavenly equations; related to the canonical Lie-Poisson structure on the adjoint space $\overline{\mathcal{G}}^{*}$. We also briefly discuss the Lagrangian representation of these equations following from their Hamiltonicity with respect to both intimately related commuting evolutionary flows; and the related bi-Hamiltonian structure as well as the Bäcklund transformations. As a matter of fact; there are only a few examples of multi-dimensional integrable systems for which such a detailed description of their mathematical structure has been given. As was aptly mentioned [29]; the heavenly equations comprise an important class of such integrable systems. This is due in part to the fact that some of them are obtained by a reduction of the Einstein equations with Euclidean (and neutral) signature for (anti-) self-dual gravity; which includes the theory of gravitational instantons. This and other cases of important applications of multi-dimensional integrable equations strongly motivated us to study this class of equations and the related mathematical structures. As a very interesting aspect of our approach to describing integrability of the heavenly dynamical systems; there is a very interesting Lagrange-d'Alembert type mechanical interpretation. We need to underline here that the main motivating idea behind this work was based both on the paper by Kulish [30]; devoted to studying the super-conformal Korteweg-de-Vries equation as an integrable Hamiltonian flow on the adjoint space to the holomorphic loop Lie superalgebra of super-conformal vector fields on the circle; and on the insightful investigation by Mikhalev [31]; which studied Hamiltonian structures on the adjoint space to the holomorphic loop Lie algebra of smooth vector fields on the circle. We were also impressed by deep technical results $[10,11]$ of Takasaki and Takebe; who fully realized the vector field scheme of the Lax-Sato theory. Additionally; we were strongly influenced both by the works of Pavlov; Bogdanov; Dryuma; Konopelchenko and Manakov [12,32-34]; as well as by the work of Ferapontov and Moss [35]; in which they devised new effective differential-geometric and analytical methods for studying an integrable degenerate multi-dimensional dispersionless heavenly type hierarchy of equations; the mathematical importance of which is still far from being properly appreciated. Concerning other Lie-algebraic approaches to constructing integrable heavenly equations; we mention work by Szablikowski and Sergyeyev [36,37]; Ovsienko $[17,18]$ and by Kruglikov and Morozov [38].

We present interesting examples of the Lie-algebraic description of typical integrable heavenly equations amongst which the MikhalevPavlov equation [31]; the first and second reduced Shabat type [39] and Hirota heavenly equations [40], the Liouville type [33] equations and some other.

We also generalized the Lie-algebraic scheme $[27,28]$ subject to the loop Lie algebra $\widetilde{\operatorname{diff}}\left(\mathbb{S}^{1 \mid N}\right)$ of superconformal vector fields on $\mathbb{S}^{1 \mid N}$, being a Lie algebra of the Lie group of superconformal diffeomorphisms of the $1 \mid N$-dimensional supertorus $\mathbb{S}^{1 / N} \simeq \mathbb{S}^{1} \times \Lambda_{1}^{N}$, where $\Lambda:=\Lambda_{0} \oplus \Lambda_{1}$ is an infinite-dimensional Grassmann algebra over $\mathbb{C}, \Lambda_{0} \supset \mathbb{C}$. It is applied to constructing the Lax-Sato integrable superanalogs of the MikhalevPavlov type heavenly super-equation for every $N \in \mathbb{N} \backslash\{4 ; 5\}$. As a result of suitably chosen superconformal mappings in the space of variables $\left(z ; \theta_{1}, \ldots, \theta_{N}\right) \in \mathbb{S}^{1 \mid N}$ the superanalogs of Liouville type equations are obtained by means of using the loop Lie superalgebra $\widetilde{\operatorname{diff}}\left(\mathbb{S}^{1 / N}\right)$. Some results are also presented for a special Lie-algebraic integrability scheme based on a metrized loop Lie algebra; generated by a semisimple sum of the holomorphic Lie algebra $\overline{\mathcal{G}}=\operatorname{diff}_{\text {hol }}\left(\mathbb{C} \times \mathbb{T}^{n}\right)$ and its coadjoint space $\overline{\mathcal{G}}^{*}$.

\section{The Lax-Sato Type Compatible Systems of Linear Vector Field Equations}

\section{A vector field on the torus and its invariants}

Consider a simple vector field $X: \mathbb{R}^{n} T\left(\mathbb{R} \times \mathbb{T}^{n}\right)$ on the $(n+1)$ dimensional toroidal manifold $\mathbb{R}^{n}$ for arbitrary

$n \in \mathbb{Z}_{+}$, which we will write in the slightly special form

$$
A=\frac{\partial}{\partial t}+<a(t, x), \frac{\partial}{\partial x}>,
$$

where $(t, x) \in \mathbb{R} \times \mathbb{T}^{n}, a(t, x) \in \mathbb{E}^{n}, \frac{\partial}{\partial x}:=\left(\frac{\partial}{\partial x_{1}}, \frac{\partial}{\partial x_{2}}, \ldots, \frac{\partial}{\partial x_{n}}\right)^{\top}$ and $\left.<\cdot, \cdot\right\rangle$ is the standard scalar product on the Euclidean space

$\mathbb{E}^{n}$. With the vector field (8); one can associate the linear equation

$$
A \psi=0
$$

for some function $\in C^{2}\left(\mathbb{R T}^{n} ; \mathbb{R}\right)$, which we will call an "invariant" of the vector field.

Next; we study the existence and number of such functionallyindependent invariants to eqn. (9). For this let us pose the following Cauchy problem for eqn. (9): Find a function $\psi \in C^{2}\left(\mathbb{R} \mathbb{T}^{n} ; \mathbb{R}\right)$, which at point $t^{(0)} \in \mathbb{R}$ satisfies the condition $\left.\psi(t, x)\right|_{t=t^{(0)}}=\bar{\psi}(x), \quad x \in \mathbb{R}^{n}$, for a given function $\bar{\psi} \in C^{2}\left(\mathbb{T}^{n} ; \mathbb{R}\right)$. For eqn. (9) there is naturally related parametric vector field on the torus $\mathbb{T}^{n}$ in the form of the ordinary vector differential equation

$$
d x / d t=a(t, x),
$$

to which there corresponds the following Cauchy problem: find a function $x: \mathbb{R} \rightarrow \mathbb{T}^{n}$ satisfying

$$
\left.x(t)\right|_{t=t^{(0)}}=z
$$

for an arbitrary constant vector $z \in \mathbb{T}^{n}$. Assuming that the vectorfunction $a C^{1}\left(\mathbb{R} \times \mathbb{T}^{n} ; \mathbb{R}^{n}\right)$; it follows from the classical Cauchy theorem [41] on the existence and unicity of the solution to (10) and (11); 
Citation: Blackmore D, Hentosh EO, Prykarpatski AK (2017) The Novel Lie-Algebraic Approach to Studying Integrable Heavenly Type MultiDimensional Dynamical Systems. J Generalized Lie Theory Appl 11: 286. doi: 10.4172/1736-4337.1000286

that we can obtain a unique solution to the vector eqn. (10) as some function $\Phi \in C^{1}\left(\mathbb{R} \times \mathbb{T}^{n} ; \mathbb{T}^{n}\right), x=\Phi(t, z)$, such that the matrix $\partial \Phi(t, z) \partial z$ is nondegenerate for all $t \in \mathbb{R}$ sufficiently close to $t^{(0)} \in \mathbb{R}$. Hence; the Implicit Function Theorem [42,43] implies that there exists a mapping $\Psi: \mathbb{R}^{n} \mathbb{T}^{n}$, such that

$$
\Psi(t, x)=z
$$

for every $z \in \mathbb{T}^{n}$ and all $t \in \mathbb{R}$ sufficiently enough to $t^{(0)} \in \mathbb{R}$. Supposing now that the functional vector $\Psi(t, x)=\left(\psi^{(1)}(t, x), \psi^{(2)}(t, x), \ldots, \psi^{(n)}(t, x)\right)^{\top},(t, x) \in \mathbb{R} \times \mathbb{T}^{n}, \quad$ is $\quad$ constructed; from the arbitrariness of the parameter $z \in \mathbb{T}^{n}$ one can deduce that all functions $\psi^{(j)}: \mathbb{R} \times \mathbb{T}^{n} \rightarrow \mathbb{R}, \quad j=\overline{1, n}$, are functionally independent invariants of the vector field eqn. (9); that is $A \psi^{(j)}=0, j=\overline{1, n}$. Thus; the vector field eqn. (9) has exactly $n \in \mathbb{Z}_{+}$functionally independent invariants; which make it possible; in particular; to solve the Cauchy problem posed above. Namely; let a mapping $\alpha: \mathbb{R}^{n} \mathbb{R}$ be chosen such that $\left.\alpha(\Psi(t, x))\right|_{t=t^{(0)}}=\bar{\psi}(x)$ for all $x \in \mathbb{R}^{n}$ and a fixed $t^{(0)} \in \mathbb{R}$. Inasmuch as the superposition of functions $\alpha \circ \Psi: \mathbb{R} \times \mathbb{T}^{n} \rightarrow \mathbb{R}$ is; evidently; also an invariant for the eqn. (9); it provides the solution to this Cauchy problem; which we can formulate as the following classical lemma.

Lemma 2.1: The linear eqn. (9); generated by the vector field (10) on the toroidal manifold $\mathbb{R} \times \mathbb{T}^{n}$, has exactly $n \mathbb{Z}$ functionally independent invariants.

Consider now a differential form $\chi^{(n)} \in \Lambda^{n}\left(\mathbb{T}^{n}\right)$, generated by the vector of independent invariants (12); additionally depending parametrically on the vector evolution parameter $t \mathbb{R}^{n}$ :

$$
\chi^{(n)}:=\mathrm{d} \psi^{(1)} \wedge \mathrm{d} \psi^{(2)} \wedge \ldots \wedge \mathrm{d} \psi^{(n)},
$$

where; by definition; for any $\psi \in C^{2}\left(\mathbb{R}^{n} \times \mathbb{T}^{n} ; \mathbb{R}\right)$ the differential

$$
\mathrm{d} \psi:=<\frac{\partial \psi}{\partial x}, d x>\text {. }
$$

As follows from the Frobenius theorem [35,42,44]; the Plucker type form (13) is for $t \in \mathbb{R}^{n}$ nonzero on the torus $\mathbb{T}^{n}$ owing to the functional independence of the invariants. It is easy to see that the following [9] Jacobi-Mayer type relationship

$$
\left|\frac{\partial \Psi}{\partial x}\right|^{-1} \mathrm{~d} \psi^{(1)} \wedge \mathrm{d} \psi^{(2)} \wedge \ldots \wedge \mathrm{d} \psi^{(n)}=d x_{1} \wedge d x_{2} \wedge \ldots \wedge d x_{n},
$$

holds on the manifold $\mathbb{T}^{n}$ where on the right-hand side one has the volume measure on the torus $\mathbb{T}^{n}$, which is naturally dependent on $t \in \mathbb{R}^{n}$ owing to the vector field relationships (10). Taking into account that for all invariants $\psi^{(k)} \in C^{2}\left(\mathbb{R}^{\mathbb{Z}_{+}} \times \mathbb{T}^{n} ; \mathbb{R}\right) k=\overline{1, n}$ there hold the differential expressions

$$
\mathrm{d} \psi^{(k)}=-\sum_{s=1, n} \frac{\partial \psi^{(k)}}{\partial t_{s}} d t_{s},
$$

their substitution into (15) gives rise; owing to the independence of the differentials $d t_{s}, s=\overline{1, n}$, to the following set of the compatible vector field relationships

$$
\frac{\partial \Psi}{\partial t_{s}}+\sum_{j, k=1, n}\left[\left(\frac{\partial \Psi}{\partial x}\right)_{j k}^{-1} \frac{\partial \psi^{(k)}}{\partial t_{s}}\right] \frac{\partial \Psi}{\partial x_{j}}=0
$$

for any $s=\overline{1, n}$. The latter property; as it was demonstrated by Pfeiffer [9]; makes it possible to solve effectively the M.A. Buhl problem and has interesting applications $[13,35]$ in the theory of completely integrable dynamical systems of heavenly type; which are considered in the next section.

\section{Vector field hierarchies on the torus with "spectral" parameter and the Lax-Sato integrable heavenly dynamical systems}

Consider some naturally ordered infinite set of parametric vector fields (8) on the infinite dimensional toroidal manifold $\mathbb{R}^{\mathbb{Z}_{+}} \times \mathbb{T}^{n}$ in the form

$$
A^{(k)}=\frac{\partial}{\partial t_{k}}+<a^{(k)}(t, x ; \lambda), \frac{\partial}{\partial x}>+a_{0}^{(k)}(t, x ; \lambda) \frac{\partial}{\partial \lambda}:=\frac{\partial}{\partial t_{k}}+\mathrm{A}^{(k)},
$$

where $t_{k} \in \mathbb{R}, k \in \mathbb{Z}_{+},(t, x ; \lambda) \in\left(\mathbb{R}^{\mathbb{Z}_{+}} \times \mathbb{T}^{n}\right) \times \mathbb{C}$ are the evolution parameters; and the dependence of smooth vectors $\left(a_{0}^{(k)}, a^{(k)}\right)^{\top} \in \mathbb{E} \times \mathbb{E}^{n}, k \in \mathbb{Z}_{+}$, on the "spectral" parameter $\lambda=\mathbb{C}$ is assumed to be holomorphic. Suppose now that the infinite hierarchy of linear equations

$$
A^{(k)} \psi=0
$$

for $k \mathbb{Z}_{+}$has exactly $n+1 \in \mathbb{Z}_{+}$common functionally independent invariants $\psi^{(j)}(\lambda) \in C^{2}\left(\mathbb{R}^{\mathbb{Z}_{+}} \times \mathbb{T}^{n} ; \mathbb{C}\right), j=\overline{0, n}$ on the torus $\mathbb{T}^{n}$, suitably depending on the parameter $\lambda \in \mathbb{C}$. Then; owing to the existence theory $[42,43]$ for ordinary differential equations depending on the "spectral" parameter $\lambda \in \mathbb{C}$, these invariants may be assumed to be such that allow analytical continuation in the parameter $\lambda \in \mathbb{C}$ both inside $\mathbb{S}_{+}^{1} \subset \mathbb{C}$ of some circle $\mathbb{S}^{1} \subset \mathbb{C}$ and subject to the parameter $\lambda^{-1} \in \mathbb{C},|\lambda| \rightarrow \infty$, outside $\mathbb{S}_{-}^{1} \subset \mathbb{C}$ of this cirrle $\mathbb{S}^{1} \subset \mathbb{C}$. This means that as $|\lambda| \rightarrow \infty$ we have the following expansions:

$$
\begin{aligned}
& \psi^{(0)}(\lambda) \sim \lambda+\sum_{k=0}^{\infty} \psi_{k}^{(0)}(t, x) \lambda^{-k}, \\
& \psi^{(1)}(\lambda) \sim \sum_{k=0}^{\infty} \tau_{k}^{(1)}(t, x) \psi_{0}(\lambda)^{k}+\sum_{k=1}^{\infty} \psi_{k}^{(1)}(t, x) \psi_{0}(\lambda)^{-k}, \\
& \psi^{(2)}(\lambda) \sim \sum_{k=0}^{\infty} \tau_{k}^{(2)}(t, x) \psi_{0}(\lambda)^{k}+\sum_{k=1}^{\infty} \psi_{k}^{(2)}(t, x) \psi_{0}(\lambda)^{-k}, \\
& \ldots \\
& \psi^{(n)}(\lambda) \sim \sum_{k=0}^{\infty} \tau_{k}^{(n)}(t, x) \psi_{0}(\lambda)^{k}+\sum_{k=1}^{\infty} \psi_{k}^{(n)}(t, x) \psi_{0}(\lambda)^{-k},
\end{aligned}
$$

where we took into account that $\psi^{(0)}(\lambda) \in C^{2}\left(\mathbb{R}^{\mathbb{Z}_{+}} \times \mathbb{T}^{n} ; \mathbb{C}\right), \lambda \in \mathbb{C}$, is the basic invariant solution to eqn. (19); the functions $\tau_{l}^{(s)} \in C^{2}\left(\mathbb{R}^{\mathbb{Z}_{+}} \times \mathbb{T}^{n} ; \mathbb{R}\right)$ for all $s=\overline{1, n}, l \in \mathbb{Z}_{+}$, are assumed to be independent and $\psi_{k}^{(j)} \in C^{2}\left(\mathbb{R}^{\mathbb{Z}_{+}} \times \mathbb{T}^{n} ; \mathbb{R}\right)$ for all $k \in \mathbb{N}, j=\overline{0, n}$, are arbitrary. Write down now the condition (15) on the manifold $\mathbb{C} \times \mathbb{T}^{n}$ in the form

$$
\left|\frac{\partial \Psi}{\partial \mathrm{x}}\right|^{-1} \mathrm{~d} \psi^{(0)} \wedge \mathrm{d} \psi^{(1)} \wedge \mathrm{d} \psi^{(2)} \wedge \ldots \wedge \mathrm{d} \psi^{(n)}=d \lambda \wedge d x_{1} \wedge d x_{2} \wedge \ldots \wedge d x_{n},
$$

where $\mathrm{x}:=(\lambda, x) \in \mathbb{C} \times \mathbb{T}^{n}, \quad\left|\frac{\partial \Psi}{\partial \mathrm{x}}\right|$ is the Jacobi determinant of the mapping $\Psi:=\left(\psi^{(0)}, \psi^{(1)}, \psi^{(2)}, \ldots, \psi^{(n)}\right)^{\top} \in C^{2}\left(\mathbb{C} \times\left(\mathbb{R}^{\mathbb{Z}_{+}} \times \mathbb{T}^{n}\right) ; \mathbb{C}^{n+1}\right)$ on the manifold $\mathbb{C} \times \mathbb{T}^{n}$ Inasmuch this mapping subject to the parameter $\lambda \in \mathbb{C}$ has analytical continuation $[43]$ inside $\mathbb{S}_{+}^{1} \subset \mathbb{C}$ of the circle $\mathbb{S}^{1} \subset \mathbb{C}$ and subject to the parameter $\lambda^{-1} \mathbb{C}$ as $|\lambda| \rightarrow \infty$ outside $\mathbb{S}_{-}^{1} \subset \mathbb{C}$ of this circle $\mathbb{S}^{1} \subset \mathbb{C}$, one can easily obtain from the vanishing differential expressions

$$
d \psi^{(j)}=\mathrm{d} \psi^{(j)}+\sum_{k=0}^{\infty} \frac{\partial \psi^{(j)}}{\partial \tau_{k}^{(j)}} d \tau_{k}^{(j)}=0
$$

for all $j=\overline{1, n}$ and the relationship (21) on the manifold $\mathbb{C T}^{n}$ of the independent variables $x \in \mathbb{C T}^{n}$, evolving analytically with respect to the parameters $\tau_{k}^{(j)} \in \mathbb{R}, \quad j=\overline{1, n}, k \in \mathbb{Z}_{+}$, the following Lax-Sato criterion:

$$
\left(\left|\frac{\partial \Psi}{\partial \mathrm{x}}\right|^{-1} \mathrm{~d} \psi^{(0)} \wedge \mathrm{d} \psi^{(1)} \wedge \mathrm{d} \psi^{(2)} \wedge \ldots \wedge \mathrm{d} \psi^{(n)}\right)_{-}=0,
$$

where (...)_ means the asymptotic part of an expression in the bracket; depending on the parameter $\lambda^{-1} \mathbb{C}$ as $|\lambda| \rightarrow \infty$. The substitution of expressions (22) into (23) easily yields 


$$
\frac{\partial \Psi}{\partial \tau_{k}^{(j)}}=\left[\left(\frac{\partial \Psi}{\partial \mathrm{x}}\right)_{0 j}^{-1} \psi^{(0)}(\lambda)^{k}\right]_{+} \frac{\partial \Psi}{\partial \lambda}+\sum_{s=1}^{n}\left[\left(\frac{\partial \Psi}{\partial \mathrm{x}}\right)_{s j}^{-1} \psi^{(0)}(\lambda)^{k}\right]_{+} \frac{\partial \Psi}{\partial x_{s}}(24)
$$

for all $k \in \mathbb{Z}_{+}, j=\overline{1, n}$. These relationships (24) comprise an infinite hierarchy of Lax-Sato compatible $[11,12]$ linear equations; where $(\ldots)_{+}$ denotes the asymptotic part of an expression in the bracket; depending on nonnegative powers of the complex parameter $\lambda \in \mathbb{C}$. As for the independent functional parameters $\tau_{k}^{(j)} \in C^{2}\left(\mathbb{R}^{\mathbb{Z}_{+}} \times \mathbb{T}^{n} ; \mathbb{R}\right)$ for all $k \in \mathbb{Z}_{+}, j=\overline{1, n}$, one can state their functional independence by taking into account their a priori linear dependence on the independent evolution parameters $t_{k} \in \mathbb{R}, k \in \mathbb{Z}_{+}$. On the other hand; taking into account the explicit form of the hierarchy of eqn. (24); following [13]; it is not hard to show that the corresponding vector fields

$$
\mathrm{A}_{k}^{(j)}:=\left[\left(\frac{\partial \Psi}{\partial \mathrm{x}}\right)_{0 j}^{-1} \psi^{(0)}(\lambda)^{k}\right]_{+} \frac{\partial}{\partial \lambda}+\sum_{s=1}^{n}\left[\left(\frac{\partial \Psi}{\partial \mathrm{x}}\right)_{s j}^{-1} \psi^{(0)}(\lambda)^{k}\right]_{+} \frac{\partial}{\partial x_{s}}
$$

on the manifold $\mathbb{C} \times \mathbb{T}^{n}$ satisfy for all $k, m \in \mathbb{Z}_{+}, j, l=\overline{1, n}$, the Lax-Sato compatibility conditions

$$
\frac{\partial \mathrm{A}_{m}^{(l)}}{\partial \tau_{k}^{(j)}}-\frac{\partial \mathrm{A}_{k}^{(j)}}{\partial \tau_{m}^{(l)}}=\left[\mathrm{A}_{k}^{(j)}, \mathrm{A}_{m}^{(l)}\right]
$$

which are equivalent to the independence of the all functional parameters $\tau_{k}^{(j)} \in C^{1}\left(\mathbb{R}^{\mathbb{Z}_{+}} \times \mathbb{T}^{n} ; \mathbb{R}\right), \quad k \in \mathbb{Z}_{+}, j=\overline{1, n}$. As a corollary of the analysis above; one can show that the infinite hierarchy of vector fields (18) is a linear combination of the basic vector fields (25) and also satisfies the Lax type compatibility condition (26). Inasmuch the coefficients of vector fields (25) are suitably smooth functions on the manifold $\mathbb{R}^{\mathbb{Z}_{+}} \times \mathbb{T}^{n}$, the compatibility conditions (26) yield the corresponding sets of differential-algebraic relationships on their coefficients; which have the common infinite set of invariants; thereby comprising an infinite hierarchy of completely integrable so called heavenly nonlinear dynamical systems on the corresponding multidimensional functional manifolds. That is; all of the above can be considered as an introduction to a recently devised [11-13,33] constructive algorithm for generating infinite hierarchies of completely integrable nonlinear dynamical systems of heavenly type on functional manifolds of arbitrary dimension. It is worthwhile to stress here that the above constructive algorithm for generating completely integrable nonlinear multidimensional dynamical systems still does not make it possible to directly show they are Hamiltonian and construct other related mathematical structures. This important problem is solved by employing other mathematical theories; for example; the analytical properties of the related loop diffeomorphisms groups generated by the hierarchy of vector fields (18).

Remark 2.2: The compatibility condition (26) allows an alternative differential-geometric description based on the Liealgebraic properties of the basic vector fields (25). Namely; consider the manifold $\mathbb{R}^{n \times \mathbb{Z}_{+}}$, as the base manifold of the vector bundle $E\left(\mathbb{R}^{n \times \mathbb{Z}_{+}}, G\right), \quad E=\cup_{\tau \in \mathbb{R}^{n \times \mathbb{Z}_{+}}}\left\{\left(G^{*} \otimes \tau\right) / \rho\right\}, \quad G^{*}:=\left\{\varphi^{*}: \varphi^{*} \beta^{(1)}:=\alpha^{(1)} \circ \varphi\right.$, $\beta^{(1)} \in \tilde{\Lambda}^{(1)}\left(\mathbb{C} \times \mathbb{T}^{n} ; \mathbb{C} \ngtr, \varphi \in G\right\}$ for an equivalence relation $\rho$ and the (holomorphic in $\lambda \in \mathbb{S}_{+}^{1} \cup \mathbb{S}_{-}^{1} \subset \mathbb{C} \not$ structure group $G=$ Diff $_{\text {hol }}\left(\mathbb{C} \mathbb{T}^{n}\right)$; naturally acting on the vector space $E$. The structure group can be endowed with a connection $\Upsilon$ by means of a mapping $d_{h}: \Gamma(E) \rightarrow \Gamma\left(T^{*}\left(\mathbb{R}^{n \times \mathbb{Z}_{+}}\right) \otimes E\right) \cong \Gamma\left(\operatorname{Hom}\left(T\left(\mathbb{R}^{n \times \mathbb{Z}_{+}}\right) ; E\right)\right)$, where

$$
\begin{gathered}
d_{h} \varphi_{\tau}^{*}:=\sum_{j \in \mathbb{Z}_{+}} d \tau_{j}^{(k)} \otimes \frac{\partial}{\partial \tau_{j}^{(k)}} \circ \varphi_{\tau}^{*}+\varphi_{\tau}^{*} \circ<\alpha^{(1)}, \frac{\partial}{\partial \mathrm{x}}>, \\
\alpha^{(1)}:=\sum_{j \in \mathbb{Z}_{+}} a_{j}^{(k)} d \tau_{j}^{(k)} \in \Lambda\left(\mathbb{R}^{n \times \mathbb{Z}_{+}}\right) \otimes \Gamma(E), \quad \text { which is defined for }
\end{gathered}
$$

any cotangent diffeomorphism $\varphi_{\tau}^{*} \in E, \quad \tau \in \mathbb{R}^{n \times \mathbb{Z}_{+}}$, generated by the set of parametric vector fields (25); and naturally acting on any mapping $\psi \in C^{2}\left(\mathbb{R}^{n \times \mathbb{Z}_{+}} \times\left(\mathbb{C} \times \mathbb{T}^{n} \not ; \mathbb{C}\right)\right.$ as $\varphi_{\tau}^{*} \circ \psi(\tau, \mathrm{x}):=\psi\left(\tau, \varphi_{\tau}(\mathrm{x})\right),(\tau, x) \in \mathbb{R}^{n \times \mathbb{Z}_{+}} \times \mathbb{T}^{n}$. It is easy now to see that the corresponding to (27) zero curvature condition $d_{h}^{2}=0$ is equivalent to the set of compatibility eqn. (26). Moreover; the parallel transport equation

$$
d_{h} \varphi_{\tau}^{*} \circ \psi=0
$$

coincides exactly with the infinite hierarchy of linear vector field eqn. (24); where $\psi \in C^{2}\left(\mathbb{R}^{n \times \mathbb{Z}_{+}} \times \mathbb{T}^{n} ; \mathbb{R}\right)$ is their invariant. Conversely; the Cartan integrable ideal of differential forms $h(\alpha) \in \Lambda\left(\mathbb{R}^{n \times \mathbb{Z}_{+}} \times \mathbb{T}^{n}\right) \otimes \Gamma\left(T^{*}\left(\mathbb{R}^{n \times \mathbb{Z}_{+}}\right)\right)$, which is equivalent to the zero curvature condition $d_{h}^{2}=0$, makes it possible to retrieve $[23,45]$ the corresponding connection $\Upsilon$ by constructing a mapping $d_{h}: \Gamma(E) \rightarrow \Gamma\left(T^{*}\left(\mathbb{R}^{n \times \mathbb{Z}_{+}}\right) \otimes E\right) \cong \Gamma\left(\operatorname{Hom}\left(T\left(\mathbb{R}^{n \times \mathbb{Z}_{+}}\right) ; E\right)\right)$ in the form $(27)$. These and other interesting related aspects of the integrable heavenly dynamical systems shall be investigated separately elsewhere.

\section{Example: the vector field representation for the Mikhalev- Pavlov heavenly type equation}

The Mikhalev-Pavlov equation was first constructed [32] and has the form

$$
u_{x t}+u_{y y}=u_{y} u_{x x}-u_{x} u_{x y},
$$

where $u \in C^{\infty}\left(\mathbb{R}^{2} \times \mathbb{T}^{1} ; \mathbb{R}\right)$ and $(t, y, x) \in \mathbb{R}^{2} \times \mathbb{T}^{1}$. Assume now [13] that the following two functions

$$
\psi^{(0)}=\lambda, \psi^{(1)} \sim \sum_{k=3}^{\infty} \lambda^{k} \tau_{k}-\lambda^{2} t+\lambda y+x+\sum_{j=1}^{\infty} \psi_{j}^{(1)}(t, y, \tau ; x) \lambda^{-j},
$$

where $\psi_{1}^{(1)}(t, y, \tau ; x) \stackrel{k=3}{=} u,(t, y, \tau ; x) \in \mathbb{R}^{2} \times \mathbb{R}^{\dot{i}=1} \times \mathbb{T}^{1}$, are invariants of the set of vector fields (19) for an infinite set of constant parameters $\tau_{k} \in \mathbb{R}, k=\overline{3, \infty}$, as the complex parameter $\lambda \rightarrow \infty$. By applying to the invariants (30) the criterion (23) in the form

$$
\left(\left(\partial \psi^{(1)} / \partial x\right)^{-1} \mathrm{~d} \psi^{(1)}\right)_{-}=0,
$$

one can easily obtain the following compatible linear vector field equations

$$
\begin{aligned}
& \frac{\partial \psi}{\partial t}+\left(\lambda^{2}+\lambda u_{x}-u_{y}\right) \frac{\partial \psi}{\partial x}=0 \\
& \frac{\partial \psi}{\partial y}+\left(\lambda+u_{x}\right) \frac{\partial \psi}{\partial x}=0, \\
& \ldots \\
& \frac{\partial \psi}{\partial \tau_{k}}+P_{k}(u ; \lambda) \frac{\partial \psi}{\partial x}=0,
\end{aligned}
$$

where $P_{k}(u ; \lambda), k=\overline{3, \infty}, \quad$ are independent differential-algebraic polynomials in the variable $u C^{\infty}\left(\mathbb{R}^{2} \times \mathbb{R}^{\infty} \times \mathbb{T}^{1}\right)$ and algebraic polynomials in the spectral parameter $\lambda \in \mathbb{C}$, calculated from the expressions (24). Moreover; as one can check; the compatibility condition (26) for the first two vector field eqn. (32) yields exactly the Mikhalev-Pavlov eqn. (29).

\section{Example: The Dunajski metric nonlinear equation}

The equations for the Dunajski metric [45] are

$$
\begin{aligned}
& u_{x_{1} t}+u_{y x_{2}}+u_{x_{1} x_{1}} u_{x_{2} x_{2}}-u_{x_{1} x_{2}}-v=0, \\
& v_{x_{1} t}+v_{x_{2} y}+u_{x_{1} x_{1}} v_{x_{2} x_{2}}-2 u_{x_{1} x_{2}} v_{x_{1} x_{2}}=0,
\end{aligned}
$$


where $(u, v) \in C^{\infty}\left(R^{2} \times T^{2} ; R^{2}\right),\left(y, t ; x_{1}, x_{2}\right) \in R^{2} \times T^{2}$. One can construct now; by definition; the following asymptotic expansions

$$
\begin{aligned}
& \psi^{(0)} \sim \lambda+\sum_{j=1}^{\infty} \psi_{j}^{(0)}(t, y ; x) \lambda^{-j}, \\
& \psi^{(1)} \sim \sum_{k=2}^{\infty}\left(\psi^{(0)}\right)^{k} \tau_{k}^{(1)}-\psi^{(0)} y+x_{1}+\sum_{j=1}^{\infty} \psi_{j}^{(1)}(t, y ; x)\left(\psi^{(0)}\right)^{-j}, \\
& \psi^{(2)} \sim \sum_{k=2}^{\infty}\left(\psi^{(0)}\right)^{k} \tau_{k}^{(2)}+\psi^{(0)} t+x_{2}+\sum_{j=1}^{\infty} \psi_{j}^{(1)}(t, y ; x)\left(\psi^{(0)}\right)^{-j},
\end{aligned}
$$

where $\partial u / \partial x_{1}:=\psi_{1}^{(2)}, \partial u / \partial x_{2}:=\psi_{1}^{(1)}, v:=\psi_{1}^{(0)}$ and $\tau_{k}^{(s)} \in R, s=\overline{1,2}, k=\overline{2, \infty}$, are constant parameters. Then the condition (23)

$$
\left(\left|\frac{\partial\left(\psi^{(0)}, \psi^{(1)}, \psi^{(2)}\right)}{\partial\left(\lambda, x_{1}, x_{2}\right)}\right|^{-1} \mathrm{~d} \psi^{(0)} \wedge \mathrm{d} \psi^{(1)} \wedge \mathrm{d} \psi^{(2)}\right)_{-}=0
$$

yield a compatible hierarchy of the following Lax-Sato type linear vector field equations:

$$
\begin{gathered}
A^{\left(t_{0}\right)} \psi:=\frac{\partial \psi}{\partial t}+\mathrm{A}^{\left(t_{0}\right)} \psi=0, \mathrm{~A}^{\left(t_{0}\right)}:=u_{x_{2} x_{2}} \frac{\partial}{\partial x_{1}}-\left(\lambda+u_{\left.x_{1} x_{2}\right)} \frac{\partial}{\partial x_{2}}+v_{x_{2}} \frac{\partial}{\partial \lambda}=0,\right. \\
A^{\left(t_{1}\right)} \psi:=\frac{\partial \psi}{\partial y}+\mathrm{A}^{\left(t_{1}\right)} \psi=0, \mathrm{~A}^{\left(t_{1}\right)}:=\left(\lambda-u_{x_{1} x_{2}}\right) \frac{\partial}{\partial x_{1}}+u_{x_{1} x_{1}} \frac{\partial}{\partial x_{2}}-v_{x_{1}} \frac{\partial}{\partial \lambda}=0, \\
A^{\left(t_{k}^{(s)}\right)} \psi:=\frac{\partial \psi}{\partial \tau_{k}^{s}}+<P_{k}^{s}(u ; \lambda), \frac{\partial \psi}{\partial \mathrm{x}}>=0,
\end{gathered}
$$

where $P_{k}^{s}(u, v ; \lambda) \in \mathbb{E}^{3}, s=\overline{1,2}, k \in \mathbb{N} \backslash\{1\}, \quad$ are some independent vector-valued differential-algebraic polynomials [33] in the variables $(u, v) \in C^{\infty}\left(\mathbb{R}^{2} \times \mathbb{R}^{\mathbb{Z}_{+}} \times \mathbb{T}^{2} ; \mathbb{R}^{2}\right)$ and algebraic polynomials in the spectral parameter $\lambda \in \mathbb{C}$, calculated from the expressions (24). In particular; the compatibility condition (26) for the first two equations of (35) is equivalent to the Dunajski metric nonlinear eqn. (33).

The description of the Lax-Sato equations presented above; especially their alternative differential-geometric interpretation (27) and (28); makes it possible to realize that the structure group Diff ${ }_{\text {hol }}\left(\mathbb{C} \mathbb{T}^{n}\right)$ should play an important role in unveiling the hidden Liealgebraic nature of the integrable heavenly dynamical systems. This is actually the case; and a detailed analysis is presented in the sequel.

\section{Heavenly Equations: The Lie-Algebraic Integrability Scheme}

Let $\left.\tilde{G}_{ \pm}:=\widetilde{\operatorname{Diff}}+\mathbb{T}^{n}\right), n \in \mathbb{Z}_{+}$, be subgroups of the loop diffeomorphisms group $\widetilde{\operatorname{Diff}}\left(\mathbb{T}^{n}\right):=\left\{\mathbb{C} \supset \mathbb{S}^{1} \rightarrow \operatorname{Diff}\left(\mathbb{T}^{n}\right)\right\}$, holomorphically extended in the interior $\mathbb{S}_{+}^{1} \subset \mathbb{C}$ and in the exterior $\mathbb{S}_{-}^{1} \subset \mathbb{C}$ regions of the unit circle $\mathbb{S}^{1} \subset \mathbb{C}^{1}$, such that for any $g(\lambda) \in \tilde{G}_{ \pm}, \lambda \in \mathbb{S}_{-}^{1}, g(\infty)=1 \in \operatorname{Diff}\left(\mathbb{T}^{n}\right)$. The corresponding Lie subalgebras $\tilde{\mathcal{G}}_{+}:=\widetilde{\operatorname{diff}}\left(\mathbb{T}^{n}\right)$ of the loop subgroups $\tilde{G}_{ \pm}$are vector fields on $\mathbb{T}^{n}$ holomorphic; respectively; on $\mathbb{S}_{ \pm}^{1} \subset \mathbb{C}^{1}$, where for any $\tilde{a}(\lambda) \in \tilde{\mathcal{G}}_{-}$the value $\tilde{a}(\infty)=0$. The split loop Lie algebra $\tilde{\mathcal{G}} \quad \tilde{\mathcal{G}}_{+}+\tilde{\mathcal{G}_{-}}$can be naturally identified with a dense subspace of the dual space $\tilde{\mathcal{G}}^{*}$ through the pairing

$$
(\tilde{l}, \tilde{a}):=\frac{1}{2 \pi i} \oint_{\mathbb{S}^{1}}(l(x, \lambda), a(x, \lambda))_{H^{q}} \frac{d \lambda}{\lambda^{p}},
$$

for some fixed $p, q \in \mathbb{Z}_{+}$. We took above; by definition [29,42]; a loop vector field $\tilde{a} \in \Gamma\left(\tilde{T}\left(\mathbb{T}^{n}\right)\right)$ and a loop differential 1-form $\tilde{l} \in \tilde{\Lambda}^{1}\left(\mathbb{T}^{n}\right)$ given as

$$
\tilde{a}=\sum_{j=1}^{n} a^{(j)}(x, \lambda) \frac{\partial}{\partial x_{j}}:=\left\langle a(x ; \lambda), \frac{\partial}{\partial x}\right\rangle,
$$

$$
\tilde{l}=\sum_{j=1}^{n} l_{j}(x, \lambda) d x_{j}:=\langle l(x ; \lambda), d x\rangle
$$

introduced for brevity the gradient operator $\frac{\partial}{\partial x}:=\left(\frac{\partial}{\partial x_{1}}, \frac{\partial}{\partial x_{2}}, \ldots, \frac{\partial}{\partial x_{n}}\right)^{\top}$ in the Euclidean space $\mathbb{E}^{n}$ and chose the Sobolev type metric $(\cdot, \cdot)_{H^{q}}$ on the space $C^{\infty}\left(\mathbb{T}^{n} ; \mathbb{R}^{n}\right) \subset H^{q}\left(\mathbb{T}^{n} ; \mathbb{R}^{n}\right)$ for some $q \in \mathbb{Z}_{+}$as

$$
(l(x ; \lambda), a(x ; \lambda))_{H^{q}}:=\sum_{j=1|\alpha|=0}^{n} \sum_{\mathbb{T}^{n}}^{q} d x\left(\frac{\partial^{|\alpha|} l_{j}(x ; \lambda)}{\partial x^{\alpha}} \frac{\partial^{|\alpha|} a^{(j)}(x ; \lambda)}{\partial x^{\alpha}}\right),
$$

where $\partial x^{\alpha}:=\partial x_{1}^{\alpha_{1}} \partial x_{2}^{\alpha_{2}} \ldots \partial x_{2}^{\alpha_{n}},|\alpha|=\sum_{j=1}^{n} \alpha_{j}$ for $\alpha \in \mathbb{Z}_{+}^{n}$, generalized metric used before [46]. The Lie commutator of vector fields $\tilde{a}, \tilde{b} \in \tilde{\mathcal{G}}$ is calculated the standard way and equals

$$
\begin{aligned}
& \tilde{a}, \tilde{b}]=\tilde{a} \tilde{b}-\tilde{b} \tilde{a}=\left\langle\left\langle a(x ; \lambda), \frac{\partial}{\partial x}\right\rangle b(x ; \lambda), \frac{\partial}{\partial x}\right\rangle- \\
& -\left\langle\left\langle b(x ; \lambda), \frac{\partial}{\partial x}\right\rangle a(x ; \lambda), \frac{\partial}{\partial x}\right\rangle .
\end{aligned}
$$

The Lie algebra $\tilde{\mathcal{G}}$ naturally splits into the direct sum of two Lie subalgebras

$$
\tilde{\mathcal{G}}=\tilde{\mathcal{G}}_{+} \oplus \tilde{\mathcal{G}_{-}},
$$

for which one can identify the dual spaces

$$
\tilde{\mathcal{G}}_{+}^{*} \simeq \lambda^{p-1} \tilde{\mathcal{G}}_{-}, \quad \tilde{\mathcal{G}}_{-}^{*} \simeq \lambda^{p-1} \tilde{\mathcal{G}}_{+},
$$

where for any $l(\lambda) \in \tilde{\mathcal{G}}_{-}^{*}$ one has the constraint $\tilde{l}(0)=0$. Having defined now the projections

$$
P_{ \pm} \tilde{\mathcal{G}}:=\tilde{\mathcal{G}} \pm \tilde{\mathcal{G}},
$$

one can construct a classical $\mathcal{R}$-structure $[25,26,47]$ on the Lie algebra $\tilde{\mathcal{G}}$ as the endomorphism $\mathcal{R}: \tilde{\mathcal{G}} \rightarrow \tilde{\mathcal{G}}$, where

$$
\mathcal{R}:=\left(P_{+}-P_{-}\right) / 2 \text {, }
$$

which allows to determine on the vector space $\tilde{\mathcal{G}}$ the new Lie algebra structure

$$
\tilde{a}, \tilde{b}]_{\mathcal{R}}:=[\mathcal{R} \tilde{a}, \tilde{b}]+[\tilde{a}, \mathcal{R} \tilde{b}]
$$

for any $\tilde{a}, \tilde{b} \in \tilde{\mathcal{G}}$, satisfying the standard Jacobi identity.

Let $\left.\mathrm{D} \boxplus \tilde{\mathcal{G}}^{*}\right)$ denote the space of smooth functions on $\tilde{\mathcal{G}}^{*}$. Then for any $\left.f, g \in \mathcal{D} \boxplus \tilde{\mathcal{G}}^{*}\right)$ one can write the canonical $[23,25,26,45]$ LiePoisson bracket

$$
\{f, g\}:=(\tilde{l},[\nabla f(\tilde{l}), \nabla g(\tilde{l})]),
$$

where $\tilde{l} \in \tilde{\mathcal{G}}^{*}$ is a seed element and $\nabla f, \nabla g \in \tilde{\mathcal{G}}$ are the standard functional gradients at $\tilde{l} \in \tilde{\mathcal{G}}^{*}$ with respect to the metric (37). The related to (45) space $\left.I \boxplus \tilde{\mathcal{G}}^{*}\right)$ of Casimir invariants is defined as the set $\left.I\left(\tilde{\mathcal{G}}^{*}\right) \subset \mathrm{D} \boxplus \tilde{\mathcal{G}}^{*}\right)$ of smooth independent functions $\left.h_{j} \in \mathrm{D} \boxplus \tilde{\mathcal{G}}^{*}\right), j=\overline{1, n}$, for which

$$
a d_{\nabla h_{j}(\tilde{l})}^{*} \tilde{l}=0,
$$

where for any seed element

$$
\tilde{l}=<l, d x>
$$

the gradients

$$
\nabla h_{j}(\tilde{l}):=\left\langle\nabla h_{j}(l), \frac{\partial}{\partial x}\right\rangle
$$


Citation: Blackmore D, Hentosh EO, Prykarpatski AK (2017) The Novel Lie-Algebraic Approach to Studying Integrable Heavenly Type MultiDimensional Dynamical Systems. J Generalized Lie Theory Appl 11: 286. doi: 10.4172/1736-4337.1000286

Page 6 of 19

and the coadjoint action (46) can be equivalently rewritten; for instance; in the case $q=0$, as

$$
\left\langle\frac{\partial}{\partial x}, \nabla h_{j}(l)\right\rangle l+\left\langle l,\left(\frac{\partial}{\partial x} \nabla h_{j}(l)\right)\right\rangle=0
$$

for any $j=\overline{1, n}$. If one takes two smooth functions $h^{(y)}, h^{(t)} \in I\left(\tilde{\mathcal{G}}^{*}\right) \subset \mathrm{D}\left(\tilde{\mathcal{G}}^{*}\right)$, their second Poisson bracket

$$
\left\{h^{(y)}, h^{(t)}\right\}_{\mathcal{R}}:=\left(\tilde{l},\left[\nabla h^{(y)}, \nabla h^{(t)}\right]_{\mathcal{R}}\right)
$$

on the space $\tilde{\mathcal{G}}^{*}$ vanishes; that is

$$
\left\{h^{(y)}, h^{(t)}\right\}_{\mathcal{R}}=0
$$

at any seed element $\tilde{l} \in \tilde{\mathcal{G}}^{*}$. Since the functions $h^{(y)}, h^{(t)} \in I\left(\tilde{\mathcal{G}}^{*}\right)$, the following coadjoint action relationships hold:

$$
a d_{\nabla h^{(y)}(\tilde{l})}^{*} \tilde{l}=0, \quad a d_{\nabla h^{(t)}(\tilde{l})}^{*} \tilde{l}=0,
$$

which can be equivalently rewritten (as above in the case $q=0$ ) as

$$
\begin{gathered}
\left\langle\frac{\partial}{\partial x}, \nabla h^{(y)}(l)\right\rangle l+\left\langle l,\left(\frac{\partial}{\partial x} \nabla h^{(y)}(l)\right)\right\rangle= \\
=\left\langle\nabla h^{(y)}(l), \frac{\partial}{\partial x}\right\rangle l+\left\langle\left(\frac{\partial}{\partial x}, \nabla h^{(y)}(l)\right)\right\rangle l+\left\langle l,\left(\frac{\partial}{\partial x} \nabla h^{(y)}(l)\right)\right\rangle:= \\
=\left(\nabla h^{(y)}(\tilde{l})+B_{\nabla h^{(y)}}\right) l
\end{gathered}
$$

and similarly

$$
\left\langle\frac{\partial}{\partial x}, \nabla h^{(t)}(l)\right\rangle l+\left\langle l,\left(\frac{\partial}{\partial x}, \nabla h^{(t)}(l)\right)\right\rangle:=\left(\nabla h^{(t)}(\tilde{l})+B_{\nabla h^{(t)}}\right) l,
$$

where the expressions

$$
\nabla h^{(y)}(\tilde{l}):=\left\langle\nabla h^{(y)}(l), \frac{d}{d x}\right\rangle, \nabla h^{(t)}(\tilde{l}):=\left\langle\nabla h^{(t)}(l), \frac{\partial}{\partial x}\right\rangle
$$

are true vector fields on $\mathbb{T}^{n}$, yet the expressions

$$
\begin{aligned}
& B_{\nabla h^{(y)}}:=\left\langle\left(\frac{\partial}{\partial x}, \nabla h^{(y)}(l)\right)\right\rangle+\left(\frac{\partial}{\partial x} \nabla h^{(y)}(l)\right), \\
& B_{\nabla h^{(t)}}:=\left\langle\left(\frac{\partial}{\partial x}, \nabla h^{(t)}(l)\right)\right\rangle+\left(\frac{\partial}{\partial x} \nabla h^{(t)}(l)\right),
\end{aligned}
$$

are the usual matrix homomorphisms of the Euclidean space $\mathbb{E}^{n}$.

Consider now the following Hamiltonian flows on the space $\tilde{\mathcal{G}}^{*}$ :

$$
\begin{aligned}
& \partial \tilde{l} / \partial y:=\left\{h^{(y)}, \tilde{l}\right\}_{\mathcal{R}}=-a d_{\nabla h^{(y)}(\tilde{l})_{+}}^{*} \tilde{l}, \\
& \partial \tilde{l} / \partial t:=\left\{h^{(t)}, \tilde{l}\right\}_{\mathcal{R}}=-a d_{\nabla h^{(t)}(\tilde{l})_{+}}^{*} \tilde{l},
\end{aligned}
$$

where $h^{(y)}, h^{(t)} \in I\left(\tilde{\mathcal{G}}^{*}\right)$ and $y, t \in \mathbb{R}$ are the corresponding evolution parameters. Since $h^{(y)}, h^{(t)} \in I\left(\tilde{\mathcal{G}}^{*}\right)$ are Casimirs; the flows (57) commute. Thus; taking into account the representations (53); one can recast the flows (57) as

$$
\partial l / \partial t=-\left(\nabla h^{(t)}(\tilde{l})_{+}+B_{\nabla h_{+}^{(t)}}\right) l, \quad \partial l / \partial y=-\left(\nabla h^{(y)}(\tilde{l})++B_{\nabla h_{+}^{(y)}}\right) l,
$$

where

$$
\nabla h^{(t)}(\tilde{l})_{+}:=\left\langle\nabla h^{(t)}(l)_{+}, \frac{\partial}{\partial x}\right\rangle, \quad \nabla h^{(y)}(\tilde{l})_{+}:=\left\langle\nabla h^{(y)}(l)_{+}, \frac{\partial}{\partial x}\right\rangle .
$$

Lemma 3.1: The compatibility of commuting flows (58) is equivalent to the Lax type vector fields relationship

$$
\frac{\partial}{\partial y} \nabla h^{(t)}(\tilde{l})_{+}-\frac{\partial}{\partial t} \nabla h^{(y)}(\tilde{l})_{+}+\left[\nabla h^{(y)}(\tilde{l})_{+}, \nabla h^{(t)}(\tilde{l})_{+}\right]=0,
$$

which holds for all $y, t \in \mathbb{R}$ and arbitrary $\lambda \in \mathbb{C}$.

Proof. The compatibility of commuting flows (58) implies that $\partial^{2} l / \partial t \partial y-\partial^{2} l / \partial y \partial t=0$ for all $y, t \in \mathbb{R}$ and arbitrary $\lambda \in \mathbb{C}$. Taking into account the expressions (57); one has for any vector field $\tilde{Z}=<Z, \frac{\partial}{\partial x}>\in \tilde{\mathcal{G}}$

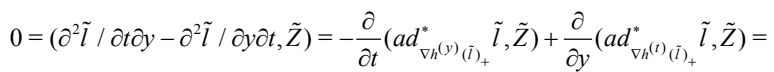

$$
\begin{aligned}
& =-\frac{\partial}{\partial t}\left(\tilde{l},\left[\nabla h^{(y)}(\tilde{l})_{+}, \tilde{Z}\right]\right)+\frac{\partial}{\partial y}\left(\tilde{l},\left[\nabla h^{(y)}(\tilde{l})_{+}, \tilde{Z}\right]\right)= \\
& =-\left(\frac{\partial}{\partial t} \tilde{l},\left[\nabla h^{(y)}(\tilde{l})_{+}, \tilde{Z}\right]\right)-\left(\tilde{l},\left[\frac{\partial}{\partial t} \nabla h^{(y)}(\tilde{l})_{+}, \tilde{Z}\right]\right)+ \\
& +\left(\frac{\partial}{\partial y} \tilde{l},\left[\nabla h^{(t)}(\tilde{l})_{+}, \tilde{Z}\right]\right)+\left(\tilde{l},\left[\frac{\partial}{\partial y} \nabla h^{(y)}(\tilde{l})_{+}, \tilde{Z}\right]\right)= \\
& =\left(a d_{\nabla h^{(t)}(\tilde{l})_{+}}^{*} \tilde{l},\left[\nabla h^{(y)}(\tilde{l})_{+}, \tilde{Z}\right]\right)-\left(\tilde{l},\left[\frac{\partial}{\partial t} \nabla h^{(y)}(\tilde{l})_{+}, \tilde{Z}\right]\right)- \\
& -\left(a d_{\nabla h}^{*(y)(\tilde{I})_{+}} \underset{l}{l},\left[\nabla h^{(t)}(\tilde{l})_{+}, \tilde{Z}\right]\right)+\left(\tilde{l},\left[\frac{\partial}{\partial y} \nabla h^{(y)}(\tilde{l})_{+}, \tilde{Z}\right]\right)= \\
& =\left(\tilde{l},\left[\nabla h^{(t)}(\tilde{l})_{+},\left[\nabla h^{(y)}(\tilde{l})_{+}, \tilde{Z}\right]\right]\right)-\left(\tilde{l},\left[\frac{\partial}{\partial t} \nabla h^{(y)}(\tilde{l})_{+}, \tilde{Z}\right]\right)- \\
& -\left(\tilde{l},\left[\nabla h^{(y)}(\tilde{l})_{+},\left[\nabla h^{(t)}(\tilde{l})_{+}, \tilde{Z}\right]\right]\right)+\left(\tilde{l},\left[\frac{\partial}{\partial y} \nabla h^{(t)}(\tilde{l})_{+}, \tilde{Z}\right]\right)= \\
& =\left(\tilde{l},\left[\nabla h^{(t)}(\tilde{l})_{+},\left[\nabla h^{(y)}(\tilde{l})_{+}, \tilde{Z}\right]\right]-\left[\nabla h^{(y)}(\tilde{l})_{+},\left[\nabla h^{(t)}(\tilde{l})_{+}, \tilde{Z}\right]\right]\right)+ \\
& +\left(\tilde{l},\left[\frac{\partial}{\partial y} \nabla h^{(t)}(\tilde{l})_{+}-\frac{\partial}{\partial t} \nabla h^{(y)}(\tilde{l})_{+}, \tilde{Z}\right]\right)= \\
& =\left(\tilde{l},\left[\nabla h^{(y)}(\tilde{l})_{+}, \nabla h^{(t)}(\tilde{l})_{+}\right]+\frac{\partial}{\partial y} \nabla h^{(t)}(\tilde{l})_{+}-\frac{\partial}{\partial t} \nabla h^{(y)}(\tilde{l})_{+}, \tilde{Z}\right)=
\end{aligned}
$$$$
=\left(\tilde{l},\left[\frac{\partial}{\partial y} \nabla h^{(t)}(\tilde{l})_{+}-\frac{\partial}{\partial t} \nabla h^{(y)}(\tilde{l})_{+}+\left[\nabla h^{(y)}(\tilde{l})_{+}, \nabla h^{(t)}(\tilde{l})_{+}\right], Z\right]\right)=\left(a d_{\nabla \tilde{H}}^{*} \tilde{l}, \tilde{Z}\right),
$$

where

$$
Q(\tilde{l}):=\frac{\partial}{\partial y} \nabla h^{(t)}(\tilde{l})_{+}-\frac{\partial}{\partial t} \nabla h^{(y)}(\tilde{l})_{+}+\left[\nabla h^{(y)}(\tilde{l})_{+}, \nabla h^{(t)}(\tilde{l})_{+}\right] .
$$

From (61) we obtain that $a d_{Q(\tilde{l})}^{*} \tilde{l}=0$ for all $y, t \in \mathbb{R}$ and arbitrary $\lambda \in \mathbb{C}$; that is the vector field (62) is the gradient of an analytical Casimir type vector field $Q(\tilde{l}) \in \tilde{\mathcal{G}}$. Now based on the analyticity of the vector field expression (62); one easily shows [13] that $Q(\tilde{l})=0$, thus finishing the proof.

For the exact representatives of the functions $h^{(y)}, h^{(t)} \in I\left(\tilde{\mathcal{G}}^{*}\right)$; it is necessary to solve the determining eqn. (49); taking into account that if the chosen element $\tilde{l} \in \tilde{\mathcal{G}}^{*}$ is singular as $|\lambda| \rightarrow \infty$, the related expansion

$$
\nabla h^{(p)}(l) \simeq \lambda^{p} \sum_{j \in \mathbb{Z}_{+}} \nabla h_{j}(l) \lambda^{-j},
$$

where the degree $p \in \mathbb{Z}_{+}$can be taken as arbitrary. Upon substituting (63) into (49) one can find recurrently all the coefficients $\nabla h(l)_{j}, j \mathbb{Z}_{+}$, 
Citation: Blackmore D, Hentosh EO, Prykarpatski AK (2017) The Novel Lie-Algebraic Approach to Studying Integrable Heavenly Type MultiDimensional Dynamical Systems. J Generalized Lie Theory Appl 11: 286. doi: 10.4172/1736-4337.1000286

Page 7 of 19

and then construct functional gradients of the Casimir functions $h^{(y)}, h^{(t)} \in I\left(\tilde{\mathcal{G}}^{*}\right)$ projected on $\tilde{\mathcal{G}}_{+}$as

$$
\nabla h^{(t)}(l)_{+}=\left(\lambda^{p_{t}} \nabla h(l)\right)_{+}, \quad \nabla h^{(y)}(l)_{+}=\left(\lambda^{p_{y}} \nabla h(l)\right)_{+}
$$

for some positive integers $p_{y} p_{t} \in \mathbb{Z}_{+}$.

Remark 3.2: As mentioned above; the expansion (63) is effective if a chosen seed element $\tilde{l} \in \tilde{\mathcal{G}}^{*}$ is singular as $|\lambda| \rightarrow \infty$. In the case when it is singular as $|\lambda| 0$, the expression (63) should be replaced by the expansion

$$
\nabla h^{(p)}(l) \sim \lambda^{-p} \sum_{j \in \mathbb{Z}_{+}} \nabla h_{j}^{(p)}(l) \lambda^{j}
$$

for an arbitrary $p \in \mathbb{Z}_{+}^{+}$, and the projected Casimir function gradients then are given by the expressions

$$
\nabla h^{(y)}(l)_{-}=\lambda\left(\lambda^{-p_{y}-1} \nabla h(l)\right)_{-}, \quad \nabla h^{(t)}(l)_{-}=\lambda\left(\lambda^{-p_{t}-1} \nabla h(l)\right)_{-}
$$

for some positive integers $p_{y} p_{t} \mathbb{Z}_{+}$. Then the corresponding flows are; respectively; written as

$$
\partial \tilde{l} / \partial t=a d_{\nabla h^{(t)}(\tilde{l})_{-}}^{*} \tilde{l}, \partial \tilde{l} / \partial y=a d_{\nabla h^{(y)}(\tilde{l})_{-}}^{*} \tilde{l} .
$$

The above results; owing to 3.1 ; can be formulated as the following main proposition.

Proposition 3.3: Let a seed vector field $\tilde{l} \in \tilde{\mathcal{G}}^{*}$ and $h^{(y)}, h^{(t)} \in I\left(\tilde{\mathcal{G}}^{*}\right)$ be Casimir functions subject to the metric $(\cdot, \cdot)$ on the loop Lie algebra $\tilde{\mathcal{G}}$ and the natural coadjoint action on the loop co-algebra $\tilde{\mathcal{G}}^{*}$. Then the following dynamical systems

$$
\partial \tilde{l} / \partial y=-a d_{\nabla h}^{*}{ }_{(y)}^{(\tilde{l})_{+}}, \tilde{l}, \partial \tilde{l} / \partial t=-a d_{\nabla h^{(t)}(\tilde{l})_{+}}^{*} \tilde{l}
$$

are commuting Hamiltonian flows for all $y, t \in \mathbb{R}$. Moreover; the compatibility condition of these flows is equivalent to the so called vector fields representation

$$
\left(\partial / \partial t+\nabla h^{(t)}(\tilde{l})_{+}\right) \psi=0, \quad\left(\partial / \partial y+\nabla h^{(y)}(\tilde{l})_{+}\right) \psi=0,
$$

where $\psi \in C^{\infty}\left(\mathbb{R}^{2} \times \mathbb{T}^{n} ; \mathbb{C}\right)$ and the vector fields $\nabla h^{(y)}(\tilde{l})_{+}, \nabla h^{(t)}(\tilde{l})_{+} \in \tilde{\mathcal{G}}_{+}$, given by the expressions (59) and (64); satisfy the Lax relationship (60).

The proposition above makes it possible to describe in a very effective way the Bäcklund transformations between two solution sets to the dispersionless heavenly type equations resulting from the Lax compatibility condition (60). Namely; let a diffeomorphism $\xi \in \widetilde{\operatorname{Diff}}\left(\mathbb{T}^{n}\right)$, depending parametrically on $\lambda, \mu \in \mathbb{C}$ and evolution variables $(y, t) \in \mathbb{R}^{2}$ be such that a seed loop differential form $\tilde{l}(x ; \lambda, \mu) \in \tilde{\mathcal{G}}^{*} \simeq \tilde{\Lambda}^{1}\left(\mathbb{T}^{n}\right)$ satisfies the invariance condition

$$
\tilde{l}(\xi(x ; \lambda, \mu) ; \lambda)=k \tilde{l}(x ; \mu)
$$

for some non-zero constant $k \mathbb{C} \backslash\{0\}$, any $x \mathbb{T}^{n}$ and arbitrarily chosen $\lambda \in \mathbb{C}$. As the seed element $\tilde{l}(\xi(x ; \lambda, \mu) ; \lambda) \in \tilde{\Lambda}^{1}\left(\mathbb{T}^{n}\right)$, by the construction; simultaneously satisfies the system of compatible equations following from (68); the loop diffeomorphism $\xi \in \widetilde{\operatorname{Diff}}\left(\mathbb{T}^{n}\right)$, found analytically from the invariance condition (70); should satisfiy the relationships

$$
\frac{\partial}{\partial y} \xi=\nabla h^{(y)}(l)_{+}, \frac{\partial}{\partial t} \xi=\nabla h^{(t)}(l)_{+},
$$

giving rise exactly to the Bäcklund type relationships for coefficients of the seed loop differential form ${ }_{x t}+u_{y y}=u_{y} u_{x x}-u_{x} u_{x y}$,

\section{Integrable Heavenly Type Equations: Examples}

\section{The Mikhalev-Pavlov heavenly type equation}

This equation $[20,32]$ is

$$
u_{x t}+u_{y y}=u_{y} u_{x x}-u_{x} u_{x y},
$$

where $u \in C^{\infty}\left(\mathbb{R}^{2} \times \mathbb{T}^{1}\right)$ and $(t, y ; x) \in \mathbb{R}^{2} \times \mathbb{T}^{1}$. Set $\tilde{\mathcal{G}}^{*}:=\widetilde{\operatorname{diff}}^{*}\left(\mathbb{T}^{1}\right)$ and take the corresponding seed element $\tilde{l} \in \tilde{\mathcal{G}}^{*}$ as

$$
\tilde{l}=\left(\lambda-2 u_{x}\right) d x \text {. }
$$

It generates a Casimir invariant $h \in I\left(\tilde{\mathcal{G}}^{*}\right)$ for which the expansion (63) as $|\lambda| \rightarrow \infty$ is given by the asymptotic series

$$
\nabla h(l) \sim 1+u_{x} / \lambda-u_{y} / \lambda^{2}+O\left(1 / \lambda^{3}\right)
$$

and so on. If further one defines

$$
\begin{aligned}
& \nabla h^{(t)}(l)_{+}:=\left(\lambda^{2} \nabla h\right)_{+}=\lambda^{2}+\lambda u_{x}-u_{y}, \\
& \nabla h^{(y)}(l)_{+}:=\left(\lambda^{1} \nabla h\right)_{+}=\lambda+u_{x},
\end{aligned}
$$

it is easy to verify that

$$
\begin{aligned}
& \nabla h^{(t)}(\tilde{l})_{+}:=<\nabla h^{(t)}(l)_{+}, \frac{\partial}{\partial x}>=\left(\lambda^{2}+\lambda u_{x}-u_{y}\right) \frac{\partial}{\partial x}, \\
& \nabla h^{(y)}(\tilde{l})_{+}:=<\nabla h^{(y)}(l)_{+}, \frac{\partial}{\partial x}>=\left(\lambda+u_{x}\right) \frac{\partial}{\partial x} .
\end{aligned}
$$

As a result of (76) and the commuting flows (68) on $\tilde{\mathcal{G}}^{*}$ we retrieve (the equivalent to the Mikhalev-Pavlov [20] ) eqn. (72) vector field compatibility relationships

$$
\frac{\partial \psi}{\partial t}+\left(\lambda^{2}+\lambda u_{x}-u_{y}\right) \frac{\partial \psi}{\partial x}=0=\frac{\partial \psi}{\partial y}+\left(\lambda+u_{x}\right) \frac{\partial \psi}{\partial x},
$$

satisfied for $\psi \in C^{2}\left(\mathbb{R}^{2} \times \mathbb{T}^{1} ; \mathbb{C}\right)$, any $(y, t ; x) \in \mathbb{R}^{2} \times \mathbb{T}^{1}$ and all $\lambda \in \mathbb{C}$.

We now study the Bäcklund transformation for two special solutions $u, \tilde{u} \in C^{2}\left(\mathbb{T}^{1} \times \mathbb{R}^{2} ; \mathbb{R}\right)$ to the Mikhalev-Pavlov eqn. (72). Let us consider a loop diffeomorphism $\xi \in \widetilde{\operatorname{Diff}}\left(\mathbb{T}^{1}\right)$ that is the mapping $\mathbb{T}^{1} \ni x \rightarrow \tilde{x}=\xi(x ; y, t, \lambda) \in \mathbb{T}^{1}$, which parametrically depends on $\lambda \in \mathbb{C}^{1}$ and the evolution variables $(y, t) \in \mathbb{R}^{2}$, satisfying the invariance condition (70) for the seed loop differential form (73):

$$
\left[\lambda-2 \tilde{u}_{\tilde{x}}(\tilde{x} ; t, y)\right] d \tilde{x}=\left[\lambda-2 u_{x}(x ; t, y)\right] d x,
$$

where for simplicity; we define $\mu=\in \mathbb{C}$ and the constant parameter $k=1$. From (78) one easily finds that

$$
\lambda \xi_{x}(x ; t, y)=2[\tilde{u}(\tilde{x} ; t, y)-u(x ; t, y)]_{x}+\lambda,
$$

or; equivalently;

$$
\xi(x ; \lambda)=x+2(\tilde{u}-u) / \lambda+\alpha(y, t ; \lambda),
$$

where $k \in \mathbb{C} \backslash\{0\}$ and $\alpha \in C^{2}\left(\mathbb{R}^{2} ; \mathbb{R}\right)$ is some mapping. As the loop diffeomorphism (79) should simultaneously satisfy the vector field eqn. (71); giving rise (at $\alpha(y, t, \lambda)=0)$ to the following Bäcklund type differential relationships

$$
2\left(\tilde{u}_{y}-u_{y}\right) / \lambda=\lambda+\tilde{u}_{\tilde{x}}, \quad 2\left(\tilde{u}_{t}-u_{t}\right) / \lambda=\lambda^{2}+\lambda \tilde{u}_{\tilde{x}}-\tilde{u}_{y},
$$

which hold for any $\lambda \in \mathbb{C}$ and two special solutions $u, \tilde{u} \in C^{2}\left(\mathbb{T}^{1} \times \mathbb{R}^{2} ; \mathbb{R}\right)$ to the Mikhalev-Pavlov eqn. (72).

\section{Example: The Witham heavenly type equation}

Consider the following [48-52] heavenly type equation:

$$
u_{t y}=u_{x} u_{x y}-u_{y} u_{x x},
$$

where $u \in C^{2}\left(\mathbb{R}^{2} \times \mathbb{T}^{1} ; \mathbb{R}\right)$ and $(t, y ; x) \in \mathbb{R}^{2} \times \mathbb{T}^{1}$. To prove the Lax-Sato type integrability of (80); let us consider a seed element $\tilde{l} \in \tilde{\mathcal{G}}^{*}$, defined as

$$
\tilde{l}=\left(u_{y}^{-2} \lambda^{-1}+2 u_{x}+\lambda\right) d x,
$$


Citation: Blackmore D, Hentosh EO, Prykarpatski AK (2017) The Novel Lie-Algebraic Approach to Studying Integrable Heavenly Type MultiDimensional Dynamical Systems. J Generalized Lie Theory Appl 11: 286. doi: 10.4172/1736-4337.1000286

Page 8 of 19

where $\lambda \in \mathbb{C}$ is a complex parameter. The following asymptotic expressions are gradients of the Casimir functionals $h^{(t)}, h^{(y)} \in I\left(\tilde{\mathcal{G}}^{*}\right)$, related with the holomorphic loop Lie algebra $\tilde{\mathcal{G}}=\widetilde{\operatorname{diff}}\left(\mathbb{T}^{1}\right)$ :

$$
\nabla h^{(t)}(l) \sim \lambda\left(u_{x} \lambda^{-1}-1\right)+O(1 / \lambda),
$$

as $\lambda \rightarrow \infty$ and

$$
\nabla h^{(y)}(l) \sim u_{y} \lambda^{-1}+O\left(\lambda^{2}\right)
$$

as $\lambda \rightarrow 0$ Based on the expressions (82) and (83); one can construct [29] the following commuting to each other Hamiltonian flows

$$
\frac{\partial}{\partial y} \tilde{l}=-a d_{\nabla h}^{*(y)}(\tilde{l})_{-}, \frac{\partial}{\partial t} \tilde{l}=-a d_{\nabla h^{(t)}(\tilde{l})_{+}}^{*} \tilde{l}
$$

with respect to the evolution parameters $y, t \in \mathbb{R}$, which give rise; in part; to the following equations:

$$
\begin{aligned}
& u_{y t}=u_{x} u_{x y}-u_{y} u_{x x}, \\
& u_{t}=-u_{y}^{-2} / 2+3 u_{x}^{2} / 2, \\
& u_{y y}=-u_{y}^{3}\left[\left(u_{x} u_{y}\right)_{x}+u_{x} u_{x y}\right],
\end{aligned}
$$

where the projected gradients $\nabla h_{-}^{(y)}, \nabla h_{+}^{(t)} \in \tilde{\mathcal{G}}$ are equal to the loop vector fields

$$
\nabla h^{(t)}(\tilde{l})_{+}=\left(u_{x}-\lambda\right) \frac{\partial}{\partial x}, \nabla h^{(y)}(\tilde{l})_{-}=\frac{u_{y}}{\lambda} \frac{\partial}{\partial x},
$$

satisfying for evolution parameters $y, t \in \mathbb{R}^{2}$ the Lax-Sato vector field compatibility condition:

$$
\frac{\partial}{\partial y} \nabla h^{(y)}(\tilde{l})_{+}-\frac{\partial}{\partial t} \nabla h^{(y)}(\tilde{l})_{-}+\left[\nabla h^{(t)}(\tilde{l})_{+}, \nabla h^{(y)}(\tilde{l})_{-}\right]=0 .
$$

As a simple consequence of the condition one finds exactly the first equation of the (85); coinciding with the heavenly type eqn. (80). Thereby; we have stated that this equation is a completely integrable heavenly type dynamical system with respect to both evolution parameters.

Remark 4.1: It is worth to observe that the third equation of (85) entails the interesting relationship

$$
\frac{\partial}{\partial y}\left(1 / u_{y}\right)=\frac{\partial}{\partial x}\left(u_{x} u_{y}^{2}\right)
$$

whose compatibility makes it possible to introduce a new function $v C^{2}\left(\mathbb{S}^{1} ; \mathbb{R}\right)$, satisfying the next differential expressions:

$$
v_{x}=1 / u_{y}, \quad v_{y}=u_{x} u_{y}^{2},
$$

which hold for all $(x, y) \in \mathbb{S}^{1} \times \mathbb{R}$. Based on (89) the seed element (81) is rewritten as

$$
\tilde{l}=\left(v_{x}^{2} \lambda^{-1}+2 u_{x}+\lambda\right) d x
$$

and the vector fields (86) are rewritten as

$$
\nabla h^{(t)}(\tilde{l})_{+}=\left(u_{x}-\lambda\right) \frac{\partial}{\partial x}, \nabla h^{(y)}(\tilde{l})_{-}=\frac{1}{v_{x} \lambda} \frac{\partial}{\partial x},
$$

whose compatibility condition (87) gives rise to the following system of heavenly type nonliner integrable flows:

$$
\begin{aligned}
& v_{y}=u_{x} v_{x}^{-2}, v_{x t}=u_{x} v_{x y}+u_{x x} v_{x}, \\
& u_{y}=1 / v_{x}, u_{t}=-v_{x}^{2} / 2+3 u_{x}^{2} / 2,
\end{aligned}
$$

compatible for arbitrary evolution parameters $y, t \in \mathbb{R}$.

\section{The Hirota heavenly equation}

The Hirota equation describes $[41,53]$ three-dimensional Veronese webs and reads as

$$
\alpha u_{x} u_{y t}+\beta u_{y} u_{x t}+\gamma u_{t} u_{x y}=0
$$

for any evolution parameters $t, y \in \mathbb{R}$ and the spatial variable $x \mathbb{T}^{1}$, where $\alpha, \beta$ and $\gamma \in \mathbb{R}$ are arbitrary constants; satisfying the numerical constraint

$$
\alpha+\beta+=0 \text {. }
$$

To demonstrate the Lax-type integrability of the Hirota eqn. (93) we choose a seed vector field $\tilde{l} \in \tilde{\mathcal{G}}^{*}:=\overline{\operatorname{diff}}^{*}\left(\mathbb{R}^{1}\right)$ in the following rational form

$$
\tilde{l}=\left(\frac{u_{x}^{2}}{u_{t}^{2}(\lambda+\alpha)}-\frac{u_{x}^{2}\left(u_{y}^{2}+u_{t}^{2}\right)}{2 \alpha u_{t}^{2} u_{y}^{2}}+\frac{u_{x}^{2}}{u_{y}^{2}(\lambda-\alpha)}\right) d x .
$$

The corresponding gradients for the Casimir invariants $\gamma^{(j)} \in I\left(\tilde{\mathcal{G}}^{0}\right)$, $j=\overline{1,2}$, are given by the following asymptotic expansions:

$$
\nabla \gamma^{(1)}(l) \sim \sum_{j \in \mathbb{Z}_{+}} \nabla \gamma_{j}^{(1)}(l) \mu^{j},
$$

as $\lambda+\alpha:=\mu \rightarrow 0$, and

$$
\nabla \gamma^{(2)}(l) \sim \sum_{j \in \mathbb{Z}_{+}} \nabla \gamma_{j}^{(2)}(l) \mu^{j},
$$

as $\lambda-\alpha=\rightarrow 0$. For the first case (96) one easily obtains that

$$
\nabla \gamma^{(1)}(l) \sim-2 \gamma \frac{u_{t}}{u_{x}}+O\left(\mu^{2}\right)
$$

and for the second one (97) one obtains

$$
\nabla \gamma^{(2)}(l) \sim 2 \beta \frac{u_{y}}{u_{x}}+O\left(\mu^{2}\right),
$$

where we took into account that the following two Hamiltonian flows on $\tilde{\mathcal{G}}^{*}$

$$
d \tilde{l} / d y=a d_{\nabla h^{(t)}(\tilde{l})_{-}}^{*} \tilde{l}, \quad d \tilde{l} / d t=a d_{\nabla h^{(t)}(\tilde{l})_{-}}^{*} \tilde{l}
$$

with respect to the evolution parameters $y, t \in \mathbb{R}$ hold for the following conservation laws gradients:

$$
\begin{aligned}
& \nabla h^{(t)}(l)_{-}:=\left.\mu\left(\mu^{-2} \nabla \gamma^{(1)}(l)\right)_{-}\right|_{\mu=\lambda+\alpha}=\frac{-2 \gamma}{(\lambda+\alpha)} \frac{u_{t}}{u_{x}}, \\
& \nabla h^{(y)}(l)_{-}:=\left.\mu\left(\mu^{-2} \nabla \gamma^{(2)}(l)\right)_{-}\right|_{\mu=\lambda-\alpha}=\frac{2 \beta}{(\lambda-\alpha)} \frac{u_{y}}{u_{x}} .
\end{aligned}
$$

It is easy now to check that the compatibility (108) for a set of the vector fields (109) gives rise to the Hirota heavenly eqn. (93); whose equivalent Lax-Sato vector field representation reads as a system of the linear vector field equations

$$
\frac{\partial \psi}{\partial t}-\frac{2 \gamma u_{t}}{u_{x}(\lambda+\alpha)} \frac{\partial \psi}{\partial x}=0, \frac{\partial \psi}{\partial y}+\frac{2 \beta u_{y}}{u_{x}(\lambda-\alpha)} \frac{\partial \psi}{\partial x}=0,
$$

satisfied for $\psi \in C^{2}\left(\mathbb{R}^{2} \times \mathbb{T}^{1} ; \mathbb{C}\right)$ for all $u_{y t}+u_{t} u_{x y}-u_{x t} u_{y}=0$ and $\lambda \in C \backslash\{ \pm \alpha\}$.

\section{The first reduced Shabat type heavenly equation}

The entitled above equation [40] reads as

$$
u_{y t}+u_{t} u_{x y}-u_{x t} u_{y}=0
$$

for a function $u \in C^{\infty}\left(\mathbb{R}^{2} \times \mathbb{R}^{1} ; \mathbb{R}\right)$, where $(y, t ; x) \in \mathbb{R}^{2} \times \mathbb{R}^{1}$. To show the LaxSato integrability of the eqn. (103); take a seed element $\tilde{l} \in \tilde{\mathcal{G}}^{*}:=\widetilde{\text { diff }}^{*}\left(\mathbb{R}^{1}\right)$ in the following form:

$$
\tilde{l}=\left(\frac{u_{t}^{-2}}{\lambda+1}+\frac{u_{t}^{2}-u_{y}^{2}}{u_{y}^{2} u_{t}^{2}}+\frac{u_{y}^{-2}}{\lambda}\right) d x,
$$

where $\lambda \in \mathbb{C} \backslash\{0,-1\}$. This element generates two independent hierarchies of Casimir functionals $\gamma^{(1)}, \gamma^{(2)} \in I\left(\tilde{\mathcal{G}}^{*}\right)$, whose gradient expansions are given by the following asymptotic expansions: 
Citation: Blackmore D, Hentosh EO, Prykarpatski AK (2017) The Novel Lie-Algebraic Approach to Studying Integrable Heavenly Type MultiDimensional Dynamical Systems. J Generalized Lie Theory Appl 11: 286. doi: 10.4172/1736-4337.1000286

$$
\nabla \gamma^{(1)}(l) \sim u_{t}+O\left(\mu^{2}\right)
$$

as $\lambda+1:=\mu \rightarrow 0$; and

$$
\nabla \gamma^{(2)}(l) \sim u_{y}+O\left(\mu^{2}\right),
$$

as $\lambda:=\mu \rightarrow 0$. Having put now; by definition;

$$
\nabla h^{(t)}(l)_{-}:=\left.\mu\left(\mu^{-2} \nabla \gamma^{(1)}(l)\right)_{-}\right|_{\mu=\lambda+1}, \nabla h^{(y)}(l)_{-}:=\left.\mu\left(\mu^{-2} \nabla \gamma^{(2)}(l)\right)_{-}\right|_{\mu=\lambda},
$$

one easily ensues from the compatibility condition

$$
\frac{\partial}{\partial t} \nabla h^{(y)}(\tilde{l})_{-}-\frac{\partial}{\partial y} \nabla h^{(t)}(\tilde{l})_{-}=\left[\nabla h^{(y)}(\tilde{l})_{-}, \nabla h^{(t)}(\tilde{l})_{-}\right],
$$

for a set of the vector fields

$$
\nabla h^{(t)}(\tilde{l})_{-}:=\nabla h_{-}^{(t)}(l)_{+} \frac{\partial}{\partial x}, \nabla h^{(y)}(\tilde{l})_{+}:=\nabla h_{-}^{(y)}(l) \frac{\partial}{\partial x}
$$

a compatible Lax-Sato representation as the following system of vector field equations:

$$
\frac{\partial \psi}{\partial t}+\frac{u_{t}}{\lambda+1} \frac{\partial \psi}{\partial x}=0, \quad \frac{\partial \psi}{\partial y}+\frac{u_{y}}{\lambda} \frac{\partial \psi}{\partial x}=0,
$$

satisfied for $\psi \in C^{\infty}\left(\mathbb{R}^{2} \times \mathbb{R}^{1} ; \mathbb{C}\right)$, any $(t, y ; x) \in \mathbb{R}^{2} \times \mathbb{R}^{1}$ and all $\lambda \in \mathbb{C} \backslash$ $\{0,-1\}$.

\section{The second reduced Shabat type heavenly equation}

The entitled above equation [40] reads as

$$
u_{y y}-u_{x t} u_{y}+u_{t} u_{x y}=0
$$

for a function $u \in C\left(\mathbb{R}^{2} \times \mathbb{R}^{1} ; \mathbb{R}\right)$, where $(y, t ; x) \in \mathbb{R}^{2} \mathbb{T}^{1}$. In this case for demonstrating the Lax-Sato integrability of the eqn. (111) we will take a seed element $\tilde{l} \in \tilde{\mathcal{G}}^{*}:=\widetilde{\operatorname{diff}}^{*}\left(\mathbb{T}^{1}\right)$ as

$$
\tilde{l}=\left(\lambda u_{y}^{-2}+2\left(u_{t}+u_{y}^{2}\right) u_{y}^{-3}+\lambda^{-1} u_{t}\left(3 u_{t}+4 u_{y}\right) u_{y}^{-4}\right) d x,
$$

giving rise to two independent Casimir functionals $\gamma^{(1)}, \gamma^{(2)} \in I\left(\tilde{\mathcal{G}}^{*}\right)$, whose gradient expansions are given by the following asymptotic expansions

$$
\begin{aligned}
& \nabla \gamma^{(1)}(l) \sim-\lambda u_{y}+u_{t}+O\left(1 / \lambda^{2}\right), \\
& \nabla \gamma^{(2)}(l) \sim \lambda u_{y}-\left(u_{t}+u_{y}\right)+O\left(1 / \lambda^{2}\right)
\end{aligned}
$$

as $\lambda \rightarrow \infty$. Having put now; by definition;

$$
\begin{aligned}
& \nabla h_{+}^{(t)}(l):=\left.\left(\lambda \nabla \gamma^{(1)}(l)\right)\right|_{+}=\lambda u_{t}-\lambda^{2} u_{y}, \\
& \nabla h_{+}^{(y)}(l):=-\left(\lambda \nabla \gamma^{(1)}(l)+\left.\lambda \nabla \gamma^{(2)}(l)\right|_{+}=\lambda u_{y},\right.
\end{aligned}
$$

one obtains from (108) and (109) for the heavenly eqn. (103) the following compatible Lax-Sato vector field representation

$$
\frac{\partial \psi}{\partial t}+\left(\lambda u_{t}-\lambda^{2} u_{y}\right) \frac{\partial \psi}{\partial x}=0, \quad \frac{\partial \psi}{\partial y}+\lambda u_{y} \frac{\partial \psi}{\partial x}=0,
$$

satisfied for $\psi \in C\left(\mathbb{R}^{2} \times \mathbb{R}^{1} ; \mathbb{C}\right)$, any $(t, y ; x) \in \mathbb{R}^{2} \mathbb{R}^{1}$ and all $\lambda \in \mathbb{C}$.

\section{Pleban'ski heavenly equation}

This equation [16] is

$$
u_{t x_{1}}-u_{y x_{2}}+u_{x_{1} x_{1}} u_{x_{2} x_{2}}-u_{x_{1} x_{2}}^{2}=0
$$

for a function $u \in C\left(\mathbb{R}^{2} ; \mathbb{T}^{2}\right)$, where $\left(y, t ; x_{1}, x_{2}\right) \in \mathbb{R}^{2} \times \mathbb{T}^{2}$. We set $\tilde{\mathcal{G}}^{*}:=\widetilde{\operatorname{diff}}\left(\mathbb{T}^{2}\right)$ and take the corresponding seed element $\tilde{l} \in \tilde{\mathcal{G}}^{*}$ as

$$
\tilde{l}=\left(\lambda-u_{x_{1} x_{2}}+u_{x_{1} x_{1}}\right) d x_{1}+\left(\lambda-u_{x_{2} x_{2}}+u_{x_{1} x_{2}}\right) d x_{2} .
$$

This generates two independent Casimir functionals $h^{(1)}, h^{(2)} \in I\left(\tilde{\mathcal{G}}^{*}\right)$, whose gradient expansions (63) as $|\lambda| \rightarrow \infty$ are given by the expressions

$$
\begin{gathered}
\nabla h^{(1)}(l) \sim(0,1)^{\top}+\left(u_{x_{2} x_{2}},-u_{x_{1} x_{2}}\right)^{\top} \lambda^{-1}+O\left(\lambda^{-2}\right), \\
\nabla h^{(2)}(l) \sim(1,0)^{\top}+\left(u_{x_{1} x_{2}},-u_{x_{1} x_{2}}\right)^{\top} \lambda^{-1}+O\left(\lambda^{-2}\right),
\end{gathered}
$$

and so on. Now; by defining

$$
\begin{aligned}
& \nabla h^{(y)}(l)_{+}:=\left(\lambda \nabla h^{(1)}(l)\right)_{+}=\left(u_{x_{2} x_{2}}, \lambda-u_{x_{1} x_{2}}\right)^{\top}, \\
& \nabla h^{(t)}(l)_{+}:=\left(\lambda \nabla h^{(2)}(l)\right)_{+}=\left(\lambda+u_{x_{1} x_{2}},-u_{x_{1} x_{1}}\right)^{\top},
\end{aligned}
$$

one obtains for (116) the following [16] vector field representation

$$
\begin{aligned}
& \frac{\partial \psi}{\partial t}+u_{x_{1} x_{1}} \frac{\partial \psi}{\partial x_{1}}+\left(\lambda-u_{x_{1} x_{2}}\right) \frac{\partial \psi}{\partial x_{2}}=0, \\
& \frac{\partial \psi}{\partial z}+\left(\lambda+u_{x_{1} x_{2}}\right) \frac{\partial \psi}{\partial x_{1}}-u_{x_{1} x_{1}} \frac{\partial \psi}{\partial x_{2}}=0,
\end{aligned}
$$

satisfied for $\psi \in C^{2}\left(\mathbb{R}^{2} \times \mathbb{T}^{2} ; \mathbb{C}\right)$, any $\left(t, y ; x_{1}, x_{2}\right) \in \mathbb{R}^{2} \times \mathbb{T}^{2}$ and all $\lambda \in \mathbb{C}$.

\section{General heavenly equation}

This equation was first suggested and analyzed by Schief [21,53]; where it was shown to be equivalent to the first Pleba n'ski heavenly equation; and later studied by Doubrov and Ferapontov [54]; it has the form

$$
\alpha u_{y t} u_{x_{1} x_{2}}+\beta u_{t x_{2}} u_{y x_{1}}+\gamma u_{t x_{1}} u_{y x_{2}}=0,
$$

where $\alpha, \beta$ and $\gamma \in \mathbb{R}$ are arbitrary constants; satisfying the constraint

$$
\alpha+\beta+=0 \text {, }
$$

and $t, y \in \mathbb{R},\left(x_{1}, x_{2}\right) \in \mathbb{T}^{2}$. To demonstrate the Lax integrability of the eqn. (121) we choose now a seed vector field $\tilde{l} \in \tilde{\mathcal{G}}^{*}:=\widetilde{\operatorname{diff}}^{*}\left(\mathbb{T}^{2}\right)$ in the following rational form

$$
\begin{aligned}
& \tilde{l}=\left(\frac{\mu u_{x_{1} x_{2}}^{2}}{\gamma(\mu+\beta)}+\frac{u_{x_{1} x_{2}}^{2}}{\alpha}-\frac{\mu u_{x_{1} x_{2}}^{2}}{\beta(\mu-\gamma)}\right) d x_{1}+ \\
& +\left(\frac{\mu u_{x_{1} x_{2}} u_{x_{2} x_{2}}}{\gamma(\mu+\beta)}+\frac{u_{x_{1} x_{2}} u_{x_{2} x_{2}}}{\alpha}-\frac{\mu u_{x_{1} x_{2}} u_{x_{2} x_{2}}}{\beta(\mu-\gamma)}\right) d x_{2},
\end{aligned}
$$

where $a_{j}, b_{j} \in C^{\infty}\left(\mathbb{T}^{2} ; \mathbb{R}\right), \quad j=\overline{0,1}$, are smooth functions and $\mu \in \mathbb{C}$ is a complex parameter. The corresponding equations for independent Casimir invariants $h^{(j)} \in I\left(\tilde{\mathcal{G}}^{*}\right), j=\overline{1,2}$, are given with respect to the standard metric $(\cdot, \cdot)$ by the following asymptotic expansions:

$$
\nabla h^{(1)}(l) \sim \sum_{j \in \mathbb{Z}_{+}} \nabla h_{j}^{(1)}(l) \lambda^{j},
$$

as $\mu+\beta=\lambda \rightarrow 0$ and

$$
\nabla h^{(2)}(l) \sim \sum_{j \in \mathbb{Z}_{+}} \nabla h_{j}^{(2)}(l) \lambda^{j},
$$

as $\mu-\gamma=\rightarrow 0$. For the first case (124) one obtains that

$$
\nabla h^{(1)}(l) \sim\left(-\frac{\beta u_{t x_{2}}}{u_{x_{1} x_{2}}}+\frac{u_{t x_{2}}}{u_{x_{1} x_{2}}} \lambda, \frac{\beta u_{x_{1} x_{1}}}{u_{x_{1} x_{2}}}\right)^{\top}+O\left(\lambda^{2}\right)
$$

and for the second one (125) one finds that

$$
\nabla h^{(2)}(l) \sim\left(\frac{\gamma u_{y x_{2}}}{u_{x_{1} x_{2}}}+\frac{u_{y x_{2}}}{u_{x_{1} x_{2}}} \lambda,-\frac{\gamma u_{x_{1} x_{1}}}{u_{x_{1} x_{2}}}\right)^{\top}+O\left(\lambda^{2}\right) .
$$

Here we took into account that the following two Hamiltonian flows on $\tilde{\mathcal{G}}^{*}$ 
Citation: Blackmore D, Hentosh EO, Prykarpatski AK (2017) The Novel Lie-Algebraic Approach to Studying Integrable Heavenly Type MultiDimensional Dynamical Systems. J Generalized Lie Theory Appl 11: 286. doi: 10.4172/1736-4337.1000286

Page 10 of 19

$$
\partial \tilde{l} / \partial y=a d_{\nabla h}^{*}{ }^{(y)}(\tilde{l})_{-}, \quad \tilde{l} / \partial t=a d_{\nabla h^{(t)}(\tilde{l})_{-}}^{*} \tilde{l}
$$

with respect to the evolution narameters $y, t \in \mathbb{R}$ hold for the following conservation laws gradients:

$$
\begin{aligned}
& \nabla h^{(t)}(l)_{-}:=\lambda\left(\left.\lambda^{-2} \nabla h^{(1)}(l)_{-}\right|_{\lambda=\mu+\beta}=\left(\frac{\mu u_{t x_{2}}}{u_{x_{1} x_{2}}(\mu+\beta)}, \frac{\beta u_{t x_{1}}}{u_{x_{1} x_{2}}(\mu+\beta)}\right)^{\top},\right. \\
& \nabla h^{(y)}(l)_{-}:=\lambda\left(\left.\lambda^{-2} \nabla h^{(2)}(l)_{-}\right|_{\lambda=\mu-\gamma}=\left(\frac{\mu u_{y x_{2}}}{u_{x_{1} x_{2}}(\mu-\gamma)},-\frac{\gamma u_{y x_{1}}}{u_{x_{1} x_{2}}(\mu-\gamma)}\right)^{\top} .\right.
\end{aligned}
$$

Owing to the compatibility condition of two commuting flows (129); one can easily rewrite it as the Lax relationship

$$
\partial \nabla h^{(y)}(\tilde{l})_{-} / \partial t-\partial \nabla h^{(t)}(\tilde{l})_{-} / \partial y=\left[\nabla h^{(y)}(\tilde{l})_{-}, \nabla h^{(t)}(\tilde{l})_{-}\right],
$$

where

$$
\begin{aligned}
& \nabla h^{(t)}(\tilde{l})_{-}:=\left\langle\nabla h^{(t)}(l)_{-}, \frac{\partial}{\partial x}\right\rangle=\frac{\mu u_{t x_{2}}}{u_{x_{1} x_{2}}(\mu+\beta)} \frac{\partial}{\partial x_{1}}+\frac{\beta u_{t x_{1}}}{u_{x_{1} x_{2}}(\mu+\beta)} \frac{\partial}{\partial x_{2}}, \\
& \nabla h^{(y)}(\tilde{l})_{-}:=\left\langle\nabla h^{(y)}(l)_{-}, \frac{\partial}{\partial x}\right\rangle=\frac{\mu u_{y x_{2}}}{u_{x_{1} x_{2}}(\mu-\gamma)} \frac{\partial}{\partial x_{1}}-\frac{\gamma u_{y x_{1}}}{u_{x_{1} x_{2}}(\mu-\gamma)} \frac{\partial}{\partial x_{2}} .
\end{aligned}
$$

An easy calculation shows that the general heavenly eqn. (121) follows from the compatibility condition (130); whose equivalent vector field representation is given as

$$
\begin{aligned}
& \frac{\mu u_{t x_{2}}}{u_{x_{1} x_{2}}(\mu+\beta)} \frac{\partial \psi}{\partial x_{1}}+\frac{\beta u_{t x_{1}}}{u_{x_{1} x_{2}}(\mu+\beta)} \frac{\partial \psi}{\partial x_{2}}+\frac{\partial \psi}{\partial t}=0, \\
& \frac{\mu u_{y x_{2}}}{u_{x_{1} x_{2}}(\mu-\gamma)} \frac{\partial \psi}{\partial x_{1}}-\frac{\gamma u_{y x_{1}}}{u_{x_{1} x_{2}}(\mu-\gamma)} \frac{\partial \psi}{\partial x_{2}}+\frac{\partial \psi}{\partial y}=0
\end{aligned}
$$

for a function $\psi \in C^{2}\left(\mathbb{R}^{2} \times \mathbb{T}^{2} ; \mathbb{C}\right)$ for all $\left(y, t ; x_{1}, x_{2}\right) \in \mathbb{R}^{2} \times \mathbb{T}^{2}$

\section{The Alonso-Shabat heavenly type equation}

This equation [40] has the form

$u_{y x_{2}}-u_{t} u_{y x_{1}}+u_{y} u_{t x_{1}}=0$,

where $u \in C^{\infty}\left(\mathbb{R}^{2} \times \mathbb{T}^{2} ; \mathbb{R}\right),(y, t) \in \mathbb{R}^{2}$ and $\left(x_{1}, x_{2}\right) \in \mathbb{T}^{2}$. To prove its Lax integrability; we define a seed element $\tilde{l} \in \tilde{\mathcal{G}}^{*}:=\widetilde{\operatorname{diff}}{ }^{*}\left(\mathbb{T}^{2}\right)$ of the form

$$
\tilde{l}=z_{x_{1}}^{2}(\lambda+1) d x_{1}+z_{x_{1}} z_{x_{2}}(\lambda+1) d x_{2},
$$

for a fixed function $z C^{\infty}\left(\mathbb{T}^{2} ; \mathbb{R}\right)$. Then one easily obtains asymptotic expansionsas $|\lambda| \rightarrow \infty$ for coefficients of the two independent Casimir functionals $h^{(1)}, h^{(2)} \in I\left(\tilde{\mathcal{G}}^{*}\right)$ gradients:

$$
\begin{aligned}
& \nabla h^{(1)}(l) \sim\left(1 / z_{x_{1}}+k z_{x_{2}} / z_{x_{1}},-k\right)^{\top}+O\left(1 / \lambda^{2}\right), \\
& \nabla h^{(2)}(l) \sim\left(z_{x_{2}} / z_{x_{1}},-1\right)^{\top}+O\left(1 / \lambda^{2}\right),
\end{aligned}
$$

where $k \neq 1$ is a constant and $\alpha_{1}, \alpha_{2} C^{\infty}\left(\mathbb{T}^{1} ; \mathbb{R}\right)$ are different functions. Uing the Casimir functionals (135); one can construct the simplest two commuting flows

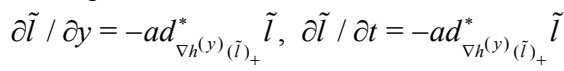

with respect to the evolution parameters $y, t \in \mathbb{R}$, where

$$
\begin{gathered}
\nabla h^{(y)}(l)_{+}:=\left(\lambda \nabla h_{1}(l)\right)_{+}=\left(\lambda / z_{x_{1}}+\lambda k z_{x_{2}} / z_{x_{1}},-\lambda k\right)^{\top}:=\left(\lambda u_{y},-\lambda k\right)^{\top}, \\
\nabla h^{(t)}(l)_{+}:=\left(\lambda \nabla h_{2}(l)\right)_{+}=\left(\lambda z_{x_{2}} / z_{x_{1}},-\lambda\right)^{\top}:=\left(\lambda u_{t},-\lambda\right)^{\top}
\end{gathered}
$$

for some function $u \in C^{\infty}\left(\mathbb{R}^{2} \times \mathbb{T}^{2} ; \mathbb{R}\right)$. From relationships (137); as a result of the commutativity of the flows (136); one derives the equivalent Lax type relationship (60) for the vector fields; namely

$$
\nabla h^{(y)}(\tilde{l})_{+}:=\lambda u_{y} \partial / \partial x_{1}-k \lambda \partial / \partial x_{2}, \nabla h^{(t)}(\tilde{l})_{+}:=\lambda u_{t} \partial / \partial x_{1}-\lambda \partial / \partial x_{2},
$$

which can be rewritten as the compatibility condition for the following vector field equations:

$$
\frac{\partial \psi}{\partial t}+\lambda u_{t} \frac{\partial \psi}{\partial x_{1}}-\lambda \frac{\partial \psi}{\partial x_{2}}=0, \frac{\partial \psi}{\partial y}+\lambda u_{y} \frac{\partial \psi}{\partial x_{1}}-k \lambda \frac{\partial \psi}{\partial x_{2}}=0
$$

satisfied for $\psi \in C^{2}\left(\mathbb{R}^{2} \times \mathbb{T}^{2} ; \mathbb{C}\right)$, any $\left(t, y ; x_{1}, x_{2}\right) \in \mathbb{R}^{2} \times \mathbb{T}^{2}$ and all $\lambda \in \mathbb{C}$. The resulting equation is then

$$
u_{y x_{2}}-u_{t} u_{y x_{1}}+u_{y} u_{t x_{1}}+k u_{t x_{2}}=0,
$$

which reduces at $k=0$ to the Alonso-Shabat heavenly eqn. (133).

Remark 4.2: It is interesting to observe that the seed elements $\tilde{l} \in \tilde{\mathcal{G}}^{*}$ of the examples presented above have the differential geometric structure:

$$
\tilde{l}=\eta d \rho,
$$

where $\eta$ and $\rho \in C^{\infty}\left(\mathbb{R}^{2} \times\left(\mathbb{C} \times \mathbb{T}^{2}\right) ; \mathbb{C}\right)$ are some smooth functions. For instance;

$$
\begin{aligned}
& \tilde{l}=d(\lambda x-2 u)-\text { Mikhalev--Pavlovtypeequation, } \\
& \tilde{l}=d\left(\lambda x_{1}+\lambda x_{2}-u_{x_{2}}+u_{x_{1}}\right)-\text { Pleban'skiheavenlyequation, } \\
& \left.\tilde{l}=u_{x_{1} x_{2}} \xi d u_{x_{2}}, \xi:=\left(\mu[\gamma(\mu+\beta)]^{-1}+\alpha^{-1}-\mu \beta(\mu-\gamma)\right]^{-1}\right)- \text { generalheavenlyequation, } \\
& \tilde{l}=(\lambda+1) z_{x_{1}} d z-\text { Alonso }-- \text { Shabatheavenlytypeequation. }
\end{aligned}
$$

\section{The Generalized Lie-Algebraic Structures and Related Heavenly Type Quasi-Hamiltonian Systems}

It is well known that the loop Lie algebra $\tilde{\mathcal{G}}:=\widetilde{\operatorname{diff}}\left(\mathbb{T}^{n}\right)$ can be centrally extended as $\hat{\mathcal{G}}:=\left(\widetilde{\operatorname{diff}}\left(\mathbb{T}^{n}\right) ; \mathbb{R}^{1}\right)$ only [55] for the case $n=1$, where for any two elements $(\tilde{a} ; \alpha)$ and $(\tilde{b} ; \beta) \in \hat{\mathcal{G}}$ the commutator

$$
(\tilde{a} ; \alpha),(\tilde{b} ; \beta)]=\left([\tilde{a}, \tilde{b}] ; \omega_{2}(\tilde{a}, \tilde{b})\right) \in \tilde{\mathcal{G}}
$$

nd the 2-cocycle $\omega_{2}: \tilde{\mathcal{G}} \times \tilde{\mathcal{G}} \rightarrow \mathbb{R}^{1}$ satisfies the condition

$$
\omega_{2}([\tilde{a}, \tilde{b}], \tilde{c})+\omega_{2}([\tilde{b}, \tilde{c}], \tilde{a})+\omega_{2}([\tilde{c}, \tilde{a}], \tilde{b})=0
$$

for any $\tilde{a}, \tilde{b}$ and $\tilde{c} \in \tilde{\mathcal{G}}$. For the case $n=1$; the Gelfand-Fuchs 2-cocycle [55] on the loop Lie algebra $\tilde{\mathcal{G}}$ equals the expression

$$
\omega_{2}(\tilde{a}, \tilde{b})=\frac{1}{2 \pi i} \oint_{\mathbb{S}^{1}}\left(\frac{\partial^{2} a(x ; \lambda)}{\partial x^{2}}, \frac{\partial b(x ; \lambda)}{\partial x}\right)_{H^{0}} \frac{d \lambda}{\lambda^{p}}
$$
for any vector fields $\tilde{a}=a(x ; \lambda) \frac{\partial}{\partial x}, \tilde{b}=b(x ; \lambda) \frac{\partial}{\partial x} \in \tilde{\mathcal{G}}$ on $\mathbb{T}^{1}$ and a fixed
integer $p \in \mathbb{Z}$.

TheThe integrable dynamical systems related to this central extension were described in detail [47]. Concerning a further generalization of the multi-dimensional case related to the loop group $\tilde{\mathcal{G}}$ for $n \mathbb{Z}_{+}$one can proceed in the following natural way: as the Lie algebra $\tilde{\mathcal{G}} \widetilde{\operatorname{diff}}\left(\mathbb{T}^{n}\right)$ consists of the elements formally depending additionally on the "spectral" variable $\lambda \in \mathbb{C}^{1}$, one can extend the basic Lie structure on $\mathcal{G} \operatorname{diff}\left(\mathbb{T}^{n}\right)$ to that on the adjacent holomorphic in $\lambda \in \mathbb{S}_{ \pm}^{1}$ Lie algebra $\overline{\mathcal{G}}:=\operatorname{diff}_{\text {hol }}\left(\mathbb{C} \times \mathbb{T}^{n}\right) \subset \operatorname{diff}\left(\mathbb{C} \times \mathbb{T}^{n}\right)$ of vector fields on $\mathbb{C T}^{n}$. This has elements representable as $\bar{a}(x ; \lambda):=<a(x ; \lambda), \frac{\partial}{\partial \mathrm{x}}>=\sum_{j=1}^{n} a_{j}(x ; \lambda) \frac{\partial}{\partial x_{j}}+a_{0}(x ; \lambda) \frac{\partial}{\partial \lambda} \in \overline{\mathcal{G}}$ for some holomorphic in $\lambda \in \mathbb{S}_{ \pm}^{1}$ vectors $a(x ; \lambda) \in \mathbb{E} \times \mathbb{E}^{n}$ for all $x \in \mathbb{T}^{n}$, where $\frac{\partial}{\partial \mathrm{x}}:=\left(\frac{\partial}{\partial \lambda}, \frac{\partial}{\partial x_{1}}, \frac{\partial}{\partial x_{2}}, \ldots, \frac{\partial}{\partial x_{n}}\right)^{\top}$ is the generalized Euclidean vector gradient with respect to the vector variable $\mathrm{x}:=(\lambda, x) \in \mathbb{C} \times \mathbb{T}^{n}$. 
It is now important to mention that the Lie algebra $\overline{\mathcal{G}} \subset \operatorname{diff}\left(\mathbb{C} \times \mathbb{T}^{n}\right)$ also splits into the direct sum of two subalgebras:

$$
\overline{\mathcal{G}}=\overline{\mathcal{G}}_{+} \oplus \overline{\mathcal{G}}_{-},
$$

allowing to introduce on it the classical $\mathcal{R}$-structure:

$$
\bar{a}, \bar{b}]_{\mathcal{R}}:=[\mathcal{R} \bar{a}, \bar{b}]+[\bar{a}, \mathcal{R} \bar{b}]
$$

for any $\bar{a}, \bar{b} \in \overline{\mathcal{G}}$, where

$$
\mathcal{R}:=\left(P_{+}-P_{-}\right) / 2 \text {, }
$$

and

$$
P_{ \pm} \overline{\mathcal{G}}:=\overline{\mathcal{G}_{ \pm}} \subset \overline{\mathcal{G}} .
$$

The space $\overline{\mathcal{G}}^{*} \simeq \Lambda^{1}\left(\mathbb{C} \times \mathbb{T}^{n} \not\right.$, adjoint to the Lie algebra $\overline{\mathcal{G}}$ of vector fields on $\mathbb{C} \times \mathbb{T}^{n}$, can be functionally identified with $\overline{\mathcal{G}}$ subject to the Sobolev type metric

$$
(\bar{l}, \bar{a})=\frac{1}{2 \pi i} \oint_{\mathbb{S}^{1}} \lambda^{-p} d \lambda(l, a)_{H^{q}},
$$

where $p \in \mathbb{Z}, q \in \mathbb{Z}_{+}$and for arbitrary $\bar{l}:=<l(x ; \lambda), d \mathrm{x}>=\sum_{j=0, n} l_{j}(x ; \lambda) d \mathrm{x}_{j} \in \overline{\mathcal{G}}^{*}$, $\bar{a}=\sum_{j=0, n} a_{j}(x ; \lambda), \frac{\partial}{\partial \mathrm{x}_{j}} \in \overline{\mathcal{G}}$ one defines

$$
(l, a)_{H^{q}}=\sum_{j=0|\alpha|=0}^{n} \sum_{\mathbb{T}^{n}}^{q} d x \frac{\partial^{|\alpha|} l_{j}}{\partial \mathrm{x}^{\alpha}} \frac{\partial^{|\alpha|} a_{j}}{\partial \mathrm{x}^{\alpha}} .
$$

In particular; for $q=0$ one has

$$
(\bar{l}, \bar{a})=\frac{1}{2 \pi i} \oint_{\mathbb{S}^{1}} \lambda^{-p} \int_{\mathbb{T}^{n}} d x \sum_{j=0}^{n} l_{j} a_{j},
$$

the case which will be mainly chosen later on. Then for arbitrary $f, g \in \mathrm{D}\left(\overline{\mathcal{G}}^{*}\right)$, one can determine two Lie-Poisson brackets

$$
\{f, g\}:=(\bar{l},[\nabla f(\bar{l}), \nabla g(\bar{l})])
$$

and

$$
\{f, g\}_{\mathcal{R}}:=\left(\bar{l},[\nabla f(\bar{l}), \nabla g(\bar{l})]_{\mathcal{R}}\right),
$$

where at any seed element ${ }^{-} \epsilon^{-}$the gradient element $\nabla f(\bar{l})$ and $\nabla g(\bar{l}) \in \overline{\mathcal{G}}$ are calculated with respect to the metric (149).

Now let us assume that a smooth function $h \in I\left(\overline{\mathcal{G}}^{*}\right)$ is a Casimir invariant; that is

$$
a d_{\nabla h(\bar{l})}^{*} \bar{l}=0
$$

for a chosen seed element $\bar{l} \in \overline{\mathcal{G}}^{*}$. As the adjoint mapping $a d_{\nabla f(\bar{l})}^{*} \bar{l}$ for any $\left.\in \mathrm{D}^{-}\right)$can be rewritten in the reduced form as

$$
a d_{\nabla f(\bar{l})}^{*}(\bar{l})=\left\langle\frac{\partial}{\partial \mathrm{x}}, \nabla f(l)\right\rangle \bar{l}+\sum_{j=1}^{n}\left\langle\left\langle l, \frac{\partial}{\partial \mathrm{x}} \nabla f(l)\right\rangle, d \mathrm{x}\right\rangle,
$$

where $\nabla f(\bar{l}):=<\nabla f(l), \frac{\partial}{\partial \mathrm{x}}>$. For the Casimir function $h \in \mathrm{D}\left(\overline{\mathcal{G}}^{*}\right)$, the condition (154) is then equivalent to the equation

$$
\left\langle\frac{\partial}{\partial \mathrm{x}}, \nabla f(l)\right\rangle l+\left\langle l,\left(\frac{\partial}{\partial \mathrm{x}} \nabla h(l)\right)\right\rangle=0,
$$

which should be be solved analytically. In the case when an element $\bar{l} \in \overline{\mathcal{G}}^{*}$ is singular as $|\lambda| \rightarrow \infty$, one can consider the general asymptotic expansion

$$
\nabla h:=\nabla h^{(p)} \sim \lambda^{p} \sum_{j \in \mathbb{Z}_{+}} \nabla h_{j}^{(p)} \lambda^{-j}
$$

for some suitably chosen integers $p \in \mathbb{Z}_{+}$, and upon substituting (157) into the eqn. (156); one can solve it recurrently.
Now let $h^{(y)}, h^{(t)} \in I\left(\overline{\mathcal{G}}^{*}\right)$ be such Casimir functions for which the Hamiltonian vector field generators

$$
\nabla h^{(y)}(l)_{+}:=\left(\nabla h^{\left(p_{y}\right)}(l)\right)_{+}, \quad \nabla h^{(t)}(l)_{+}:=\left(\nabla h^{\left(p_{t}\right)}(l)\right)_{+}
$$

are; respectively; defined for special integers $p_{y} p_{t} \in \mathbb{Z}_{+}$. These invariants generate; owing to the Lie-Poisson bracket (153); for the case $q=0$ the following commuting flows

$$
\begin{aligned}
& \partial l / \partial t=-\left\langle\frac{\partial}{\partial \mathrm{x}}, \nabla h^{(t)}(l)_{+}\right\rangle l-\left\langle l,\left(\frac{\partial}{\partial \mathrm{x}} \nabla h^{(t)}(l)_{+}\right)\right\rangle, \\
& \partial l / \partial y=-\left\langle\frac{\partial}{\partial \mathrm{x}}, \nabla h^{(y)}(l)_{+}\right\rangle l-\left\langle l,\left(\frac{\partial}{\partial \mathrm{x}} \nabla h^{(y)}(l)_{+}\right)\right\rangle>,
\end{aligned}
$$

where $y, t \in \mathbb{R}$ are the corresponding evolution parameters. Since the invariants $h^{(y)}, h^{(t)} \in I\left(\overline{\mathcal{G}}^{*}\right)$ commute with respect to the Lie-Poisson bracket (153); the flows (159) also commute; implying that the corresponding Hamiltonian vector field generators

$$
\nabla h^{(t)}(\bar{l})_{+}:=\left\langle\nabla h^{(t)}(l)_{+}, \frac{\partial}{\partial \mathrm{x}}\right\rangle, \quad \nabla h^{(y)}(\bar{l})_{+}:=\left\langle\nabla h^{(y)}(l)_{+}, \frac{\partial}{\partial \mathrm{x}}\right\rangle
$$

satisfy the Lax compatibility condition

$$
\frac{\partial}{\partial y} \nabla h^{(t)}(\bar{l})_{+}-\frac{\partial}{\partial t} \nabla h^{(y)}(\bar{l})_{+}=\left[\nabla h^{(t)}(\bar{l})_{+}, \nabla h^{(y)}(\bar{l})_{+}\right]
$$

for all $y, t \in \mathbb{R}$. On the other hand; the condition (161) is equivalent to the compatibility condition of two linear equations

$$
\left(\frac{\partial}{\partial t}+\nabla h^{(t)}(\bar{l})_{+}\right) \psi=0, \quad\left(\frac{\partial}{\partial y}+\nabla h^{(y)}(\bar{l})_{+}\right) \psi=0
$$

for a function $\psi \in C^{2}\left(\mathbb{R} \times \mathbb{T}^{n} ; \mathbb{C}\right)$ for all $y, t \in \mathbb{R}$ and any $\lambda \in \mathbb{C}$.

The above can be formulated as the following key result:

Proposition 5.1: Let a seed vector field be $\bar{l} \in \overline{\mathcal{G}}^{*}$ and $h^{(y)}, h^{(t)} \in I\left(\overline{\mathcal{G}}^{*}\right)$ be Casimir functions subject to the metric $(\cdot, \cdot)$ on the loop Lie algebra $\overline{\mathcal{G}}$ and the natural coadjoint action on the loop co-algebra $\overline{\mathcal{G}}^{*}$. Then the following dynamical systems

$$
\partial \bar{l} / \partial y=-a d_{\nabla h^{(y)}(\bar{l})_{+}}^{*} \bar{l}, \partial \bar{l} / \partial t=-a d_{\nabla h^{(t)}(\bar{l})_{+}}^{*} \bar{l}
$$

are commuting Hamiltonian flows for all $y, t \in \mathbb{R}$. Moreover; the compatibility condition of these flows is equivalent to the vector fields representation

$$
\left(\partial / \partial t+\nabla h^{(t)}(\bar{l})_{+}\right) \psi=0, \quad\left(\partial / \partial y+\nabla h^{(y)}(\bar{l})_{+}\right) \psi=0,
$$

where $\psi \in C^{2}\left(\mathbb{R}^{2} \times \mathbb{C} \times \mathbb{T}^{n} ; \mathbb{C}\right)$ and the vector fields $\nabla h^{(t)}(\bar{l})_{+}, \nabla h^{(t)}(\bar{l})_{+} \in \overline{\mathcal{G}}_{+}$are given by the expressions (160) and (158).

Remark 5.2: As mentioned above; the expansion (157) is effective if a chosen seed element $\bar{l} \in \overline{\mathcal{G}}^{*}$ is singular as $|\lambda| \rightarrow \infty$. In the case when it is singular as $|\lambda| \rightarrow 0$, the expression (157) should be replaced by the expansion

$$
\nabla h^{(p)}(l) \sim \lambda^{-p} \sum_{j \in \mathbb{Z}_{+}} \nabla h_{j}^{(p)}(l) \lambda^{j}
$$

for suitably chosen integers $p \mathbb{Z}_{+}$, and the reduced Casimir function gradients then are given by the Hamiltonian vector field generators

$$
\nabla h^{(y)}(l)_{-}:=\lambda\left(\lambda^{-p_{y}-1} \nabla h^{\left(p_{y}\right)}(l)\right)_{-}, \quad \nabla h^{(t)}(l)_{-}:=\lambda\left(\lambda^{-p_{t}-1} \nabla h^{\left(p_{t}\right)}(l)\right)_{-}
$$

for suitably chosen positive integers $p_{y}, p_{t} \in Z_{+}$and the corresponding Hamiltonian flows are; respectively; written as

$$
\partial \bar{l} / \partial t=-a d_{\nabla h^{(t)}(\bar{l})_{-}}^{*} \bar{l}, \partial \bar{l} / \partial y=-a d_{\nabla h^{(y)}(\bar{l})_{-}}^{*} \bar{l} .
$$

As in Section 3 the Proposition 5.1 above makes it possible to 
Citation: Blackmore D, Hentosh EO, Prykarpatski AK (2017) The Novel Lie-Algebraic Approach to Studying Integrable Heavenly Type MultiDimensional Dynamical Systems. J Generalized Lie Theory Appl 11: 286. doi: 10.4172/1736-4337.1000286

describe the Bäcklund transformations between two special solution sets for the dispersionless heavenly equations resulting from the Lax compatibility condition (163). Let a diffeomorphism $\xi \in$ Diff $_{h o l}\left(\mathbb{C} \times \mathbb{T}^{n}\right)$ be such that a seed loop differential form $\bar{l}(\lambda, x) \in \overline{\mathcal{G}}^{*} \simeq \Lambda^{1}\left(\mathbb{C} \times \mathbb{T}^{n}\right)$ satisfies the invariance condition

$$
\bar{l}(\xi(\mathrm{x} ; \mu))=k \bar{l}(\overline{\mathrm{x}})
$$

for some non-zero constant $k \mathbb{C} \backslash\{0\}$, any $x=(\lambda, x)$ and $\overline{\mathrm{x}}=(\mu, x) \in \mathbb{C} \times \mathbb{T}^{n}$ and arbitrarily an chosen parameter $\mu \in \mathbb{C}$. As the seed element $\bar{l}(\xi(\mathrm{x} ; \mu)) \in \Lambda_{h o l}^{1}\left(\mathbb{C} \times \mathbb{T}^{n}\right)$ satisfies simultaneously the system of compatible equations (163); the loop diffeomorphism $\xi \in \operatorname{Diff}_{\text {hol }}\left(\mathbb{C} \times \mathbb{T}^{n}\right)$, found analytically from the invariance condition (168); satisfies the compatible system of vector field equations

$$
\frac{\partial}{\partial t} \xi=\nabla h_{+}^{(t)}(l), \frac{\partial}{\partial y} \xi=\nabla h_{+}^{(y)}(l)
$$

giving rise to the Bäcklund type relationships for the coefficients of the seed loop differential form $\bar{l} \in \overline{\mathcal{G}}^{*} \simeq \Lambda_{\text {hol }}^{1}\left(\mathbb{C} \times \mathbb{T}^{n}\right)$.

It worth mentionoing that; following Ovsienko's scheme [18,19]; one can consider a wider class of integrable heavenly equations; realized as compatible Hamiltonian flows on the semidirect product of the holomorphic loop Lie algebra $\tilde{\mathcal{G}}$ of vector fields on the torus $\mathbb{T}^{n}$ and its regular co-adjoint space $\tilde{\mathcal{G}}^{*}$, supplemented with naturally related cocycles. We plan to analyze this aspect of the construction; devised in the present study; in a separate work under preparation (60). Heavenly type equations: the modified Lie-algebraic integrability scheme

The rest of this Section is devoted to describing the Lie algebraic structure and integrability properties of a generalized hierarchy of the Lax-Sato type compatible systems of vector field equations and related nonlinear dynamical systems; based on the AKS-algebraic and related $\mathcal{R}$-structure schemes [22-26]; recently devised in B. Szablikowski and A. Sergyeyev [37,38]; V. Ovsienko [18,19] and also in refs. [28,29]. The devised there technique we apply to the holomorphic metrized loop Lie algebra $\tilde{\mathcal{G}}:=\widetilde{\operatorname{diff}}\left(\mathbb{T}^{n}\right) \ltimes \widetilde{\operatorname{diff}}\left(\mathbb{T}^{n}\right)^{*}$, the semidirect sum of the loop Lie algebra $\widetilde{\operatorname{diff}}\left(\mathbb{T}^{n}\right):=\widetilde{\operatorname{Vect}}\left(\mathbb{T}^{n}\right)$ of vector fields on the torus $\mathbb{T}^{n}, n \in \mathbb{Z}$, and its dual space $\widetilde{\operatorname{diff}}\left(\mathbb{T}^{n}\right)^{*}$, studying the orbits of the coadjoint actions on the dual space $\tilde{\mathcal{G}}^{*} \simeq \tilde{\mathcal{G}}$, related with the classical Lie-Poisson type structures on them. This construction; being centrally extended; makes it possible to construct commuting to each other Hamiltonian flows on the suitably extended adjoint space $\breve{\mathcal{G}}^{*}$, generated by a suitably chosen seed element $\tilde{a} \ltimes \tilde{l} \in \overline{\mathcal{G}}^{*}$ and the related Casimir invariants. We successively demonstrate that their compatibility condition; being equivalent to that for a system of specially constructed three linear LaxSato type vector field equations; gives rise to an infinite hierarchy of new heavenly type Hamiltonian flows; interesting for applications.

Let $\widetilde{\operatorname{Diff}_{ \pm}}\left(\mathbb{T}^{n}\right), n \in \mathbb{Z}_{+}$, be subgroups of the loop diffeomorphisms group $\widetilde{\operatorname{Diff}}\left(\mathbb{T}^{n}\right):=\left\{\mathbb{C} \supset \mathbb{S}^{1} \rightarrow \operatorname{Diff}\left(\mathbb{T}^{n}\right)\right\}, \quad$ holomorphically extended; respectively; on the interior $\mathbb{S}_{+}^{1} \subset \mathbb{C}$ and on the exterior $\mathbb{S}_{-}^{1} \subset \mathbb{C}$ regions of the unit circle $\mathbb{S}^{1} \subset \mathbb{C}^{1}$, such that for any $g(\lambda) \in \widetilde{\operatorname{Diff}}\left(\mathbb{T}^{n}\right)$, $\lambda \in \mathbb{S}_{-}^{1}, \quad g(\infty)=1 \in \operatorname{Diff}\left(\mathbb{T}^{n}\right)$. The corresponding Lie subalgebras $\widetilde{\operatorname{diff}_{+}}\left(\mathbb{T}^{n}\right) \simeq \widetilde{\operatorname{Vect}}_{ \pm}\left(\mathbb{T}^{n}\right)$ of the loop subgroups $\widetilde{\text { Diff }_{ \pm}}\left(\mathbb{T}^{n}\right)$ are vector fields on $\mathbb{T}^{n}$ holomorphic; respectively; on $\mathbb{S}_{+}^{1} \subset \mathbb{C}^{1}$, where for any $\tilde{a}(\lambda) \in \widetilde{\text { diff }}\left(\mathbb{T}^{n}\right)$ the value $\tilde{a}(\infty)=0$. The split loop Lie algebra $\tilde{\mathcal{G}} \tilde{\mathcal{G}}_{+}+\tilde{\mathcal{G}}_{-}$can be naturally identified with a dense subspace of the dual space $\widetilde{\operatorname{diff}}\left(\mathbb{T}^{n}\right)^{*}$ through the pairing

$$
(\tilde{l}, \tilde{a})_{1}:=\frac{1}{2 \pi i} \oint_{\mathbb{S}^{1}} \frac{d \lambda}{\lambda}(l(x ; \lambda), a(x ; \lambda))_{H^{0}}
$$

for any elements $\tilde{l} \in \widetilde{\operatorname{diff}}\left(\mathbb{T}^{n}\right)^{*}$ and $\tilde{a} \in \widetilde{\operatorname{diff}}$. $\left(\mathbb{T}^{n}\right)$. We took above; by definition [29,42]; that a loop vector field $\operatorname{Vect}_{ \pm}\left(\mathbb{T}^{n}\right) \widetilde{\operatorname{Vect}_{ \pm}}\left(\mathbb{T}^{n}\right)$ $=\Gamma\left(\tilde{T}\left(\mathbb{T}^{n}\right)\right)$ and a loop differential 1 -form $\tilde{l} \in \tilde{\Lambda}^{1}\left(\mathbb{T}^{n}\right)$ are presented in the form

$$
\begin{aligned}
& \tilde{a}=\sum_{j=1}^{n} a^{(j)}(x ; \lambda) \frac{\partial}{\partial x_{j}}:=<a(x ; \lambda), \frac{\partial}{\partial x}>, \\
& \tilde{l}=\sum_{j=1}^{n} l_{j}(x ; \lambda) d x_{j}:=<l(x ; \lambda), d x>,
\end{aligned}
$$

introduced for brevity the gradient operator $\frac{\partial}{\partial x}:=\left(\frac{\partial}{\partial x_{1}}, \frac{\partial}{\partial x_{2}}, \ldots, \frac{\partial}{\partial x_{n}}\right)^{\top}$ in the Euclidean space $\mathbb{E}^{n}$ and chose the metric $(\cdot, \cdot)_{H^{0}}$ on the space $C^{\infty}\left(\mathbb{T}^{n} ; \mathbb{R}^{n}\right) \subset H^{0}\left(\mathbb{T}^{n} ; \mathbb{R}^{n}\right)$ as

$$
(l(x ; \lambda), a(x ; \lambda))_{H^{0}}:=\sum_{j=1}^{n} \int_{\mathbb{T}^{n}} d x\left(l_{j}(x ; \lambda) a^{(j)}(x ; \lambda)\right) .
$$

The Lie commutator of vector fields $\tilde{a}, \tilde{b} \in \tilde{\mathcal{G}}$ is calculated the standard way and equals

$$
\begin{aligned}
& \tilde{a}, \tilde{b}]=\tilde{a} \tilde{b}-\tilde{b} \tilde{a}=\left\langle\left\langle a(x ; \lambda), \frac{\partial}{\partial x}\right\rangle b(x ; \lambda), \frac{\partial}{\partial x}\right\rangle- \\
& -\left\langle\left\langle b(x ; \lambda), \frac{\partial}{\partial x}\right\rangle a(x ; \lambda), \frac{\partial}{\partial x}\right\rangle .
\end{aligned}
$$

The Lie algebra $\tilde{\mathcal{G}}$ is naturally split into the direct sum of two Lie subalgebras

$$
\left.\widetilde{\operatorname{diff}}\left(\mathbb{T}^{n}\right)=\widetilde{\operatorname{diff}}+\mathbb{T}^{n}\right)_{+} \oplus \widetilde{\operatorname{diff}}\left(\mathbb{T}^{n}\right),
$$

for which one can identify the following dual spaces:

$$
\widetilde{\operatorname{diff}}_{+}\left(\mathbb{T}^{n}\right)^{*} \simeq \lambda \widetilde{\operatorname{diff}_{-}}\left(\mathbb{T}^{n}\right), \quad \widetilde{\operatorname{diff}_{-}}\left(\mathbb{T}^{n}\right)^{*} \simeq \lambda \widetilde{\operatorname{diff}_{+}}\left(\mathbb{T}^{n}\right),
$$

where for any $l(\lambda) \in \widetilde{\operatorname{diff}}$ - $\left(\mathbb{T}^{n}\right)^{*}$ there holds the constraint $\tilde{l}(0)=0$.

Construct now the Lie algebra $\tilde{\mathcal{G}}:=\widetilde{\operatorname{diff}}\left(\mathbb{T}^{n}\right) \ltimes \widetilde{\operatorname{diff}}\left(\mathbb{T}^{n}\right)^{*}$ as the semidirect sum of the Lie algebra $\widetilde{\operatorname{diff}}\left(\mathbb{T}^{n}\right)$ and its dual space $\widetilde{\operatorname{diff}}\left(\mathbb{T}^{n}\right)^{*}$, whose Lie structure is given by the following expression

$$
\left.\tilde{a}_{1} \ltimes \tilde{l}_{1}, \tilde{a}_{2} \ltimes \tilde{l}_{2}\right]:=\left[\tilde{a}_{1}, \tilde{a}_{2}\right] \ltimes\left(a d_{\tilde{a}_{1}}^{*} \tilde{l}_{2}-a d_{\tilde{a}_{2}}^{*} \tilde{l}_{1}\right)
$$

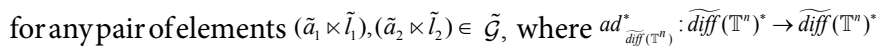
is the standard coadjoint mapping of the Lie algebra $\widetilde{\operatorname{diff}}\left(\mathbb{T}^{n}\right)$ on its adjoint space $\widetilde{\operatorname{diff}}\left(\mathbb{T}^{n}\right)^{*}$ with resspect to the pairing (169). The Lie algebra $\tilde{\mathcal{G}}$ proves to be metrizable; as it can be endowed with the nondegenerate ad-invariant bilinear symmetric product:

$$
\left(\tilde{a}_{1} \ltimes \tilde{l}_{1}, \tilde{a}_{2} \ltimes \tilde{l}_{2}\right):=\left(\tilde{l}_{2}, \tilde{a}_{1}\right)_{1}+\left(\tilde{l}_{1}, \tilde{a}_{2}\right)_{1},
$$

where $\tilde{a}_{1} \ltimes \tilde{l}_{1}, \tilde{a}_{2} \ltimes \tilde{l}_{2} \in \tilde{\mathcal{G}}$ are arbitrary elements. Owing to the holomorphic structure of the Lie algebra $\widetilde{\operatorname{diff}}\left(\mathbb{T}^{n}\right)$, the ad-invariant product (175) makes it possible to identify the Lie algebra $\tilde{\mathcal{G}}$ with its dual $\tilde{\mathcal{G}}^{*}$, that is $\tilde{\mathcal{G}}^{*} \simeq \tilde{\mathcal{G}}$. Moreover; the Lie algebra $\tilde{\mathcal{G}}$ can be naturally split $[18,19,38]$ with respect to the the pairing $(169)$ and the Lie bracket (174) into two subalgebras $\tilde{\mathcal{G}} \tilde{\mathcal{G}}_{+} \oplus \tilde{\mathcal{G}}_{-}$, where; by definition;

$$
\tilde{\mathcal{G}}_{+}:=\widetilde{\operatorname{diff}}\left(\mathbb{T}^{n}\right)_{+} \times \lambda^{-1} \widetilde{\operatorname{diff}}\left(\mathbb{T}^{n}\right)_{-}^{*}, \tilde{\mathcal{G}}_{-}:=\widetilde{\operatorname{diff}}\left(\mathbb{T}^{n}\right)_{-} \ltimes \lambda^{-1} \widetilde{\operatorname{diff}}\left(\mathbb{T}^{n}\right)_{+}^{*} .
$$

The latter allows to defiine on the Lie algebra $\tilde{\mathcal{G}}$ a new Lie bracket

$$
\left.\tilde{w}_{1}, \tilde{w}_{2}\right]_{\mathcal{R}}:=\left[\mathcal{R} \tilde{w}_{1}, \tilde{w}_{2}\right]+\left[\tilde{w}_{1}, \mathcal{R} \tilde{w}_{2}\right]
$$


Citation: Blackmore D, Hentosh EO, Prykarpatski AK (2017) The Novel Lie-Algebraic Approach to Studying Integrable Heavenly Type MultiDimensional Dynamical Systems. J Generalized Lie Theory Appl 11: 286. doi: 10.4172/1736-4337.1000286

for any elements $\tilde{w}_{1}, \tilde{w}_{2} \in \tilde{\mathcal{G}}$, where $\mathcal{R}:=\left(P_{+}-P_{-}\right) / 2$ is the standard $\mathcal{R}$-matrix homomorphism $[22,23,25]$ on $\tilde{\mathcal{G}}$ and; by definition; $P_{ \pm}: \tilde{\mathcal{G}} \rightarrow \tilde{\mathcal{G}} \subset \tilde{\mathcal{G}}$ are projectors. The construction above makes it possible to apply to the Lie algebra $\tilde{\mathcal{G}}$ the classical AKS-scheme and; respectively; to generate a wide class of completely integrable Hamiltonian systems as the commuting flows on the adjoint space $\tilde{\mathcal{G}}^{*} \simeq \tilde{\mathcal{G}}$, generated by the corresponding hierarchies of the Casimir invariants subject to the basic Lie bracket (175).

To describe this cheme in more details; we need to find the corresponding Casimir functionals $h \in I\left(\tilde{\mathcal{G}}^{*}\right)$, satisfying; by definition; the following relationship:

$$
\nabla h(\tilde{l}, \tilde{a}), \tilde{a} \ltimes \tilde{l}]=0,
$$

where; by definition; the gradient $\nabla h(\tilde{l}, \tilde{a}):=\nabla h_{\tilde{I}} \ltimes \nabla h_{\tilde{a}} \in \tilde{\mathcal{G}}$ satisfies the following from (178) differential-algebraic equations:

$$
\left.\nabla h_{\tilde{l}}, \tilde{a}\right]=0, a d_{\nabla h_{\tilde{l}}}^{*} \tilde{l}-a d_{\tilde{a}}^{*} \nabla h_{\tilde{a}}=0
$$

for arbitrarily chosen element $\tilde{a} \ltimes \tilde{l} \in \tilde{\mathcal{G}}$. The eqn. (179) can be rewritten [29] in details as

$$
\begin{aligned}
& \left\langle\nabla h_{l}, \frac{\partial}{\partial x}\right\rangle a-\left\langle a, \frac{\partial}{\partial x}\right\rangle \nabla h_{l}=0, \\
& \left\langle\frac{\partial}{\partial x}, \nabla h_{l}\right\rangle l+\left\langle l,\left(\frac{\partial}{\partial x} \nabla h_{l}\right)\right\rangle-\left\langle\frac{\partial}{\partial x}, a\right\rangle \nabla h_{a}-\left\langle\nabla h_{a},\left(\frac{\partial}{\partial x} a\right)\right\rangle=0,
\end{aligned}
$$

where we put; by definition; that

$$
\begin{aligned}
& \nabla h_{i}:=\left\langle\nabla h_{l}, \frac{\partial}{\partial x}\right\rangle, \tilde{a}:=\left\langle a, \frac{\partial}{\partial x}\right\rangle, \\
& \tilde{l}:=\langle l, d x\rangle, \nabla h_{\tilde{a}}:=\left\langle\nabla h_{a}, d x\right\rangle .
\end{aligned}
$$

The system of linear eqn. (180) for a given element $\tilde{a} \ltimes \tilde{l} \in \tilde{\mathcal{G}}$, singular as $\lambda \rightarrow \infty$, can be; in general; resolved by means of the asymptotical expressions

$$
\nabla h_{l} \sim \sum_{j \in \mathbb{Z}_{+}} \nabla h_{l}^{(j)} \lambda^{-j}, \nabla h_{a} \sim \sum_{j \in \mathbb{Z}_{+}} \nabla h_{a}^{(j)} \lambda^{-j},
$$

giving rise to an infinite hierarchy of gradients $\nabla h^{(p)}(\tilde{a}, \tilde{l}):=\lambda^{p} \nabla h(\tilde{a}, \tilde{l}) \in \tilde{\mathcal{G}}, p \in \mathbb{Z}_{+}$, for the corresponding Casimir functionals $h^{(p)} \in I\left(\tilde{\mathcal{G}}^{*}\right), p \in \mathbb{Z}_{+}$. Similarly; if a given element $\tilde{a} \ltimes \tilde{l} \in \tilde{\mathcal{G}}$ is chosen to be singular as $\lambda \rightarrow 0$, the system of linear eqn. (180) can be resolved by means of the asymptotical expressions

$$
\nabla h_{l} \sim \sum_{j \in \mathbb{Z}_{+}} \nabla h_{l}^{(j)} \lambda^{j}, \nabla h_{a} \sim \sum_{j \in \mathbb{Z}_{+}} \nabla h_{a}^{(j)} \lambda^{j},
$$

also generating an infinite hierarchy of gradients $\nabla h^{(p)}(\tilde{l}, \tilde{a})=\lambda^{-p} \nabla h(\tilde{a}, \tilde{l}) \in \tilde{\mathcal{G}}, \quad p \in \mathbb{Z}_{+}, \quad$ for the corresponding Casimir functionals $h^{(p)} \in I\left(\tilde{\mathcal{G}}^{*}\right), p \in \mathbb{Z}_{+}$.

Let us now assume that we have already found the gradients $\nabla h^{(y)}(\tilde{a}, \tilde{l}):=\lambda^{p_{y}} \nabla h^{(1)}(\tilde{a}, \tilde{l}), \quad \nabla h^{(t)}(\tilde{a}, \tilde{l}):=\lambda^{p_{y}} \nabla h^{(2)}(\tilde{a}, \tilde{l}) \in \tilde{\mathcal{G}}$, related with two Casimir invarints $h^{(1)}, h^{(2)} \in I\left(\tilde{\mathcal{G}}^{*}\right) \quad$ (not necessary different) for some integers $p_{y}, p_{t} \in \mathbb{Z}$, satisfying the determining eqn. (180). Then; owing to the classical AKS-scheme [22,23,25]; one can construct two commuting to each other flows with respect to the evolution parameters $y, t \in \mathbb{R}$ on the adjoint space $\tilde{\mathcal{G}}^{*} \simeq \tilde{\mathcal{G}}$

$$
\begin{aligned}
& \frac{\partial}{\partial y} \tilde{a}=-\left[\nabla h_{\tilde{l},+}^{(y)}, \tilde{a}\right], \frac{\partial}{\partial t} \tilde{a}=-\left[\nabla h_{\tilde{l},+}^{(t)}, \tilde{a}\right], \\
& \text { and } \\
& \frac{\partial}{\partial y} \tilde{l}=a d_{\nabla h_{l,+}^{(y)}}^{*} \tilde{l}-a d_{\tilde{a}}^{*}\left(\nabla h_{\tilde{a}_{+}}^{(y)}\right), \frac{\partial}{\partial t} \tilde{l}=a d_{\nabla h_{\tilde{l},+}^{(t)}}^{*} \tilde{l}-a d_{\tilde{a}}^{*}\left(\nabla h_{\tilde{a}_{+}}^{(t)}\right),
\end{aligned}
$$

where; we have denoted by

$$
\left(\nabla h_{l_{,+}}^{(y)} \ltimes \nabla h_{\tilde{a}_{+}}^{(y)}\right):=P_{+} \nabla h^{(y)}(\tilde{a}, \tilde{l}) \in \tilde{\mathcal{G}}_{+},\left(\nabla h_{l_{+}}^{(t)} \ltimes \nabla h_{\hat{a}_{+}}^{(t)}\right):=P_{+} \nabla h^{(t)}(\tilde{a}, \tilde{l}) \in \tilde{\mathcal{G}}_{+}
$$

the projections on positive degree parts of the corresponding asymptotic expansions (181)- (183). The flows (184) and (185) are; by construction; Hamiltonian; as they result from the expressions

$$
\frac{\partial}{\partial y}(\tilde{a} \ltimes \tilde{l})=\left\{h^{(y)}, \tilde{a} \ltimes \tilde{l}\right\}_{\mathcal{R}}, \frac{\partial}{\partial t}(\tilde{a} \ltimes \tilde{l})=\left\{h^{(t)}, \tilde{a} \ltimes \tilde{l}\right\}_{\mathcal{R}}
$$

for a chosen element $\tilde{a} \ltimes \tilde{l} \in \tilde{\mathcal{G}}^{*}$, stemming from the $\mathcal{R}$-deformed LiePoisson $[22,23,25,48]$ bracket

$$
\{h, f\}_{\mathcal{R}}:=\int_{\mathbb{S}^{1}} d z\left(\tilde{a} \ltimes \tilde{l},[\nabla h(\tilde{l}, \tilde{a}), \nabla f(\tilde{l}, \tilde{a})]_{\mathcal{R}}\right)
$$

on the space $\tilde{\mathcal{G}}^{*}$, defined for any smooth functionals $h, f \in D\left(\tilde{\mathcal{G}}^{*}\right)$. From the reasonings above one can derive by means of easy yet cumbersome calculations the following important proposition.

Proposition 5.3: The Hamiltonian flows (186) generate the separately commuting evolution flows (184) and (185); giving rise to the following unique Lax type compatibility condition:

$$
\left.\nabla h_{l,+}^{(y)}, \nabla h_{i,+}^{(t)}\right]-\frac{\partial}{\partial t} \nabla h_{l,+}^{(y)}+\frac{\partial}{\partial y} \nabla h_{l,+}^{(t)}=0,
$$

being equivalent to some system of nonlinear heavenly type equations in partial derivatives.

The presented above construction of Hamiltonian flows on the adjoint space $\tilde{\mathcal{G}}^{*}$ still allows the next important generalization. Namely; let us endow the point product $\breve{\mathcal{G}}:=\prod_{z \in \mathbb{S}^{1}} \tilde{\mathcal{G}}$ of loop Lie algebra $\tilde{\mathcal{G}}$ with the central extension generated by a two-cocycle $\omega_{2}: \breve{\mathcal{G}} \times \breve{\mathcal{G}} \rightarrow \mathbb{C}$, where

$$
\omega_{2}\left(\tilde{a}_{1} \ltimes \tilde{l}_{1}, \tilde{a}_{2} \ltimes \tilde{l}_{2}\right):=\int_{\mathbb{S} 1} d z\left[\left(l_{1}, \partial \tilde{a}_{2} / \partial z\right)_{1}-\left(l_{2}, \partial \tilde{a}_{1} / \partial z\right)_{1}\right]
$$

for any pair of elements $\tilde{a}_{1} \ltimes \tilde{l}_{1}, \tilde{a}_{2} \ltimes \tilde{l}_{2} \in \breve{\mathcal{G}}$. The resulting $\mathcal{R}$-deformed Lie-Poisson bracket (187) for any smooth functionals $h, f \in D\left(\breve{\mathcal{G}}^{*}\right)$ on the adjoint space $\breve{\mathcal{G}}^{*}$ becomes equal to

$$
\begin{aligned}
& \{h, f\}_{\mathcal{R}}:=\int_{\mathbb{S}^{1}} d z\left(\tilde{a} \ltimes \tilde{l},[\nabla h(\tilde{l}, \tilde{a}), \nabla f(\tilde{l}, \tilde{a})]_{\mathcal{R}}\right)+ \\
& +\omega_{2}(\mathcal{R} \nabla h(\tilde{l}, \tilde{a}), \nabla f(\tilde{l}, \tilde{a}))+\omega_{2}(\nabla h(\tilde{l}, \tilde{a}), \mathcal{R} \nabla f(\tilde{l}, \tilde{a})) .
\end{aligned}
$$

The corresponding Casimir functionals $h^{(p)} \in I\left(\breve{\mathcal{G}}^{*}\right), p \in \mathbb{Z}_{+}$, are defined with respect to the standard Lie-Poisson bracket as

$$
\left\{h^{(p)}, f\right\}:=\int_{\mathbb{S}^{1}} d z\left(\tilde{a} \ltimes \tilde{l},\left[\nabla h^{(p)}(\tilde{l}, \tilde{a}), \nabla f(\tilde{a}, \tilde{l})\right]\right)+\omega_{2}\left(\nabla h^{(p)}(\tilde{a}, \tilde{l}), \nabla f(\tilde{a}, \tilde{l})\right)=0
$$

for all smoth functionals $f \in D\left(\breve{\mathcal{G}}^{*}\right)$. Based on the equality (191) one easily finds that the gradients $\nabla h^{(p)} \in \breve{\mathcal{G}}$ of the Casimir functionals $h^{(p)} \in I\left(\breve{\mathcal{G}}^{*}\right), p \in \mathbb{Z}_{+}$, satisfy the following equations:

$$
-\left[\nabla h_{\tilde{l}}, \tilde{a}\right]+\frac{\partial}{\partial z} \nabla h_{\tilde{l}}=0, a d_{\nabla h_{\tilde{l}}}^{*} \tilde{l}-a d_{\tilde{a}}^{*} \nabla h_{\tilde{a}}-\frac{\partial}{\partial z} \nabla h_{\tilde{a}}=0
$$

for a chosen element $\tilde{a} \ltimes \tilde{l} \in \breve{\mathcal{G}}^{*}$. Making use of the suitable Casimir functionals $h^{(y)}, h^{(t)} \in I\left(\breve{\mathcal{G}}^{*}\right)$, one can construct from (190) the following commuting Hamiltonian flows on the adjoint space $\breve{\mathcal{G}}^{*}$ :

$$
\frac{\partial}{\partial y}(\tilde{a} \ltimes \tilde{l})=\left\{h^{(y)}, \tilde{a} \ltimes \tilde{l}\right\}_{\mathcal{R}}, \frac{\partial}{\partial t}(\tilde{a} \ltimes \tilde{l})=\left\{h^{(t)}, \tilde{a} \ltimes \tilde{l}\right\}_{\mathcal{R}},
$$

which are equivalent to the evolution equations

$\frac{\partial}{\partial y} \tilde{a}=-\left[\nabla h_{i,+}^{(y)}, \tilde{a}\right]+\frac{\partial}{\partial z} \nabla h_{i,+}^{(y)}, \frac{\partial}{\partial t} \tilde{a}=-\left[\nabla h_{i,+}^{(t)}, \tilde{a}\right]+\frac{\partial}{\partial z} \nabla h_{i,+}^{(t)}$

and

$\frac{\partial}{\partial y} \tilde{l}=a d_{\nabla h_{l,+}^{(y)}}^{*} \tilde{l}-a d_{\tilde{a}}^{*}\left(\nabla h_{\tilde{a}_{+}}^{(y)}\right)-\frac{\partial}{\partial z} \nabla h_{\tilde{a}_{+}}^{(y)}$, 
Citation: Blackmore D, Hentosh EO, Prykarpatski AK (2017) The Novel Lie-Algebraic Approach to Studying Integrable Heavenly Type MultiDimensional Dynamical Systems. J Generalized Lie Theory Appl 11: 286. doi: 10.4172/1736-4337.1000286

Page 14 of 19

$\frac{\partial}{\partial t} \tilde{l}=a d_{\nabla h_{++}^{(t)}}^{*} \tilde{l}-a d_{\tilde{a}}^{*}\left(\nabla h_{\tilde{a}_{+}}^{(t)}\right)-\frac{\partial}{\partial z} \nabla h_{\tilde{a}_{+}}^{(t)}$.

As a consequence of the obtained above results one can formulate a next proposition.

Proposition 5.4: The Hamiltonian flows (193) on the adjoint space $\breve{\mathcal{G}}^{*}$ generate the separately commuting evolution flows (194) and (195); giving rise to the following unique Lax type compatibility condition:

$$
\left.\nabla h_{l,+}^{(y)}, \nabla h_{i,+}^{(t)}\right]-\frac{\partial}{\partial t} \nabla h_{l,+}^{(y)}+\frac{\partial}{\partial y} \nabla h_{l,+}^{(t)}=0
$$

being equivalent to some system of nonlinear heavenly type equations in partial derivatives. Moreover; the system of evolution flows (194) and (195) can be considered as the compatibility condition for the following set of linear vector equations

$$
\frac{\partial \psi}{\partial y}+\nabla h_{i,+}^{(y)} \psi=0, \frac{\partial \psi}{\partial z}+\tilde{a} \psi=0, \frac{\partial \psi}{\partial t}+\nabla h_{i,+}^{(t)} \psi=0
$$

for all $\left.(y, t ; \lambda, z, x) \in \mathbb{R}^{2} \times\left(\mathbb{C} \times \mathbb{S}^{1}\right) \times \mathbb{T}^{n}\right)$ and a function $\psi \in C^{2}\left(\mathbb{R}^{2} \times \mathbb{C} \times\left(\mathbb{S}^{1} \times \mathbb{T}^{n}\right) ; \mathbb{C} \not\right.$.

The following examples demonstrate the analytical applicability of the devised above Lie-algebraic scheme for construction a wide class of nonlinear multidimensional heavenly type integrable Hamiltonian systems on functional spaces.

\section{Example: Einstein-Weyl metric equation}

Define $\overline{\mathcal{G}}^{*}=\operatorname{diff}_{h o l}\left(\mathbb{T}^{1} \times \mathbb{C}\right)$ and take the seed element

$$
\bar{l}=\left(u_{x} \lambda-2 u_{x} v_{x}-u_{y}\right) d x+\left(\lambda^{2}-v_{x} \lambda+v_{y}+v_{x}^{2}\right) d \lambda,
$$

which generates with respect to the metric (149) (as before for $q=0$ ) the gradient of the Casimir invariants $h^{(y)}, h^{(t)} \in I\left(\overline{\mathcal{G}}^{*}\right)$ in the form

$$
\begin{aligned}
& \nabla h^{(t)}(l) \sim \lambda^{2}(0,1)^{\top}+\left(-u_{x}, v_{x}\right)^{\top} \lambda+\left(u_{y}, u-v_{y}\right)^{\top}+O\left(\lambda^{-1}\right), \\
& \nabla h^{(y)}(l) \sim \lambda(0,1)^{\top}+\left(-u_{x}, v_{x}\right)^{\top}+\left(u_{y},-v_{y}\right)^{\top} \lambda^{-1}+O\left(\lambda^{-2}\right)
\end{aligned}
$$

as $|\lambda| \rightarrow \infty$ at $p_{t}=2, p_{y}=1$. For the gradients of the Casimir functions $h^{(t)}, h^{(y)} \in I\left(\mathcal{G}^{*}\right)$, determined by (158) one can easily obtain the corresponding Hamiltonian vector field generators

$$
\begin{aligned}
& \nabla h^{(t)}(\bar{l})_{+}=\left\langle\nabla h^{(t)}(l)_{+}, \frac{\partial}{\partial \mathrm{x}}\right\rangle=\left(\lambda^{2}+\lambda v_{x}+u-v_{y}\right) \frac{\partial}{\partial x}+\left(-\lambda u_{x}+u_{y}\right) \frac{\partial}{\partial \lambda}, \\
& \nabla h^{(y)}(\bar{l})_{+}=\left\langle\nabla h^{(y)}(l)_{+}, \frac{\partial}{\partial \mathrm{x}}\right\rangle=\left(\lambda+v_{x}\right) \frac{\partial}{\partial x}-u_{x} \frac{\partial}{\partial \lambda},
\end{aligned}
$$

satisfying the compatibility condition (161); which is equivalent to the set of equations

$$
\left\{\begin{array}{l}
u_{x t}+u_{y y}+\left(u u_{x}\right)_{x}+v_{x} u_{x y}-v_{y} u_{x x}=0, \\
v_{x t}+v_{y y}+u v_{x x}+v_{x} v_{x y}-v_{y} v_{x x}=0,
\end{array}\right.
$$

describing general integrable Einstein-Weyl metric equations [56].

As is well known [17]; the invariant reduction of (200) at $v=0$ gives rise to the famous dispersionless Kadomtsev-Petviashvili equation

$$
\left(u_{t}+u u_{x}\right)_{x}+u_{y y}=0,
$$

for which the reduced vector field representation (162) follows from (199) and is given by the vector fields

$$
\begin{aligned}
& \nabla h^{(t)}(\bar{l})_{+}=\left(\lambda^{2}+u\right) \frac{\partial}{\partial x}+\left(-\lambda u_{x}+u_{y}\right) \frac{\partial}{\partial \lambda}, \\
& \nabla h^{(y)}(\bar{l})_{+}=\lambda \frac{\partial}{\partial x}-u_{x} \frac{\partial}{\partial \lambda},
\end{aligned}
$$

satisfying the compatibility condition (161); equivalent to the eqn. (201). In particular; one derives from (162) and (202) the vector field compatibility relationships

$$
\begin{gathered}
\frac{\partial \psi}{\partial t}+\left(\lambda^{2}+u\right) \frac{\partial \psi}{\partial x}+\left(-\lambda u_{x}+u_{y}\right) \frac{\partial \psi}{\partial \lambda}=0 \\
\frac{\partial \psi}{\partial y}+\lambda \frac{\partial \psi}{\partial x}-u_{x} \frac{\partial \psi}{\partial \lambda}=0,
\end{gathered}
$$

satisfied for $\psi \in C^{2}\left(\mathbb{R}^{2} \times \mathbb{T}^{1} \times \mathbb{C} ; \mathbb{C}\right)$ and any $y, t \in \mathbb{R},\left(x, \lambda \not \in \mathbb{T}^{1} \times \mathbb{C}\right.$.

\section{The modified Einstein-Weyl metric equation}

This equation system is

$$
\begin{aligned}
& u_{x t}=u_{y y}+u_{x} u_{y}+u_{x}^{2} w_{x}+u u_{x y}+u_{x y} w_{x}+u_{x x} a, \\
& w_{x t}=u w_{x y}+u_{y} w_{x}+w_{x} w_{x y}+a w_{x x}-a_{y},
\end{aligned}
$$

where $a_{x}:=u_{x} w_{x}-w_{x y}$, and was recently derived [37]; In this case we take also $\overline{\mathcal{G}}^{*}=\operatorname{diff}_{h l}\left(\mathbb{T}^{1} \times \mathbb{C}\right)$, yet for a seed element $\bar{l} \in \overline{\mathcal{G}}$ we choose the form

$$
\begin{aligned}
& \bar{l}=\left[\lambda^{2} u_{x}+\left(2 u_{x} w_{x}+u_{y}+3 u u_{x}\right) \lambda+2 u_{x} \partial_{x}^{-1} u_{x} w_{x}+2 u_{x} \partial_{x}^{-1} u_{y}+\right. \\
& \left.+3 u_{x} w_{x}{ }^{2}+2 u_{y} w_{x}+6 u u_{x} w_{x}+2 u u_{y}+3 u^{2} u_{x}-2 a u_{x}\right] d x+ \\
& +\left[\lambda^{2}+\left(w_{x}+3 u\right) \lambda+2 \partial_{x}^{-1} u_{x} w_{x}+2 \partial_{x}^{-1} u_{y}+w_{x}^{2}+3 u w_{x}+3 u^{2}-a\right] d \lambda,
\end{aligned}
$$

which with respect to the metric (149) (as before for $q=0$ ) generates two Casimir invariants $h^{(y)}, h^{(t)} \in I\left(\overline{\mathcal{G}}^{*}\right)$, whose gradients; as follows from (158); equal to

$$
\begin{aligned}
& \nabla h^{(t)}(l) \sim \lambda^{2}\left[\left(u_{x},-1\right)^{\top}+\left(u u_{x}+u_{y},-u+w_{x}\right)^{\top} \lambda^{-1}+\right. \\
& \left.+\left(0, u w_{x}-a\right)^{\top} \lambda^{-2}\right]+O\left(\lambda^{-1}\right), \\
& \left.\nabla h^{(y)}(l) \sim \lambda\left(u_{x},-1\right)^{\top}+\left(0, w_{x}\right)^{\top} \lambda^{-1}\right]+O\left(\lambda^{-1}\right),
\end{aligned}
$$

as $|\lambda| \rightarrow \infty$ at $p_{y}=1, p_{t}=2$. The suitable positive projections

$$
\begin{aligned}
& \nabla h^{(y)}(l)_{+}:=\left(u_{x} \lambda,-\lambda+w_{x}\right)^{\top}, \\
& \nabla h^{(y)}(l)_{+}:=\left(u_{x} \lambda^{2}+\left(u u_{x}+u_{y}\right) \lambda,-\lambda^{2}+\left(w_{x}-u\right) \lambda+u w_{x}-a\right)^{\top} .
\end{aligned}
$$

of the gradients (206) generate the Hamiltonian flows (163); giving rise to the compatible Lax-Sato vector field system

$$
\begin{aligned}
& \frac{\partial \psi}{\partial y}+\left(-\lambda+w_{x}\right) \frac{\partial \psi}{\partial x}+u_{x} \lambda \frac{\partial \psi}{\partial \lambda}=0, \\
& \frac{\partial \psi}{\partial t}+\left(-\lambda^{2}+\left(w_{x}-u\right) \lambda+u w_{x}-a\right) \frac{\partial \psi}{\partial x}+\left(u_{x} \lambda^{2}+\left(u u_{x}+u_{y}\right) \lambda\right) \frac{\partial \psi}{\partial \lambda}=0,
\end{aligned}
$$

satisfied for $\psi \in C^{2}\left(\mathbb{R}^{2} \times \mathbb{T}^{1} \times \mathbb{C} ; \mathbb{C}\right)$ and any $y, t \in \mathbb{R},\left(x, \lambda \not \in \mathbb{T}^{1} \times \mathbb{C}\right.$.

\section{Example: The Dunajski heavenly equations}

This equation; suggested [48]; generalizes the corresponding antiself-dual vacuum Einstein equation; which is related to the Pleban'ski metric and the celebrated Plebanski [16] second heavenly eqn. (116). To study the integrability of the Dunajski equations

$$
\begin{aligned}
& u_{x_{1} t}+u_{y x_{2}}+u_{x_{1} x_{1}} u_{x_{2} x_{2}}-u_{x_{1} x_{2}}^{2}-v=0, \\
& v_{x_{1} t}+v_{x_{2} y}+u_{x_{1} x_{1}} v_{x_{2} x_{2}}-2 u_{x_{1} x_{2}} v_{x_{1} x_{2}}=0,
\end{aligned}
$$

where $\quad(u, v) \in C^{\infty}\left(\mathbb{R}^{2} \times \mathbb{T}^{2} ; \mathbb{R}^{2}\right), \quad\left(y, t ; x_{1}, x_{2}\right) \in \mathbb{R}^{2} \times \mathbb{T}^{2}, \quad$ we define $\overline{\mathcal{G}}^{*}:=\operatorname{diff}_{h o l}^{*}\left(\mathbb{C} \times \mathbb{T}^{n}\right)$ and take the following as a seed element $\bar{l} \in \overline{\mathcal{G}}^{*}$

$$
\bar{l}=\left(\lambda+v_{x_{1}}-u_{x_{1} x_{1}}+u_{x_{1} x_{2}}\right) d x_{1}+\left(\lambda+v_{x_{2}}+u_{x_{2} x_{2}}-u_{x_{1} x_{2}}\right) d x_{2}+\left(\lambda-x_{1}-x_{2}\right) d \lambda .
$$

With respect to the metric (149) (as before for $q=0$ ), the gradients of two functionally independent Casimir invariants $h^{\left(p_{y}\right)}, h^{\left(p_{t}\right)} \in I\left(\overline{\mathcal{G}}^{*}\right)$ can 
Citation: Blackmore D, Hentosh EO, Prykarpatski AK (2017) The Novel Lie-Algebraic Approach to Studying Integrable Heavenly Type MultiDimensional Dynamical Systems. J Generalized Lie Theory Appl 11: 286. doi: 10.4172/1736-4337.1000286

Page 15 of 19

be obtained as $|\lambda| \rightarrow \infty$ in the asymptotic form as

$$
\begin{aligned}
& \nabla h^{\left(p_{y}\right)}(l) \sim \lambda(0,1,0)^{\top}+\left(-v_{x_{1}},-u_{x_{1} x_{2}}, u_{x_{1} x_{1}}\right)^{\top}+O\left(\lambda^{-1}\right), \\
& \nabla h^{\left(p_{t}\right)}(l) \sim \lambda(0,0,-1)^{\top}+\left(v_{x_{2}}, u_{x_{2} x_{2}},-u_{x_{1} x_{2}}\right)^{\top}+O\left(\lambda^{-1}\right)
\end{aligned}
$$

at $p_{t}=1=p_{y}$. Upon calculating the Hamiltonian vector field generators

$$
\begin{aligned}
& \nabla h^{(y)}(l)_{+}:=\nabla h^{\left(p_{y}\right)}(l)_{+}=\left(-v_{x_{1}}, \lambda-u_{x_{1} x_{2}}, u_{x_{1} x_{1}}\right)^{\top}, \\
& \nabla h^{(t)}(\bar{l})_{+}=<\nabla h_{+}^{(t)}, \frac{\partial}{\partial \mathrm{x}}>=u_{x_{2} x_{2}} \frac{\partial}{\partial x_{1}}-\left(\lambda+u_{x_{1} x_{2}}\right) \frac{\partial}{\partial x_{2}}+v_{x_{2}} \frac{\partial}{\partial \lambda},
\end{aligned}
$$

following from the Casimir functions gradients (211); one easily obtains the following vector fields

$$
\begin{aligned}
& \nabla h^{(t)}(\bar{l})_{+}=<\nabla h_{+}^{(t)}, \frac{\partial}{\partial \mathrm{x}}>=u_{x_{2} x_{2}} \frac{\partial}{\partial x_{1}}-\left(\lambda+u_{x_{1} x_{2}}\right) \frac{\partial}{\partial x_{2}}+v_{x_{2}} \frac{\partial}{\partial \lambda}, \\
& \nabla h^{(y)}(\bar{l})_{+}=<\nabla h_{+}^{(y)}, \frac{\partial}{\partial \mathrm{x}}>=\left(\lambda-u_{x_{1} x_{2}}\right) \frac{\partial}{\partial x_{1}}+u_{x_{1} x_{1}} \frac{\partial}{\partial x_{2}}-v_{x_{1}} \frac{\partial}{\partial \lambda},
\end{aligned}
$$

satisfying the Lax-Sato compatibility condition (161)

$$
\begin{aligned}
& \frac{\partial \psi}{\partial t}+u_{x_{2} x_{2}} \frac{\partial \psi}{\partial x_{1}}-\left(\lambda+u_{x_{1} x_{2}}\right) \frac{\partial \psi}{\partial x_{2}}+v_{x_{2}} \frac{\partial \psi}{\partial \lambda}=0, \\
& \frac{\partial \psi}{\partial y}+\left(\lambda-u_{x_{1} x_{2}}\right) \frac{\partial \psi}{\partial x_{1}}+u_{x_{1} x_{1}} \frac{\partial \psi}{\partial x_{2}}-v_{x_{1}} \frac{\partial \psi}{\partial \lambda}=0,
\end{aligned}
$$

equivalent to the the Dunajski [56] eqn. (209) and satisfied for $\psi \in C^{2}\left(\mathbb{R}^{2} \times \mathbb{C} \times \mathbb{T}^{2} ; \mathbb{C}\right)$, any $\left(y, t ; x_{1}, x_{2}\right) \in \mathbb{R}^{2} \times \mathbb{T}^{2}$ and all $\lambda \in \mathbb{C}$. As was mentioned [56]; the Dunajski eqn. (209) generalize both the dispersionless Kadomtsev-Petviashvili and Pleban'ski second heavenly equations; and is also a Lax-Sato integrable quasi-Hamiltonian system.

\section{A generalized Liouville type equation}

In an interesting work [34]; devoted to studying Grassmannians; closed differential forms and related $N$-dimensional integrable systems; authors have presented; in particular; a Lax-Sato type representation for the well known Liouville equation

$$
\partial^{2} \varphi / \partial y \partial t=\exp \varphi
$$

written down in the so called "laboratory" coordinates $y, t \in \mathbb{R}^{2}$ for a function $\varphi \in C^{2}\left(\mathbb{R}^{2} ; \mathbb{R}\right)$ and having different geometric interpretations. Their related result; obtained via some completely formal calculations; reads as follows: a system of the linear vector field equations

$$
\begin{aligned}
& \partial \psi / \partial y+\left(\lambda^{2}+v \lambda+1\right) \partial \psi / \partial \lambda=0, \\
& \partial \psi / \partial t-u \partial \psi / \partial \lambda=0
\end{aligned}
$$

for a function $\psi \in C 2\left(\mathbb{R}^{2} ; \mathbb{C}\right)$ is compatible for all $y, t \in \mathbb{R}^{2}$, where $u, v \in C^{2}\left(\mathbb{R}^{2 ;} \mathbb{R}\right)$ are functional coefficients and $\lambda \in \mathbb{C}$ is a complex parameter. Under the simple reduction $u=1 / 2 \exp$ the compatibility condition for (216) coincides exactly with the Liouville eqn. (215).

Being interested in the deepest Lie-algebraic and geometric nature of the Lax-Sato representation (216) for the Liouville eqn. (215); we have posed the following problem:

to find a seed element for the complex torus diffeomorphism group $\operatorname{Diff}\left(T_{\mathbb{C}}^{1}\right)$, whose specially chosen coadjoint orbits generate the compatible system of linear vector field eqn. (216)?

As a first step for solving this problem one needs to consider the corresponding Lie algebra $\overline{\mathcal{G}}:=\operatorname{diff}\left(\mathbb{T}_{\mathbb{C}}^{1}\right)$ and its decomposition into the direct sum of subalgebras

$$
\overline{\mathcal{G}}=\overline{\mathcal{G}_{+}} \oplus \overline{\mathcal{G}}
$$

of Laurent series with positive as $z \rightarrow 0$ and strongly negative as $z \rightarrow \infty$ degrees; respectively. Then; owing to thew classical Adler-CostantSymes theory $[23,25,26,57,58]$; for any element $\bar{l} \in \overline{\mathcal{G}}^{*} \simeq \Lambda^{1}\left(\mathbb{T}_{\mathbb{C}}^{1}\right)$ the following formally constructed flows

$$
d \bar{l} / d y=-a d_{\nabla h^{(y)}(\bar{l})_{+}}^{*} \bar{l}, d \bar{l} / d t=-a d_{\nabla h^{(t)}(\bar{l})_{+}}^{*} \bar{l}
$$

along the evolution parameters $y, t \in \mathbb{R}^{2}$ are always compatible; if $h^{(y)}$ and $h^{(t)} \in I\left(\overline{\mathcal{G}}^{*}\right)$ are arbitrarily chosen functionally independent Casimir functionals on $\overline{\mathcal{G}}^{*}$, and $\nabla h^{(y)}(\bar{l})_{+}, \nabla h^{(t)}(\bar{l})_{+}$are their gradients; suitably projected on the subalgebra $\overline{\mathcal{G}_{+}}$. Keeping in mind the mentioned above result; consider the Casimir functional $h^{(y)}$ on $\overline{\mathcal{G}}^{*}$, whose gradient $\nabla h^{(y)}(\bar{l}):=\nabla h^{(y)}(l) \partial / \partial z$ as $z \rightarrow \infty$ is taken; for simplicity;

$$
\nabla h^{(y)}(\bar{l})=\left(w_{2} z^{2}+w_{1} z+w_{0}+w_{-1} z^{-1}\right) \partial / \partial z \in \overline{\mathcal{G}},
$$

giving rise to the gradient projection $\nabla h^{(y)}(\bar{l})_{+}=\left(w_{2} z^{2}+w_{1} z+w_{0}\right) \partial / \partial z \in \overline{\mathcal{G}}_{+}$, where $\quad z \in \mathbb{T}_{\mathbb{C}}^{1}, \quad z \rightarrow \infty, \quad$ is a complex torus parameter and $w_{j} \in C^{2}\left(\mathbb{R}^{2} ; \mathbb{R}\right), j=\overline{-1,2}$, are some functional parameters. As the root element $\bar{l}=l(y, t ; z) d z$ satisfies; by definition; the differential equation

$$
\frac{d}{d z}\left[l(y, t ; z)\left(\nabla h^{(y)}(l)\right)^{2}\right]=0,
$$

we obtain from (220) and (219) that the element

$$
l(y, t ; z)=\frac{c(y, t)^{2}}{\left(\nabla h^{(y)}(l)\right)^{2}}=\left(v_{2} z^{2}+v_{1} z+v_{0}+v_{-1} z^{-1}\right)^{-2},
$$

where $\quad c \in C^{2}\left(\mathbb{R}^{2} ; \mathbb{R}\right) \quad$ is an arbitrary function and $v_{j}:=w_{j} / c \in C^{2}\left(\mathbb{R}^{2} ; \mathbb{R}\right), j=\overline{-1,2}$. If to put for brevity that $v_{2}:=1$, the element (221) becomes

$$
l(y, t ; z)=\left(z^{2}+v_{1} z+v_{0}+v_{-1} z^{-1}\right)^{-2}
$$

Observe now that the relationship (220) makes it possible to formulate the following lemma.

Lemma 5.5: The set $I\left(\tilde{\mathcal{G}}^{*}\right)$ of the functionally independent Casimir invariants is one-dimensional.

As a consequence of the Lemma above we state that in the case of the element $\bar{l}=l d z \in \overline{\mathcal{G}}^{*}$, generated by the expression (221); there exists the only flow on $\overline{\mathcal{G}}^{*}$ from (218) with respect to the evolution variable $y \in \mathbb{R}:$

$$
d l / d y=\nabla h^{(y)}(l)_{+}^{-1} \frac{\partial}{\partial z}\left[l(y, t ; z) \nabla h^{(y)}(l)_{+}\right]^{2} .
$$

Concerning the flow from (218) with respect to the evolution variable $t \in \mathbb{R}$ one can take the constant functional $h^{(t)}:=$ const $\in I\left(\overline{\mathcal{G}}^{*}\right), \nabla h^{(t)}(l)=0$, and construct the trivial flow on $\overline{\mathcal{G}}^{*}$ as

$$
d l / d t=\nabla h^{(t)}(l)_{+} \frac{\partial l}{\partial z}+2 l \frac{\partial}{\partial z}\left(\nabla h^{(t)}(l)_{+}\right)=0
$$

What is now important to observe that the compatibility condition of these two flows for all $y, t \in \mathbb{R}$ is equivalent to the following system of two a priori compatible linear vector field equations

$$
\begin{aligned}
& \frac{\partial \psi}{\partial y}+\nabla h^{(y)}(l)_{+} \frac{\partial \psi}{\partial z}=0, \quad \frac{\partial \psi}{\partial t}+\nabla h^{(t)}(l)_{+} \frac{\partial \psi}{\partial z}=0, \\
& \text { or } \\
& \frac{\partial \psi}{\partial y}+\left(z^{2}+v_{1} z+v_{0}\right) \frac{\partial \psi}{\partial z}=0, \quad \frac{\partial \psi}{\partial t}+0 \frac{\partial \psi}{\partial z}=0
\end{aligned}
$$

for a smooth function $\psi \in C^{2}\left(\mathbb{R}^{2} ; \mathbb{C}\right)$, meaning; in particular; that the complex parameter $z \in \mathbb{T}_{\mathbb{C}}^{1}$ is constant with respect to the evolution 
Citation: Blackmore D, Hentosh EO, Prykarpatski AK (2017) The Novel Lie-Algebraic Approach to Studying Integrable Heavenly Type MultiDimensional Dynamical Systems. J Generalized Lie Theory Appl 11: 286. doi: 10.4172/1736-4337.1000286

parameter $t \in \mathbb{R}$. The linear eqn. (226) are; evidently; equivalent to the a priori compatible system of vector fields

$$
d z / d y=\nabla h^{(y)}(l)_{+}=z^{2}+v_{1} z+v_{0}, \quad d z / d t=\nabla h^{(y)}(l)_{+}=0
$$

on the complex torus $\mathbb{T}_{\mathbb{C}}^{1}$, which can be rewritten subject to the following diffeomorphic mapping $\mathbb{T}_{\mathbb{C}}^{1} \ni z \rightarrow z-\alpha(t, y):=\lambda \in \mathbb{T}_{\mathbb{C}}^{1}$ generated by an arbitrary smooth function $\alpha \in C^{3}\left(\mathbb{R}^{2} ; \mathbb{R}\right)$ :

$$
d \lambda / d y=\lambda^{2}+\lambda\left(2 \alpha+v_{1}\right)+\left(\alpha^{2}+\alpha v_{1}+v_{0}-\partial \alpha / \partial y\right), d \lambda / d t=-\partial \alpha / \partial t .
$$

This system is; evidently; also compatible for all $y, t \in \mathbb{R}$ and can be expressed as

$$
d \lambda / d y=\lambda^{2}+\lambda v+w, d \lambda / d t=-u,
$$

where we put; by definition;

$$
2 \alpha+v_{1}:=v, \alpha^{2}+\alpha v_{1}+v_{0}-\partial \alpha / \partial y:=w, \partial \alpha / \partial t:=u .
$$

Moreover; the a priori compatible system of linear vector field eqn. (226) can be suitably rewritten as

$$
\frac{\partial \psi}{\partial y}+\left(\lambda^{2}+v \lambda+w\right) \frac{\partial \psi}{\partial \lambda}=0, \quad \frac{\partial \psi}{\partial y}-u \frac{\partial \psi}{\partial \lambda}=0
$$

for the corresponding function $\psi \in C^{2}\left(\mathbb{R}^{2} ; \mathbb{C}\right)$, giving rise to the following system of heavenly type nonlinear equations:

$$
v_{t}-2 u=0, u_{y}-u v+w_{t}=0 .
$$

The latter can be; in particular; parametrized by means of the substitution $u:=1 / 2 \exp$ as follows:

$$
\varphi_{y t}=\exp \varphi-\left(2 w_{t} \exp (-\varphi)\right)_{t} .
$$
The next reductions $w:=1$ or $w:=-\frac{1}{2} \exp \varphi$ give rise to the well
known Liouville equations

$$
\varphi_{y t}=\exp \varphi, \varphi_{y t}-\varphi_{t t}=\exp \varphi,
$$

respectively; which; as is well known; possess [57] standard Lax type iso-spectral representations. As a result of the reasonings above one formulate as the next proposition.

Proposition 5.6: The system (232) of heavenly type nonlinear equations possesses the Lax-Sato type compatible vector field representation (231); whose Lie-algebraic structure is governed by the classical Adler-Costant-Symes theory; as it was recently developed $[28,29]$.

Concerning the starting root element $\bar{l}=l(y, t ; \lambda) d \lambda \in \overline{\mathcal{G}}^{*}$ we can take into account the relationships (230) and find from (222) that

$$
l(y, t ; \lambda)=\left(\lambda^{2}+\lambda v+w+v_{-1}(\lambda+\alpha)^{-1}\right)^{-2},
$$

where the coefficient $v_{-1} \in C^{2}\left(\mathbb{R}^{2} ; \mathbb{R}\right)$ and mapping $\alpha \in C^{3}\left(\mathbb{R}^{2} ; \mathbb{R}\right)$ are arbitrary functional parameters.

Remark 5.7: The above presented analysis can be equivalently developed for the following regularized seed-element

$$
\bar{l}(y ; z)=z^{-4}\left[1-2 v z^{-1}+\left(3 v^{2}-2 w\right) z^{-2}\right] d z \in \overline{\mathcal{G}}^{*},
$$

where $z \in \mathbb{C}$ and coefficients $v, w \in C^{2}\left(\mathbb{R}^{2} ; \mathbb{R}\right)$. The gradient of the corresponding Casimir invariant $h \in I\left(\overline{\mathcal{G}}^{*}\right)$ allows the asymptotic as $|z| \rightarrow \infty$ series representation

$$
\nabla h(l) \sim z^{2}+v z+w+w_{-1} z^{-1}+w_{-2} z^{-2}+\ldots .,
$$

whose projection on the Lie subalgebra $\overline{\mathcal{G}_{+}}$gives rise to the nontrivial evolution equation

$$
\partial \bar{l} / \partial y=-a d_{\nabla h(\bar{l})_{+}}^{*} \bar{l},
$$

where; by definition; $\nabla h(\bar{l})_{+}:=\nabla h(l)_{+} \partial / \partial z$, and which generates the related vector fields

$$
d z / d y=\nabla h(l)_{+}=z^{2}+v z+w, d z / d t=0
$$

with respect to the evolution veriables $y, t \in \mathbb{R}$. Having now made the usual change of variables $z:=\lambda-\alpha(y, t)$ for some mapping $\alpha \in C^{3}\left(\mathbb{R}^{2} ; \mathbb{R}\right)$, one can easily derive from (239) the compatible system of linear eqn. (231) with the same coefficient $u=\alpha / \partial t$.

Remark 5.8: The same way as above one can describe in detail the Lie-algebraic structure for other generalized Liouville type heavenly equations; presented in the work [34] for a higher order in $\lambda \in \mathbb{T}_{\mathbb{C}}^{1}$ system of linear vector field eqn. (225).

\section{Integrability; Bi-Hamiltonian Structures and the Classical Lagrange-d'Alembert Principle}

It is evident that all evolution flows like (57) or (163) are Hamiltonian with respect to the second Lie-Poisson bracket (153) on the adjoint loop space $\tilde{\mathcal{G}}^{*}=\widetilde{\operatorname{diff}}\left(\mathbb{T}^{n}\right)$ or on the subspace $\overline{\mathcal{G}}^{*}=\operatorname{diff} f_{\text {hol }}^{*}\left(\mathbb{C} \times \mathbb{T}^{n}\right)$, respectively. Moreover; they are poly-Hamiltonian on the corresponding functional manifolds; as the related bilinear forms (37) and (149) are marked by integers $p \in \mathbb{Z}$. This leads to [25] an infinite hierarchy of compatible Poisson structures on the phase spaces; isomorphic; respectively; to the orbits of a chosen seed element $\tilde{l} \in \tilde{\mathcal{G}}^{*}$ or of a seed element $\bar{l} \in \overline{\mathcal{G}}^{*}$. Taking also into account that all these Hamiltonian flows possess an infinite hierarchy of commuting nontrivial conservation laws; one can prove their formal complete integrability under some naturally formulated constraints. The corresponding analytical expressions for the infinite hierarchy of conservation laws can be retrieved from the asymptotic expansion (63) for Casimir functional gradients by employing the well-known $[22,23,25,58]$ formal homotopy technique.

As an arbitrary heavenly equation is a Hamiltonian system with respect to both evolution parameters $t, y \in \mathbb{R}^{2}$ and $\lambda \in \mathbb{C}$, one can construct $[22,23,45,58]$ its suitable Lagrangian (or quasi-Lagrangian) representation under some natural constraints. Thus; it is possible to retrieve the corresponding Poisson structures related to both these evolution parameters $t, y \in \mathbb{R}^{2}$ and $\lambda \in \mathbb{C}$, which; as follows from the Liealgebraic analysis in Section 3; are compatible to each other. In this way; one can show that any heavenly type equation is a bi-Hamiltonian integrable system on the corresponding functional manifold. It should be mentioned here that this property was introduced by Sergyeyev in (arXiv:1501.01955); published [53]; and rediscovered and applied in detail [30] for investigating the integrability properties of the general heavenly eqn. (121); first suggested by Schief [53] and later studied by Doubrov and Ferapontov [54].

In his book "Mecanique analytique"; v.1-2; published in 1788 in Paris; J.L. Lagrange formulated one of the basic; most general; differential variational principles of classical mechanics; expressing necessary and sufficient conditions for the correspondence of the real motion of a system of material points; subjected by ideal constraints; to the applied active forces. Within the d'Alembert-Lagrange principle the positions of the system in its real motion are compared with infinitely close positions permitted by the constraints at the given moment of time.

According to the d'Alembert-Lagrange principle; during a real motion of a system of $N \mathbb{Z}_{+}$particles with massess $F^{(j)}, j=\overline{1, N}$, the sum of the elementary works performed by the given active forces 
$F^{(j)}, j=\overline{1, N}$, and by the forces of inertia for all the possible particle displacements $\delta x^{(j)} \in \mathbb{E}^{3}, j=\overline{1, N}$, is equal to or less than zero:

$$
\sum_{j=\overline{1, N}}<F^{(j)}-m_{j} \frac{d x^{(j)}}{d t}, \delta x^{(j)}>\leq 0
$$

at any moment of time $t \in \mathbb{R}$, where $\langle\cdot, \cdot>$ denotes the standard scalar product in the three-dimensional Euclidean space $\mathbb{E}^{3}$. The equality in (240) is valid for the possible reversible displacements; the symbol $\leq$ is valid for the possible irreversible displacements $\delta x^{(j)} \in \mathbb{E}^{3}, j=\overline{1, N}$. Eqn. (240) is the general equation of the dynamics of systems with ideal constraints; it comprises all the equations and laws of motion; so that one can say that all dynamics is reduced to this single general formula.

This principle; established by J.L. Lagrange by generalization of the principle of virtual displacements with the aid of the classical d'Alembert principle. For systems subject to bilateral constraints J.L. Lagrange based himself on formula (240) to deduce the general properties and laws of motion of bodies; as well as the equations of motion; which he applied to solve a number of problems in dynamics including the problems of motions of non-compressible; compressible and elastic liquids; thus combining "dynamics and hydrodynamics as branches of the same principle and as conclusions drawn from a single general formula".

As it was first demonstrated in the work [28]; in the last case of generalized reversible motions of a compressible elastic liquid; located in a one-connected open domain $\Omega \mathbb{R}^{\mathrm{n}}$ with the smooth boundary $\partial \Omega t$, $t \in \mathbb{R}$, in space $\mathbb{R}^{n}, n \in \mathbb{Z}_{+}$, the expression (240) can be rewritten as

$$
\delta W(t):=\int_{\Omega_{t}}<l(x(t) ; \lambda), \delta x(t)>d^{n} x(t)=0
$$

for all $t \in \mathbb{R}$, where $l(x(t) ; \lambda) \in \tilde{T}^{*}\left(\mathbb{R}^{n}\right)$ is the corresponding virtual vector "reaction force"; exerted by the ambient medium on the liquid and called a seed element; which is here assumed to depend meromorphically on a constant complex parameter $\lambda \in \mathbb{C}$. If now to suppose that the evolution of liquid points $x(t) \in \Omega_{t}$ is determined for any parameters $\lambda \neq \mu \in \mathbb{C}$ by the generating gradient type vector field

$$
\frac{d x(t)}{d t}=\frac{\mu}{\mu-\lambda} \nabla h(l(\mu))(t ; x(t))
$$

and the Cauchy data

$$
\left.x(t)\right|_{t=0}=x^{(0)} \in \Omega_{0}
$$

for an arbitrarily chosen open one-connected domain $\Omega_{0} \subset \mathbb{T}^{n}$ with the smooth boundary $\partial \Omega_{0} \subset \mathbb{R}^{n}$ and a smooth functional $h: \tilde{T}^{*}\left(\mathbb{R}^{n}\right) \rightarrow \mathbb{R}$, the Lagrange-d'Alembert principle says: the infinitesimal virtual work (241) equals zero for all moments of time; that is $\delta W(t)=0=\delta W(0)$ for all $t \mathbb{R}$. To check that it is really zero; let us calculate the temporal derivative of the expression (241):

$$
\begin{gathered}
\frac{d}{d t} \delta W(t)=\frac{d}{d t} \int_{\Omega_{t}}<l(x(t) ; \lambda), \delta x(t)>d^{n} x(t)= \\
=\frac{d}{d t} \int_{\Omega_{0}}<l(x(t) ; \lambda), \delta x(t)>\left|\frac{\partial(x(t)}{\partial x_{0}}\right| d^{n} x^{(0)}=\int_{\Omega_{0}} \frac{d}{d t}\left(<l(x(t) ; \lambda), \delta x(t)>\left|\frac{\partial(x(t)}{\partial x_{0}}\right|\right) d^{n} x^{(0)}= \\
=\int_{\Omega_{0}}\left[\frac{d}{d t}<l(x(t) ; \lambda), \delta x(t)>+<l(x(t) ; \lambda), \delta x(t)>\operatorname{div} \tilde{K}(\mu)\right]\left|\frac{\partial(x(t)}{\partial x_{0}}\right| d^{n} x^{(0)}= \\
=\int_{\Omega_{t}}\left[\frac{d}{d t}<l(x(t) ; \lambda), \delta x(t)>+<l(x(t) ; \lambda), \delta x(t)>\operatorname{div} \tilde{K}(\mu)\right] d^{n} x(t)=0,
\end{gathered}
$$

if the condition

$$
\frac{d}{d t}<l(x(t) ; \lambda), \delta x(t)>+<l(x(t) ; \lambda), \delta x(t)>\operatorname{div} \tilde{K}(\mu ; \lambda)=0
$$

holds for all $t \in \mathbb{R}$, where

$$
\tilde{K}(\mu ; \lambda):=\frac{\mu}{\mu-\lambda} \nabla h(\tilde{l}(\mu))=\frac{\mu}{\mu-\lambda}<\nabla h(l(\mu)), \frac{d}{d x}>
$$

is a vector field on $\mathbb{R}^{n}$, corresponding to the evolution eqn. (242). Taking into account that the full temporal derivative $d / d t:=\partial / \partial t+L_{\tilde{K}(\mu ; \lambda)}$, where $L_{\tilde{K}(\mu ; \lambda)}=i_{\tilde{K}(\mu ; \lambda)} d+d i_{\tilde{K}(\mu ; \lambda)}$ denotes the well known $[23,44,58]$ Cartan expression for the Lie derivation along the vector field (245); can be represented as $\mu, \lambda \rightarrow \infty,|\lambda / \mu|<1$ in the asymptotic form

$$
\frac{d}{d t} \sim \sum_{j \in \mathbb{Z}_{+}} \mu^{-j} \frac{\partial}{\partial t_{j}}+\sum_{j \in \mathbb{Z}_{+}} \mu^{-j} L_{\tilde{K}_{j}(\lambda},
$$

the equality (244) can be equivalently rewritten as an infinite hierarchy of the following evolution equations

$$
\partial \tilde{l}(\lambda) / \partial t_{j}:=-a d_{\tilde{K}_{j}(\lambda)_{+}}^{*} \tilde{l}(\lambda)
$$

for every $j \in \mathbb{Z}_{+}$on the space of differential 1 -forms $\tilde{\Lambda}^{1}\left(\mathbb{R}^{n}\right) \simeq \tilde{\mathcal{G}}^{*}$, where $l(\lambda):=<l(x ; \lambda), d x>\in \Lambda(\quad) \quad, \tilde{\mathcal{G}}: \widetilde{\operatorname{diff}}\left(\mathbb{R}^{n}\right)$ is the Lie algebra of the corresponding loop diffeomorphism group $\widetilde{\operatorname{Diff}}\left(\mathbb{R}^{n}\right)$. As from (245) one easily finds that

$$
\tilde{K}_{j}(\lambda)=\nabla h^{(j)}(\tilde{l})
$$

for $\lambda \in \mathbb{C}$ and any $j \in \mathbb{Z}_{+}$, the evolution eqn. (247) transform equivalently into

$$
\partial \tilde{l}(\lambda) / \partial t_{j}:=-a d_{\nabla h^{(j)}(\tilde{l})_{+}}^{*} \tilde{l}(\lambda),
$$

allowing to formulate the following important Adler-Kostant-Symes type $[22-25,27,54]$ proposition.

Proposition 6.1: The evolution eqn. (249) are completely integrable commuting to each other Hamiltonian flows on the adjoint loop space $\tilde{\mathcal{G}}^{*}$ for a seed element $\tilde{l}(\lambda) \in \tilde{\mathcal{G}}^{*}$, generated by Casimir functionals $h^{(j)} \in I\left(\tilde{\mathcal{G}}^{*}\right)$, naturally determined by conditions $a d_{\nabla h}^{*(j)}(\tilde{l}) \vec{l}(\lambda)=0$, $j \in \mathbb{Z}_{+}$, with respect to the modified Lie-Poisson bracket on the adjoint space $\tilde{\mathcal{G}}^{*}$

$$
\{(\tilde{l}, \tilde{X}),(\tilde{l}, \tilde{Y})\}:=\left(\tilde{l},[\tilde{X}, \tilde{Y}]_{\mathcal{R}}\right),
$$

defined for any $\tilde{X}, \tilde{Y} \in \tilde{\mathcal{G}}$ by means of the canonical $\mathcal{R}$-structure on the loop Lie algebra $\tilde{\mathcal{G}}$ :

$$
\tilde{X}, \tilde{Y}]_{\mathcal{R}}:=\left[\tilde{X}_{+}, \tilde{Y}_{+}\right]-\left[\tilde{X}_{-}, \tilde{Y}_{-}\right],
$$

where " $\tilde{Z}_{ \pm}$" means the positive $(+) /(-)$-negative part of a loop Lie algebra element $\tilde{Z} \in \tilde{\mathcal{G}}$ subject to the loop parameter $\lambda \in \mathbb{C}$.

If; for instance; to consider the first two flows from (249) in the form

$$
\begin{aligned}
& \partial \tilde{l}(\lambda) / \partial t_{1}:=\partial \tilde{l}(\lambda) / \partial y=-a d_{\nabla h}^{*}(y)_{(\tilde{l})+} \\
& \partial \tilde{l}(\lambda), \\
& \partial \tilde{l}(\lambda) / \partial t_{2}:=\partial \tilde{l}(\lambda) / \partial t=-a d_{\nabla h^{(t)}(\tilde{l})_{+}}^{*} \tilde{l}(\lambda),
\end{aligned}
$$

which are; by construction; commuting to each other; from their compatibility condition ensues some system of nonlinear equations in partial derivatives on the coefficients of the seed element $\tilde{l}(\lambda) \in \tilde{\mathcal{G}}^{*}$. As the latter is; evidently; equivalent to the Lax-Sato compatibility condition of the corresponding vector fields

$$
\begin{aligned}
& \nabla h^{(y)}(\tilde{l})_{+}:=<\nabla h^{(1)}(l)_{+}, \partial / \partial x>, \nabla h^{(t)}(\tilde{l})_{+}:=<\nabla h^{(2)}(l)_{+}, \partial / \partial x>\in \tilde{\mathcal{G}}: \\
& \left.\partial / \partial y+\nabla h^{(y)}(\tilde{l})_{+}, \partial / \partial t+\nabla h^{(t)}(\tilde{l})_{+}\right]=0,
\end{aligned}
$$

a resulting from (252) system of nonlinear equations in partial derivatives is often called of heavenly type and was before actively analyzed in a series of articles $[11-13,17-21,53,54]$ and recently $[13,33,34,35]$. These 
Citation: Blackmore D, Hentosh EO, Prykarpatski AK (2017) The Novel Lie-Algebraic Approach to Studying Integrable Heavenly Type MultiDimensional Dynamical Systems. J Generalized Lie Theory Appl 11: 286. doi: 10.4172/1736-4337.1000286

Page 18 of 19

works are closely related to the problem of constructing a hierarchy of commuting to each other vector fields; analytically depending on a complex parameter $\lambda \in \mathbb{C}$, which was in general form studied and completely solved by Pfeiffer in his classical work $[6,7,9]$.

\section{Acknowledgements}

The authors cordially thank Prof. M. B łaszak; Prof. J. Cies'linski and Prof. A Sym for their cooperation and useful discussions of the results in this paper during the Workshop "Nonlinearity and Geometry" ; held 20-23 January 2017 in Warsaw and during the International Conference in Functional Analysis dedicated to the $125^{\text {th }}$ anniversary of Stefan Banach held on 18-23 September; 2017 in Lviv; Ukraine. A.P. is especially indebted to Prof. M. Pavlov; Prof. W.K. Schief and Prof. Ya. G. Prytula for mentioning important references, which were very helpful when preparing the manuscript. He is also greatly indebted to Prof. V.E. Zakharov (University of Arizona; Tucson) and Prof. J. Szmigelski (University of Saskatchewan; Saskatoon) for their interest in the work and instructive discussions during the XXXV Workshop on Geometric Methods in Physics; held 26.06-2.07.2016 in Biał owieża; Poland.

\section{References}

1. Buhl MA (1928) Surles operateurs differentieles permutables ou non. Bull des Sc Math 2: 353-361.

2. Buhl MA (1928) Apercus modernes sur la theorie des groupes continue et finis Mem des Sc Math fasc XXXIII, Paris.

3. Pfeiffer MG (1928) Sur la permutation des solutions s'une equation lineaire aux derivees partielles du premier ordre. Bull des Sc Math 2: 353-361.

4. Pfeiffer MG (1930) Generalisation de la methode de Jacobi pour l'integration des systems complets des equations lineaires et homogenes. Comptes Rendues de l'Academie des Sciences de l'URSS 190: 405-409

5. Pfeiffer MG (1930) Sur la operateurs d'un systeme complet d'equations lineaires et homogenes aux derivees partielles du premier ordre d'une fonction inconnue. Comptes Rendues de l'Academie des Sciences de l'URSS 190: 909-911.

6. Pfeiffer MG (1930) La generalization de methode de Jacobi-Mayer. Comptes Rendues de l'Academie des Sciences de l'URSS 191: 1107-1109.

7. Pfeiffer MG (1928) Quelgues additions au probleme de M. Buhl, Atti de Congresso Internationale dei Matematici, Bologna, pp: 45-46

8. Pfeiffer MG (1931) La construction des operateurs d'une equation lineaire homogene aux derivees partielles premier ordre. Journal du Cycle Mathematique, Academie des Sciences d'Ukraine, Kyiv pp: 37-72.

9. Popovici C (1907) Sur les fonctions adjointes de M. Buhl. Comptes Rendus 145: 749

10. Takasaki K, Takebe T (1991) SDiff(2) Toda equation - Hierarchy, Tau function, and symmetries. Letters in Mathematical Physics 23: 205-214.

11. Takasaki K, Takebe T (1995) Integrable Hierarchies and Dispersionless Limit Reviews in Mathematical Physics 7: 743-808.

12. Bogdanov LV, Dryuma VS, Manakov SV (2007) Dunajski generalization of the second heavenly equation: dressing method and the hierarchy. J Phys A Math Theor 40: 14383-14393.

13. Sato $M$, Sato $Y$ (1982) Soliton equations as dynamical systems on infinite dimensional Grassmann manifold in Nonlinear Partial Differential Equations in Applied Science. Proceedings of the U.S.-Japan Seminar, Tokyo Lect Notes Num Anal 5: 259-271.

14. Sato M, Noumi M (1984) Soliton equations and the universal Grassmann manifolds. Sophia University Kokyuroku in Math V:18 .

15. Pleban'ski JF (1975) Some solutions of complex Einstein equations. J Math Phys 16: 2395-2402.

16. Manakov SV, Santini PM (2009) On the solutions of the second heavenly and Pavlov equations. J Phys A Math Theor 42: 11.

17. Ovsienko $\mathrm{V}$ (2008) Bi-Hamilton nature of the equation $u_{t x}=u_{x y} u_{y}-u_{y y} u_{x}$. Math-ph p: 13.

18. Ovsienko V, Roger C (2007) Looped Cotangent Virasoro Algebra and NonLinear Integrable Systems in Dimension $2+1$. Commun Math Phys 273: 357 378

19. Pavlov MV (2003) Integrable hydrodynamic chains. J Math Phys 44: 41344156
20. Schief WK (1996) Self-dual Einstein spaces via a permutability theorem for the Tzitzeica equation. Phys Lett A 223: 55-62.

21. łaszak MB (2002) Classical R-matrices on Poisson algebras and related dispersionless systems. Phys Lett A 297: 191-195.

22. Blackmore D, Prykarpatsky AK, Samoylenko VH (2012) Nonlinear dynamical systems of mathematical physics. World Scientific Publisher, NJ, USA.

23. łaszak MB, Szablikowski BM (2002) Classical R-matrix theory of dispersionless systems: II. $(2+1)$ dimension theory. J Phys A Math Gen 35: 10345

24. Takhtajan LA, Faddeev LD (1987) Hamiltonian Approach in Soliton Theory. Springer, Berlin-Heidelberg.

25. Reyman AG, Semenov-Tian-Shansky MA (2003) Integrable Systems, The Computer Research Institute Publ. Moscow-Izhvek.

26. Prykarpatsky YA, Samoilenko AM (2002) Algebraic - analytic aspects of integrable nonlinear dynamical systems and their perturbations. Kyiv Inst Mathematics Publisher V: 41

27. Hentosh OE, Prykarpatsky YA, Denis Blackmore, Prykarpatski AK (2017) Lie-algebraic structure of Lax-Sato integrable heavenly equations and the Lagrange-d'Alembert principle. Journal of Geometry and Physics 120: 208-227.

28. Prykarpatsky YA, Prykarpatski AK (2017) The integrable heavenly type equations and their Lie-algebraic structure.

29. Sheftel MB, Malykh AA, Yaz CD (2016) Recursion operators and bi-Hamiltonian structure of the general heavenly equation.

30. Kulish PP (1986) An analogue of the Korteweg-de Vries equation for the superconformal algebra. Differential geometry. Lie groups and mechanics $\mathrm{pp}$ : 142-149.

31. Mikhalev VG (1992) On the Hamiltonian formalism for Korteweg-de Vries type hierarchies. Funct Anal Appl 26: 140-142.

32. Bogdanov LV, Pavlov MV (2016) Linearly degenerate hierarchies of quasiclassical SDYM type.

33. Bogdanov LV, Konopelchenko BG (2006) On the heavenly equation and its reductions. J Phys A Math Gen 39: 11793-11802.

34. Konopelchenko BG (2013) Grassmanians $\operatorname{Gr}(N-1, N+1)$ closed differential $N-1$ forms and $\mathrm{N}$-dimensional integrable systems.

35. Ferapontov EV, Moss J (2012) Linearly degenerate PDEs and quadratic line complexes.

36. Szablikowski B (2016) Hierarchies of Manakov-Santini Type by Means of RotaBaxter and Other Identities. SIGMA 12: 22

37. Sergyeyev A, Szablikowski BM (2008) Central extensions of cotangent universal hierarchy: (2+1)-dimensional bi-Hamiltonian systems. Phys Lett A 372: 7016-7023.

38. Kruglikov B, Morozov O (2015) Integrable dispersionless PDE in 4D, their symmetry pseudogroups and deformations. Letters in Mathematical Physics 105: 1703-1723.

39. Alonso LM, Shabat AB (2004) Hydrodynamic reductions and solutions of a universal hierarchy. Theor Math Phys104: 1073-1085.

40. Dunajski M, Kryn'ski W (2014) Einstein-Weyl geometry, dispersionless Hirota equation and Veronese webs. Mathematical Proceedings of the Cambridge Philosophical Society V: 157

41. Cartan A (1971) Differential Forms. Dover Publisher, USA.

42. Coddington EA, Levinson N (1955) Theory of ordinary differential equations International series in pure and applied mathematics, McGraw-Hill.

43. Godbillon C (1969) Geometrie Differentielle et Mecanique Analytique. Hermann Publ.

44. Prykarpatsky AK, Mykytyuk IV (1998) Algebraic integrability of nonlinear dynamical systems on manifolds: classical and quantum aspects. Kluwer Academic Publishers, the Netherlands.

45. Dunajski M (2012) Anti-self-dual four-manifolds with a parallel real spinor. Proc Roy Soc A 458: 1205

46. Misiolek G (1998) A shallow water equation as a geodesic flow on the BottVirasoro group. J Geom Phys 24 : 203-208. 
Citation: Blackmore D, Hentosh EO, Prykarpatski AK (2017) The Novel Lie-Algebraic Approach to Studying Integrable Heavenly Type MultiDimensional Dynamical Systems. J Generalized Lie Theory Appl 11: 286. doi: 10.4172/1736-4337.1000286

47. Semenov-Tian-Shansky M (1983) What is a classical R-matrix? Func Anal Appl 17: $259-272$

48. Ferapontov EV, Moro A, Sokolov VV (2009) Hamiltonian systems of hydrodynamic type in 2+1 dimensions. Comm Math Phys 285: 31-65.

49. Pavlov MV (2004) Classification of integrable Egorov hydrodynamic chains Theoret and Math Phys 13: 45-58.

50. Morozov OI (2012) A two-Component generalization of teh integrable rd-Dym equation. SIGMA 8: 051-056.

51. Krichever IM (1994) The -function of the universal Whitham hierarchy, matrix models and topological field theories. Comm Pure Appl Math 47: 437-475.

52. Sergyeyev A, Morozov OI (2014) Th efour-dimensional Martinez-AlonsoShabat equation: reductions, nonlocal symmetries, and a four-dimensional integrrabale generalization of the ABC equation. Preprint submitted to JGP V: 11.
53. Schief WK (1999) Self-dual Einstein spaces and a discrete Tzitzeica equation. A permutability theorem link. In Symmetries and Integrability of Difference Equations, Clarkson P and Nijhoff F, eds, London Mathematical Society, Lecture Note Series 255, Cambridge University Press pp: 137-148.

54. Doubrov B, Ferapontov EV (2010) On the integrability of symplectic MongeAmpère equations. J Geom Phys 60:1604-1616.

55. Gelfand IM, Fuchs DB (1968) Cohomology of the Lie algebra of vector fields on the circle. Funct Anal Appl 2: 342-343.

56. Dunajski M, Mason LJ, Tod P (2001) Einstein-Weyl geometry, the dKP equation and twistor theory. J. Geom. Phys 37: 63-93.

57. Olver P (1993) Applications of Lie Groups to Differential Equations, Second Edition, Springer-Verlag, New York.

58. Arnold VI, Mathematical Methods of Classical Mechanics. Springer, NY, USA 